Supporting information (NMR spectra of isolated compounds (1-10) and Summary of the dereplicated compounds.

\title{
Antiplasmodial Oleanane Triterpenoids from Terminalia albida Root Bark
}

Mamadou A Baldé, Emmy Tuenter, Mohamed S Traoré, Laura Peeters, An Matheeussen, Paul Cos, Guy Caljon, Tom Vermeyen, Wouter Herrebout, Aliou M Balde, Luc Pieters*, and Kenn Foubert

\section{AUTHOR INFORMATION}

\section{Corresponding Author}

Luc Pieters - Natural Products \& Food Research and Analysis (NatuRA), Department of Pharmaceutical Sciences, University of Antwerp, Universiteitsplein 1, B-2610 Antwerp, Belgium; Phone: +32 326527 15. E-mail: luc.pieters@uantwerpen.be

\section{Authors}

Mamadou A Baldé - Natural Products \& Food Research and Analysis (NatuRA), Department of Pharmaceutical Sciences, University of Antwerp, Universiteitsplein 1, B2610 Antwerp, Belgium

Emmy Tuenter - Natural Products \& Food Research and Analysis (NatuRA), Department of Pharmaceutical Sciences, University of Antwerp, Universiteitsplein 1, B-2610 Antwerp, Belgium

Mohamed S Traoré - Department of Pharmacy, University Gamal Abdel Nasser of Conakry, BP 1017, Conakry, Guinea/ Research and Valorization Center on Medicinal Plants Dubreka, BP 6411, Conakry, Guinea

Laura Peeters - Natural Products \& Food Research and Analysis (NatuRA), Department of Pharmaceutical Sciences, University of Antwerp, Universiteitsplein 1, B-2610 Antwerp, Belgium

An Matheeussen - Laboratory of Microbiology, Parasitology and Hygiene (LMPH), Faculty of Pharmaceutical, Biomedical and Veterinary Sciences, University of Antwerp, Universiteitsplein 1, B-2610 Antwerp, Belgium 
Paul Cos - Laboratory of Microbiology, Parasitology and Hygiene (LMPH), Faculty of Pharmaceutical, Biomedical and Veterinary Sciences, University of Antwerp, Universiteitsplein 1, B-2610 Antwerp, Belgium

Guy Caljon - Laboratory of Microbiology, Parasitology and Hygiene (LMPH), Faculty of Pharmaceutical, Biomedical and Veterinary Sciences, University of Antwerp, Universiteitsplein 1, B-2610 Antwerp, Belgium

Tom Vermeyen - MolSpec Research Group, Department of Chemistry, University of Antwerp, Groenenborgerlaan 171, 2020 Antwerp, Belgium

Wouter Herrebout - MolSpec Research Group, Department of Chemistry, University of Antwerp, Groenenborgerlaan 171, 2020 Antwerp, Belgium

Aliou M Balde - Department of Pharmacy, University Gamal Abdel Nasser of Conakry, BP 1017, Conakry, Guinea/ Research and Valorization Center on Medicinal Plants Dubreka, BP 6411, Conakry, Guinea

Kenn Foubert - Natural Products \& Food Research and Analysis (NatuRA), Department of Pharmaceutical Sciences, University of Antwerp, Universiteitsplein 1, B-2610 Antwerp, Belgium 


\section{CONTENTS}

\section{A. FIGURES}

Figure S1. Clusters corresponding to the oleanane triterpenoid type of the butanolic fraction

Figure S2. ${ }^{1} \mathrm{H}$ NMR spectrum (pyridine- $d_{5}, 400 \mathrm{MHz}$ ) of compound (1)

Figure S3. COSY spectrum (pyridine- $d_{5}, 400 \mathrm{MHz}$ ) of compound (1)

Figure S4. HSQC spectrum of (pyridine-d5, $400 \mathrm{MHz}$ ) of compound (1)

Figure S5. HMBC spectrum of (pyridine- $d_{5}, 400 \mathrm{MHz}$ ) of compound (1)

Figure S6. HR-ESI-MS spectrum of compound (1)

Figure S7. ${ }^{1} \mathrm{H}$ NMR spectrum ${ }^{1} \mathrm{H}$ NMR spectrum (pyridine- $d_{5}, 400 \mathrm{MHz}$ ) of compound (2)

Figure S8. ${ }^{13} \mathrm{C}$ NMR spectrum (pyridine- $d_{5}, 100 \mathrm{MHz}$ ) of compound (2)

Figure S9. COSY spectrum (pyridine- $d_{5}, 400 \mathrm{MHz}$ ) of compound (2)

Figure S10. HSQC spectrum of (pyridine- $d_{5}, 400 \mathrm{MHz}$ ) of compound (2)

Figure S11. HMBC spectrum of (pyridine- $d_{5}, 400 \mathrm{MHz}$ ) of compound (2)

Figure S12. HR-ESI-MS spectrum of compound (2)

Figure S13. ${ }^{1} \mathrm{H}$ NMR spectrum (pyridine- $d_{5}, 400 \mathrm{MHz}$ ) of compound (3)

Figure S14. COSY spectrum (pyridine- $d_{5}, 400 \mathrm{MHz}$ ) of compound (3)

Figure S15. HSQC spectrum of (pyridine- $d_{5}, 400 \mathrm{MHz}$ ) of compound (3)

Figure S16. HMBC spectrum of (pyridine- $d_{5}, 400 \mathrm{MHz}$ ) of compound (3)

Figure S17. HR-ESI-MS spectrum of compound (3)

Figure S18. ${ }^{1} \mathrm{H}$ NMR spectrum (pyridine- $d_{5}, 400 \mathrm{MHz}$ ) of compound (4)

Figure S19. ${ }^{13} \mathrm{C}$ NMR spectrum (pyridine- $d_{5}, 100 \mathrm{MHz}$ ) of compound (4)

Figure S20. COSY spectrum (pyridine- $d_{5}, 400 \mathrm{MHz}$ ) of compound (4)

Figure S21. HSQC spectrum of (pyridine- $d_{5}, 400 \mathrm{MHz}$ ) of compound (4)

Figure S22. HMBC spectrum of (pyridine- $d_{5}, 400 \mathrm{MHz}$ ) of compound (4)

Figure S23. HR-ESI-MS spectrum of compound (4)

Figure S24. ${ }^{1} \mathrm{H}$ NMR spectrum (methanol- $d_{4}, 400 \mathrm{MHz}$ ) of compound (5)

Figure S25. COSY spectrum (methanol- $d_{4}, 400 \mathrm{MHz}$ ) of compound (5)

Figure S26. HSQC spectrum of (methanol- $d_{4}, 400 \mathrm{MHz}$ ) of compound (5)

Figure S27. HMBC spectrum of (methanol- $d_{4}, 400 \mathrm{MHz}$ ) of compound (5)

Figure S28. HR-ESI-MS spectrum of compound (5)

Figure S29. ${ }^{1} \mathrm{H}$ NMR spectrum (pyridine- $d_{5}, 400 \mathrm{MHz}$ ) of compound (6)

Figure S30. ${ }^{13} \mathrm{C}$ NMR spectrum (pyridine- $d_{5}, 100 \mathrm{MHz}$ ) of compound (6)

Figure S31. COSY spectrum (pyridine- $d_{5}, 400 \mathrm{MHz}$ ) of compound (6)

Figure S32. HSQC spectrum of (pyridine- $d_{5}, 400 \mathrm{MHz}$ ) of compound (6) 
Figure S33. HMBC spectrum of (pyridine- $d_{5}, 400 \mathrm{MHz}$ ) of compound (6)

Figure S34. HR-ESI-MS spectrum of compound (6)

Figure S35. ${ }^{1} \mathrm{H}$ NMR spectrum (pyridine- $d_{5}, 400 \mathrm{MHz}$ ) of compound (7)

Figure S36. ${ }^{13} \mathrm{C}$ NMR spectrum (pyridine- $d_{5}, 100 \mathrm{MHz}$ ) of compound (7)

Figure S37. COSY spectrum of (pyridine- $d_{5}, 400 \mathrm{MHz}$ ) of compound (7)

Figure S38. HSQC spectrum of (pyridine- $d_{5}, 400 \mathrm{MHz}$ ) of compound (7)

Figure S39. HMBC spectrum of (pyridine- $d_{5}, 400 \mathrm{MHz}$ ) of compound (7)

Figure S40. HR-ESI-MS spectrum of compound (7)

Figure S41. ${ }^{1} \mathrm{H}$ NMR spectrum (pyridine- $d_{5}, 400 \mathrm{MHz}$ ) of compound (8)

Figure S42. COSY spectrum (pyridine- $d_{5}, 100 \mathrm{MHz}$ ) of compound (8)

Figure S43. HSQC spectrum of (pyridine- $d_{5}, 400 \mathrm{MHz}$ ) of compound (8)

Figure S44. HMBC spectrum of (pyridine- $d_{5}, 400 \mathrm{MHz}$ ) of compound (8)

Figure S45. HR-ESI-MS spectrum of compound (8)

Figure S46. ${ }^{1} \mathrm{H}$ NMR spectrum (pyridine- $d_{5}, 400 \mathrm{MHz}$ ) of compound (9)

Figure S47. COSY spectrum (pyridine- $d_{5}, 100 \mathrm{MHz}$ ) of compound (9)

Figure S48. HSQC spectrum of (pyridine- $d_{5}, 400 \mathrm{MHz}$ ) of compound (9)

Figure S49. HMBC spectrum of (pyridine- $d_{5}, 400 \mathrm{MHz}$ ) of compound (9)

Figure S50. HR-ESI-MS spectrum of compound (9)

Figure S51. ${ }^{1} \mathrm{H}$ NMR spectrum (pyridine- $d_{5}, 400 \mathrm{MHz}$ ) of compound (10)

Figure S52. ${ }^{13} \mathrm{C}$ NMR spectrum (pyridine- $d_{5}, 100 \mathrm{MHz}$ ) of compound (10)

Figure S53. DEPT-135 NMR spectrum (pyridine- $d 5,400 \mathrm{MHz}$ ) of compound (10)

Figure S54. COSY spectrum (Pyridine- $d 5,100 \mathrm{MHz}$ ) of compound (10)

Figure S55. HSQC spectrum of (pyridine- $d 5_{5}, 400 \mathrm{MHz}$ ) of compound (10)

Figure S56. HMBC spectrum of (pyridine- $d_{5}, 400 \mathrm{MHz}$ ) of compound (10)

Figure S57. HR-ESI-MS spectrum of compound (10) 
Table S1. Summary of dereplicated compounds from the $n$-BuOH fraction of Terminalia albida.

\begin{tabular}{|c|c|c|c|c|c|c|c|c|}
\hline $\mathrm{N}^{\circ}$ & $\begin{array}{l}\mathrm{RT} \\
(\min )\end{array}$ & Compound identification & $\begin{array}{l}\text { Molecular } \\
\text { Formula }\end{array}$ & \multicolumn{2}{|c|}{ ESI negative full } & $\begin{array}{l}\text { ESI negative mode } \\
\text { MS/MS }\end{array}$ & $\Delta \mathrm{ppm}$ & MS level \\
\hline 1 & 4.98 & Inerminoside $\mathrm{A} 1^{\mathrm{c}}$ & $\mathrm{C}_{21} \mathrm{H}_{31} \mathrm{O}_{14}$ & 507.1714 & {$[\mathrm{M}-\mathrm{H}]-$} & $297.7334,161.8494$ & 0.00 & L3 \\
\hline 2 & 5.13 & Inerminoside A1 isomer ${ }^{c}$ & $\mathrm{C}_{21} \mathrm{H}_{31} \mathrm{O}_{14}$ & 507.1721 & {$[\mathrm{M}-\mathrm{H}]-$} & $476.0734,342.8264$ & 1.38 & L3 \\
\hline 3 & 5.74 & Rehmannioside $\mathrm{A}^{\mathbf{c}}$ & $\mathrm{C}_{21} \mathrm{H}_{32} \mathrm{O}_{15}$ & 523.1667 & {$[\mathrm{M}-\mathrm{H}]-$} & 361.1405 & 0.76 & L3 \\
\hline 4 & 6.54 & Dicaffeoylacteoside $^{\mathrm{b}}$ & $\mathrm{C}_{20} \mathrm{H}_{30} \mathrm{O}_{12}$ & 461.1661 & {$[\mathrm{M}-\mathrm{H}]-$} & $315,297,161,135238$ & 0.43 & L3 \\
\hline 5 & 10.51 & Ducheside $\mathrm{A}^{\mathrm{b}}$ & $\mathrm{C}_{20} \mathrm{H}_{15} \mathrm{O}_{12}$ & 447.0562 & {$[\mathrm{M}-\mathrm{H}]-$} & 300.9984 & -0.4 & L3 \\
\hline 6 & 10.69 & Ellagic acid ${ }^{\mathbf{a}}$ & $\mathrm{C}_{14} \mathrm{H}_{5} \mathrm{O}_{8}$ & 300.998 & {$[\mathrm{M}-\mathrm{H}]-$} & 283.991 & -1.32 & L1 \\
\hline 7 & 10.89 & Compound $1^{\mathrm{a}}$ & $\mathrm{C}_{37} \mathrm{H}_{57} \mathrm{O}_{14}$ & 725.3723 & {$[\mathrm{M}-\mathrm{H}+\mathrm{FA}]-$} & $\begin{array}{lll}679.3710, & 517 . & 3156, \\
455.3158 & & \\
\end{array}$ & -3.44 & L1 \\
\hline 8 & 10.94 & Compound $2^{\mathrm{a}}$ & $\mathrm{C}_{36} \mathrm{H}_{55} \mathrm{O}_{12}$ & 679.3690 & {$[\mathrm{M}-\mathrm{H}]-$} & $517.3660,455.3156$ & 0.58 & L1 \\
\hline 9 & 11.04 & Triterpenoid saponin-O-hexoside ${ }^{c}$ & $\mathrm{C}_{37} \mathrm{H}_{59} \mathrm{O}_{14}$ & 727.3911 & {$[\mathrm{M}-\mathrm{H}]-$} & 565.3389 & -0.82 & L2 \\
\hline 10 & 11.76 & Centellasaponin $\mathrm{B}^{\mathbf{d}}$ & $\mathrm{C}_{43} \mathrm{H}_{69} \mathrm{O}_{18}$ & 873.4453 & {$[\mathrm{M}-\mathrm{H}+\mathrm{FA}]-$} & $\begin{array}{l}827.4402, \quad 711.3956, \\
503.3370,485.9660\end{array}$ & -3.54 & L3 \\
\hline 11 & 12.01 & Triterpenoid saponin-O-hexoside ${ }^{c}$ & $\mathrm{C}_{37} \mathrm{H}_{59} \mathrm{O}_{14}$ & 727.3891 & {$[\mathrm{M}-\mathrm{H}]-$} & 565.3373 & -1.92 & $\mathrm{~L} 2$ \\
\hline 12 & 12.13 & Centellasaponin B isomer ${ }^{\mathbf{c}}$ & $\mathrm{C}_{43} \mathrm{H}_{69} \mathrm{O}_{18}$ & 873.4459 & {$[\mathrm{M}-\mathrm{H}+\mathrm{FA}]-$} & $\begin{array}{l}827.4435, \quad 711.3937, \\
665.3922,503.3365\end{array}$ & -2.86 & L3 \\
\hline 13 & 12.58 & Triterpenoid saponin-O-hexoside ${ }^{c}$ & $\mathrm{C}_{37} \mathrm{H}_{59} \mathrm{O}_{14}$ & 727.3925 & [M-H]- & 565.3372 & 2.74 & L2 \\
\hline 14 & 12.72 & $\begin{array}{l}3 \alpha, \quad 21 \beta, \quad 29 \text {-trihydroxy-16- } \\
\text { oxoserrat-14-en-24-oic acid }\end{array}$ & $\mathrm{C}_{30} \mathrm{H}_{45} \mathrm{O}_{6}$ & 501.3214 & {$[\mathrm{M}-\mathrm{H}]-$} & $483.3156,439.2803$ & 0.39 & L3 \\
\hline 15 & 12.73 & Componud $3^{a}$ & $\mathrm{C}_{37} \mathrm{H}_{57} \mathrm{O}_{14}$ & 725.3753 & {$[\mathrm{M}-\mathrm{H}+\mathrm{FA}]-$} & $\begin{array}{l}679.3690, \quad 517.3157, \\
499.3056,455.3156\end{array}$ & 0.68 & L1 \\
\hline 16 & 13.08 & Ellagic Acid methylester $\mathbf{b}$ & $\mathrm{C}_{15} \mathrm{H}_{7} \mathrm{O}_{8}$ & 315.0138 & [M-H]- & 299.9905 & -0.95 & L2 \\
\hline
\end{tabular}




\begin{tabular}{|c|c|c|c|c|c|c|c|c|}
\hline 17 & 13.16 & Arjunglucoside $e^{\mathbf{a}, \mathbf{c}}$ & $\mathrm{C}_{37} \mathrm{H}_{59} \mathrm{O}_{13}$ & 711.3956 & {$[\mathrm{M}-\mathrm{H}+\mathrm{FA}]-$} & $665.3917,503.3381$ & 0.00 & L1 \\
\hline 18 & 13.56 & Sericoside ${ }^{\mathbf{a}, \mathbf{c}}$ & $\mathrm{C}_{37} \mathrm{H}_{59} \mathrm{O}_{13}$ & 711.3988 & {$[\mathrm{M}-\mathrm{H}+\mathrm{FA}]-$} & $665.3896,503.3371$ & 4.4 & L1 \\
\hline 19 & 14.03 & Centellasaponin B isomer ${ }^{\mathbf{c}}$ & $\mathrm{C}_{43} \mathrm{H}_{69} \mathrm{O}_{18}$ & 873.4476 & {$[\mathrm{M}-\mathrm{H}+\mathrm{FA}]-$} & $\begin{array}{l}827.4431, \quad 711.3954, \\
665.2607,503.3378\end{array}$ & -0.91 & L3 \\
\hline 20 & 14.32 & 2,3,8-Tri-O-Methylellagic acid ${ }^{\mathbf{a}}$ & $\mathrm{C}_{17} \mathrm{H}_{11} \mathrm{O}_{8}$ & 343.0452 & {$[\mathrm{M}-\mathrm{H}]-$} & $297.9746,268.8221$ & -0.58 & L1 \\
\hline 21 & 14.75 & Quercotriterpenoside $^{\mathbf{d}}$ & $\mathrm{C}_{43} \mathrm{H}_{61} \mathrm{O}_{15}$ & 817.3991 & {$[\mathrm{M}-\mathrm{H}]-$} & $\begin{array}{l}799.3206, \quad 781.3249, \\
448.7066\end{array}$ & -2.32 & L3 \\
\hline 22 & 14.98 & Quercotriterpenoside isomer ${ }^{\mathbf{d}}$ & $\mathrm{C}_{43} \mathrm{H}_{61} \mathrm{O}_{15}$ & 817.3979 & {$[\mathrm{M}-\mathrm{H}]-$} & $\begin{array}{ll}799.3895, & 781.3054, \\
648.2248 & \end{array}$ & -3.79 & L3 \\
\hline 23 & 15.08 & Kermesic Acid ${ }^{c, d}$ & $\mathrm{C}_{16} \mathrm{H}_{9} \mathrm{O}_{8}$ & 329.0292 & {$[\mathrm{M}-\mathrm{H}]-$} & 285.003 & -1.51 & L3 \\
\hline 24 & 15.28 & Chebuloside II $^{\text {a }}$ & $\mathrm{C}_{37} \mathrm{H}_{59} \mathrm{O}_{13}$ & 711.3939 & {$[\mathrm{M}-\mathrm{H}+\mathrm{FA}]-$} & $\begin{array}{ll}665.3924, & 503.3366, \\
485.3336 & \\
\end{array}$ & -2.38 & L1 \\
\hline 25 & 15.71 & $\begin{array}{l}\text { 1-Methyl-2-carboxy-3,5,6,8- } \\
\text { tetrahydroxyanthraquinone }\end{array}$ & $\mathrm{C}_{16} \mathrm{H}_{9} \mathrm{O}_{8}$ & 329.0289 & {$[\mathrm{M}-\mathrm{H}]-$} & 312.9984 & -2.4 & L2 \\
\hline 26 & 15.92 & Galiosin $^{\mathbf{b}}$ & $\mathrm{C}_{26} \mathrm{H}_{25} \mathrm{O}_{16}$ & 593.1134 & {$[\mathrm{M}-\mathrm{H}]-$} & 549.3071 & -1.51 & L1 \\
\hline 27 & 15.97 & Compound $5^{\mathrm{a}}$ & $\mathrm{C}_{30} \mathrm{H}_{47} \mathrm{O}_{7}$ & 519.3328 & {$[\mathrm{M}-\mathrm{H}]-$} & 501.3214 & 1.15 & L1 \\
\hline 28 & 16.25 & Compound $4^{a}$ & $\mathrm{C}_{30} \mathrm{H}_{45} \mathrm{O}_{7}$ & 517.3156 & {$[\mathrm{M}-\mathrm{H}]-$} & $499.3062,455.3158$ & -1.73 & L1 \\
\hline 29 & 16.61 & Arjunglucoside II ${ }^{\mathbf{a}, \mathbf{b}}$ & $\mathrm{C}_{36} \mathrm{H}_{57} \mathrm{O}_{10}$ & 695.4003 & {$[\mathrm{M}-\mathrm{H}]-$} & 633.2308 & -1.84 & L1 \\
\hline 30 & 16.63 & Paradoxoside $\mathrm{C}^{\mathbf{d}}$ & $\mathrm{C}_{37} \mathrm{H}_{58} \mathrm{O}_{12}$ & 695.3998 & {$[\mathrm{M}-\mathrm{H}]-$} & 469.7827 & 0.4 & L2 \\
\hline 31 & 16.79 & Compound $9^{a}$ & $\mathrm{C}_{30} \mathrm{H}_{47} \mathrm{O}_{6}$ & 503.3373 & {$[\mathrm{M}-\mathrm{H}]-$} & $487.3083,469.7904$ & 0.00 & L1 \\
\hline 32 & 16.80 & Compound $6^{a}$ & $\mathrm{C}_{30} \mathrm{H}_{45} \mathrm{O}_{7}$ & 517.3170 & {$[\mathrm{M}-\mathrm{H}]-$} & $499.3062,455.3162$ & 0.96 & L1 \\
\hline 33 & 17.29 & $\begin{array}{l}2 \alpha, 3 \beta, 21 \beta, 23 \text { tetra hydroxy- } \\
\text { olean-12-en-28 oic-acid }{ }^{\mathrm{a}, \mathrm{b}}\end{array}$ & $\mathrm{C}_{30} \mathrm{H}_{47} \mathrm{O}_{6}$ & 503.3394 & {$[\mathrm{M}-\mathrm{H}]-$} & 485.3279 & 1.78 & L1 \\
\hline 34 & 17.57 & Compound $7^{\mathrm{a}}$ & $\mathrm{C}_{30} \mathrm{H}_{45} \mathrm{O}_{7}$ & 517.3187 & {$[\mathrm{M}-\mathrm{H}]-$} & $499.3072,455.3169$ & 4.25 & L1 \\
\hline 35 & 18.22 & Arjungenin ${ }^{\mathrm{a}, \mathrm{b}}$ & $\mathrm{C}_{30} \mathrm{H}_{47} \mathrm{O}_{6}$ & 503.3370 & {$[\mathrm{M}-\mathrm{H}]-$} & $489.3279,469,7824$ & 0.79 & L1 \\
\hline
\end{tabular}




\begin{tabular}{|c|c|c|c|c|c|c|c|c|}
\hline 36 & 18.80 & Coumpound $10^{\mathrm{a}}$ & $\mathrm{C}_{30} \mathrm{H}_{47} \mathrm{O}_{6}$ & 503.3370 & {$[\mathrm{M}-\mathrm{H}]-$} & 499.3057, 455.3159 & -0.59 & $\mathrm{~L} 2$ \\
\hline 37 & 18.84 & Compound $8^{a}$ & $\mathrm{C}_{30} \mathrm{H}_{43} \mathrm{O}_{6}$ & 499.3067 & {$[\mathrm{M}-\mathrm{H}]-$} & $487.3062,455.3162$ & 1.40 & L1 \\
\hline 38 & 18.93 & Terminolic acid $^{\mathbf{a}}$ & $\mathrm{C}_{30} \mathrm{H}_{47} \mathrm{O}_{6}$ & 503.3364 & {$[\mathrm{M}-\mathrm{H}]-$} & $485.3279,467.7102$ & -1.78 & L1 \\
\hline 39 & 18.96 & $\begin{array}{l}\text { Tetrahydroxyolean-12-en-28-oic } \\
\text { acid }^{\text {a }}\end{array}$ & $\mathrm{C}_{31} \mathrm{H}_{49} \mathrm{O}_{8}$ & 549.3433 & {$[\mathrm{M}-\mathrm{H}+\mathrm{FA}]-$} & $503.3377,485.3283$ & 1.09 & L1 \\
\hline 40 & 18.97 & Arjunglucoside II ${ }^{\mathbf{a}}$ & $\mathrm{C}_{36} \mathrm{H}_{57} \mathrm{O}_{10}$ & 649.3941 & {$[\mathrm{M}-\mathrm{H}]-$} & 487.3080 & 1.69 & L3 \\
\hline 41 & 19.50 & Seric acid $^{\mathbf{a}}$ & $\mathrm{C}_{30} \mathrm{H}_{47} \mathrm{O}_{6}$ & 503.3384 & {$[\mathrm{M}-\mathrm{H}]-$} & $485.3270,441.3448$ & -2.18 & L1 \\
\hline 42 & 20.86 & Galloyl arjunolic acid isomer ${ }^{\mathbf{d}}$ & $\mathrm{C}_{37} \mathrm{H}_{51} \mathrm{O}_{9}$ & 639.3523 & {$[\mathrm{M}-\mathrm{H}]-$} & 594.3665 & -1.56 & $\mathrm{~L} 2$ \\
\hline 43 & 20.89 & Arjunic acid ${ }^{a}$ & $\mathrm{C}_{30} \mathrm{H}_{47} \mathrm{O}_{5}$ & 487.3415 & {$[\mathrm{M}-\mathrm{H}]-$} & 469.3319 & -1.64 & L1 \\
\hline 44 & 21.80 & Galloyl arjunolic acid isomer ${ }^{\mathbf{d}}$ & $\mathrm{C}_{37} \mathrm{H}_{51} \mathrm{O}_{9}$ & 639.3553 & {$[\mathrm{M}-\mathrm{H}]-$} & 594.7043 & 3.12 & L2 \\
\hline 45 & 21.86 & Galloyl arjunolic acid isomer ${ }^{\mathbf{d}}$ & $\mathrm{C}_{37} \mathrm{H}_{51} \mathrm{O}_{9}$ & 639.3525 & {$[\mathrm{M}-\mathrm{H}]-$} & 594.6952 & -1.25 & $\mathrm{~L} 2$ \\
\hline
\end{tabular}

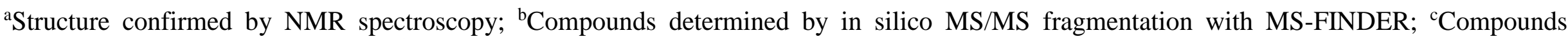
identified by molecular networking; ${ }^{\mathrm{d} C o m p o u n d s ~ t e n t a t i v e ~ i d e n t i f i e d ~ b a s e d ~ o n ~ M S, ~ M S 2 ~ e x p e r i m e n t a l ~ d a t a ~}$ 
Table S2. . ${ }^{1} \mathrm{H}$-NMR and ${ }^{13} \mathrm{C}-\mathrm{NMR}$ assignments ( $\delta_{\mathrm{c}}$ in ppm) for compounds 1,3 (100 MHz, pyridine- $\left.d 5\right)$

\begin{tabular}{|c|c|c|c|c|}
\hline \multirow[b]{2}{*}{ Position } & \multicolumn{2}{|c|}{$\mathbf{1}^{\mathbf{b}}$} & \multicolumn{2}{|c|}{$\mathbf{3}^{\mathbf{b}}$} \\
\hline & $\delta_{\mathrm{H}}(\mathrm{J}$ in $\mathrm{Hz})$ & $\delta \mathrm{C}$ & $\delta_{\mathrm{H}}(\mathrm{J}$ in $\mathrm{Hz})$ & $\delta \mathrm{C}$ \\
\hline $1 \mathrm{a} ; 1 \mathrm{~b}$ & $1.54, \mathrm{t}(11.6) ; 3.94, \mathrm{dd}(3.7,12.8)$ & $48.3, \mathrm{CH}_{2}$ & $1.26, \mathrm{~m} ; 3.72, \mathrm{dd}(3.7,11.7)$ & $48.4, \mathrm{CH}_{2}$ \\
\hline 3 & $4.23, \mathrm{~m}$ & 77.5, $\mathrm{CH}$ & $3.53, \mathrm{~d}(9.3)$ & $85.1, \mathrm{CH}$ \\
\hline 4 & & $43.6, \mathrm{C}$ & & $43.9, \mathrm{C}$ \\
\hline 5 & 1.87, br d (11.7) & $47.4, \mathrm{CH}$ & $1.06, \mathrm{~d}(11.7)$ & $55.9, \mathrm{CH}$ \\
\hline $7 \mathrm{a} ; 7 \mathrm{~b}$ & $1.43, \mathrm{~m} ; 1.83, \mathrm{~m}$ & $32.9, \mathrm{CH}_{2}$ & $1.59, \mathrm{~m} ; 1.38, \mathrm{~m}$ & $33.5, \mathrm{CH}_{2}$ \\
\hline 8 & & $46.5, \mathrm{C}$ & & $45.2, \mathrm{C}$ \\
\hline 9 & $2.90, \mathrm{~s}$ & $62.5, \mathrm{CH}$ & $2.57, \mathrm{~s}$ & $61.8, \mathrm{CH}$ \\
\hline 10 & & $38.5, \mathrm{C}$ & & $38.2, \mathrm{C}$ \\
\hline 11 & & $200.2, \mathrm{C}$ & & 199.4, C \\
\hline 12 & $6.11, \mathrm{~s}$ & $128.9, \mathrm{CH}$ & $6.36, \mathrm{~s}$ & $131.4, \mathrm{CH}$ \\
\hline 13 & & $170.6, \mathrm{C}$ & & $162.2, \mathrm{C}$ \\
\hline $15 \mathrm{a} ; 15 \mathrm{~b}$ & $1.28, \mathrm{~d}(13,7) ; 1.35^{\mathrm{a}}$ & $28.6, \mathrm{CH}_{2}$ & $1.34^{\mathrm{a}} ; 2.44$, br t $(13.4)$ & $28.1, \mathrm{CH}_{2}$ \\
\hline $16 \mathrm{a} ; 16 \mathrm{~b}$ & $2.12, \mathrm{~d}(12.5) ; 2.75, \mathrm{t}(12.5)$ & $27.4, \mathrm{CH}_{2}$ & 2.09, br d $(13.4) ; 2.20$, br t $(13.4)$ & $24.8, \mathrm{CH}_{2}$ \\
\hline 17 & & $45.7, \mathrm{C}$ & & 45.3 \\
\hline 18 & $3.57, \mathrm{~d}(5.2)$ & $45.4, \mathrm{CH}$ & $3.23, \mathrm{~d}(10.6)$ & $49.5, \mathrm{CH}$ \\
\hline 19 & $3.59, \mathrm{~d}(5.2)$ & $80.0, \mathrm{CH}$ & $3.80, \mathrm{~d}(10.6)$ & $74.5, \mathrm{CH}$ \\
\hline 20 & & $35.4, \mathrm{C}$ & & $36.1, \mathrm{C}$ \\
\hline $21 \mathrm{a} ; 21 \mathrm{~b}$ & $0.99, \mathrm{~m} ; 1.96^{\mathrm{a}}$ & $28.4, \mathrm{CH}_{2}$ & $1.63, \mathrm{~m} ; 1.28, \mathrm{~m}$ & $34.3, \mathrm{CH}_{2}$ \\
\hline $22 \mathrm{a} ; 22 \mathrm{~b}$ & $1.96^{\mathrm{a}} ;$ n.o & $31.9, \mathrm{CH}_{2}$ & $1.75, \mathrm{~m} ; 1.87, \mathrm{dd}(14.2,10.0,4.3)$ & $31.1, \mathrm{CH}_{2}$ \\
\hline $23 \mathrm{a} ; 23 \mathrm{~b}$ & $4.22, \mathrm{~m} ; 3.69, \mathrm{~d}(10.9)$ & $65.6, \mathrm{CH}_{2}$ & $1.54, \mathrm{~s} ; \mathrm{n} . \mathrm{o}$ & $23.8, \mathrm{CH}_{2}$ \\
\hline $24 \mathrm{a} ; 24 \mathrm{~b}$ & $1.06, \mathrm{~s} ; \mathrm{n} . \mathrm{o}$ & $14.6, \mathrm{CH}_{3}$ & $3.72, \mathrm{~d}(11.7) ; 4.45, \mathrm{~d}(11.7)$ & $65.4, \mathrm{CH}_{2}$ \\
\hline 25 & $1.46, \mathrm{~s}$ & $18.3, \mathrm{CH}_{3}$ & $1.37, \mathrm{~s}$ & $18.6, \mathrm{CH}_{3}$ \\
\hline 26 & $1.37, \mathrm{~s}$ & $19.4, \mathrm{CH}_{3}$ & $1.32, \mathrm{~s}$ & $19.2, \mathrm{CH}_{3}$ \\
\hline 27 & $1.67, \mathrm{~s}$ & $22.7, \mathrm{CH}_{3}$ & $1.46, \mathrm{~s}$ & $21.5, \mathrm{CH}_{3}$ \\
\hline
\end{tabular}




\begin{tabular}{cccc}
\hline 28 & & $176.6, \mathrm{C}$ & $175.3, \mathrm{C}$ \\
\hline 29 & $1.06, \mathrm{~s}$ & $28.1, \mathrm{CH}_{3}$ & $1.20, \mathrm{~s}$ \\
\hline 30 & $0.85, \mathrm{~s}$ & $24.0, \mathrm{CH}_{3}$ & $1.01, \mathrm{~s}$ \\
\hline $1^{\prime}$ & $6.35, \mathrm{~d}(8.0)$ & $95.8, \mathrm{CH}$ & $6.30, \mathrm{~d}(8.0)$ \\
\hline $2^{\prime}$ & $4.18, \mathrm{t}(8.3)$ & $73.9, \mathrm{CH}$ & $4.19, \mathrm{t}(8.3)$ \\
\hline $3^{\prime}$ & $4.27, \mathrm{t}(8.7)$ & $7.28, \mathrm{t}(8,6)$ & $7.6, \mathrm{CH}_{3}$ \\
\hline $4^{\prime}$ & $4.35^{\mathrm{a}}$ & $78.6, \mathrm{CH}$ & $7.36, \mathrm{t}(9.4)$ \\
\hline $5^{\prime}$ & $4.03, \mathrm{~m}$ & $70.8, \mathrm{CH}$ & $4.04, \mathrm{~m}$ \\
\hline $6^{\prime}$ & $4.40, \mathrm{~m}, 4.44 \mathrm{~m}$ & $79.3, \mathrm{CH}$ & $7.44, \mathrm{~m}$ \\
\hline
\end{tabular}

a Overlapping signals, byridine-d5, 
A
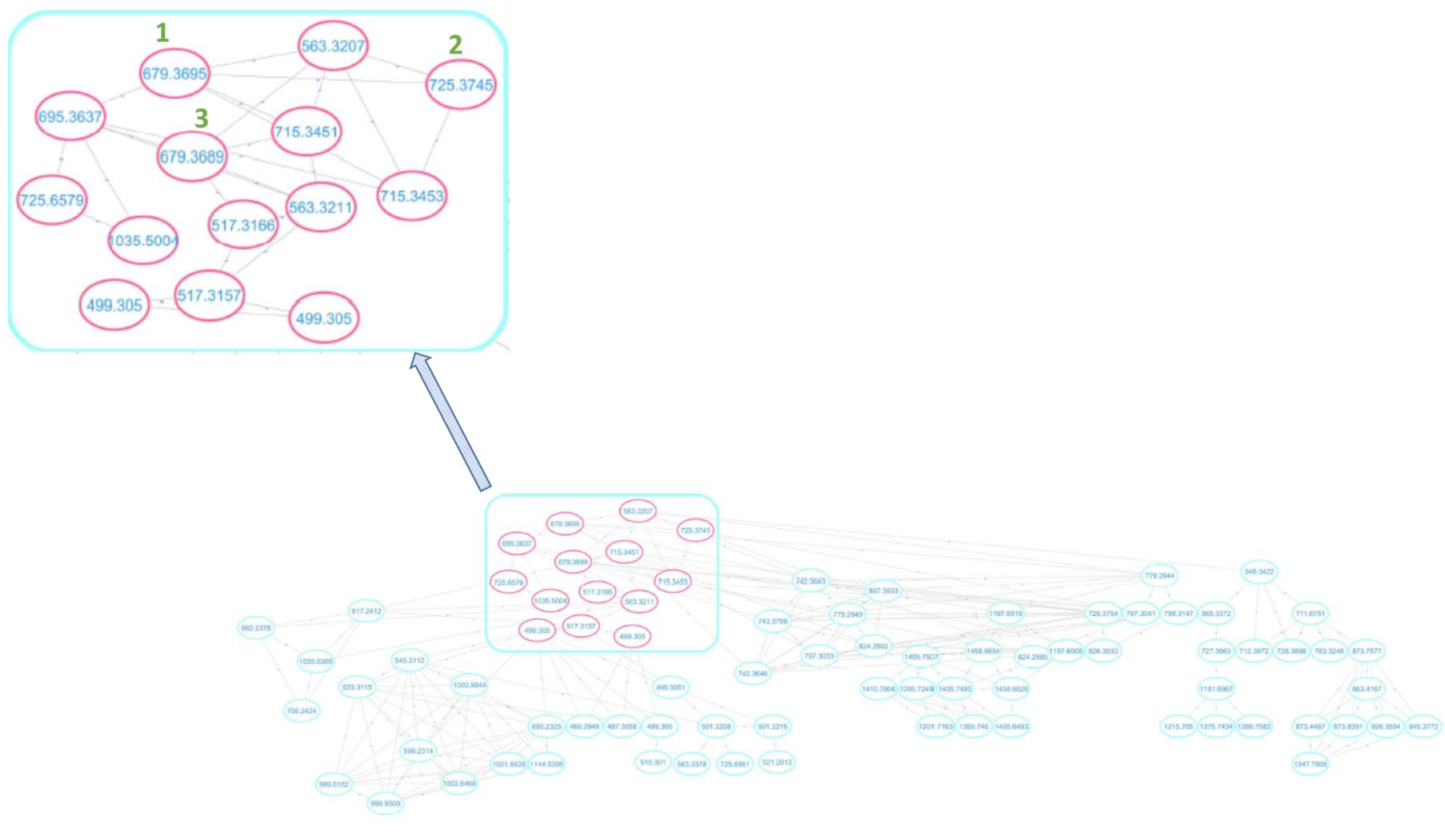


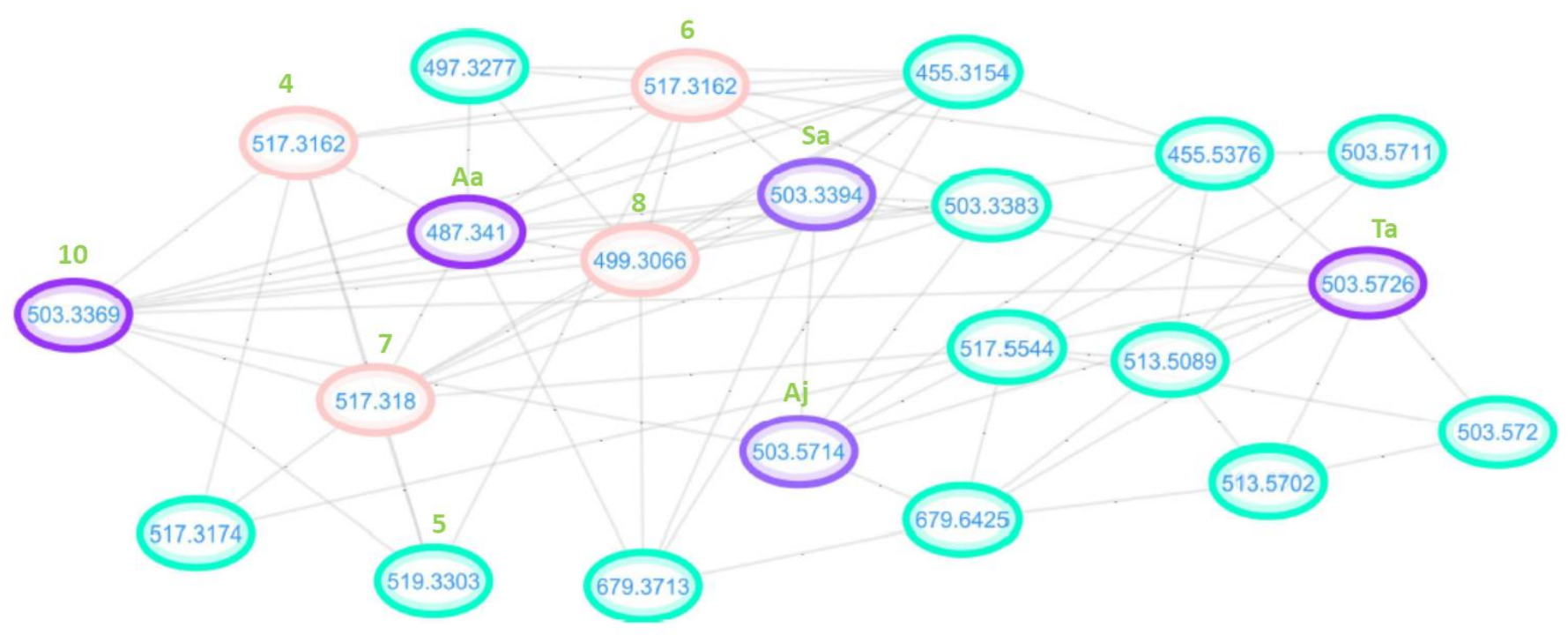

Figure S1. (A) and (B): Clusters corresponding to the oleanane triterpenoid type of the $n$-BuOH fraction with nodes labelled (compound 1 - 10) and previously isolated compounds: Ar (arjunglucoside I), Aj (arjunglucoside II), Ar (arjungenine), C (Chebuloside II), s (sericoside), sa (sericic acid). 


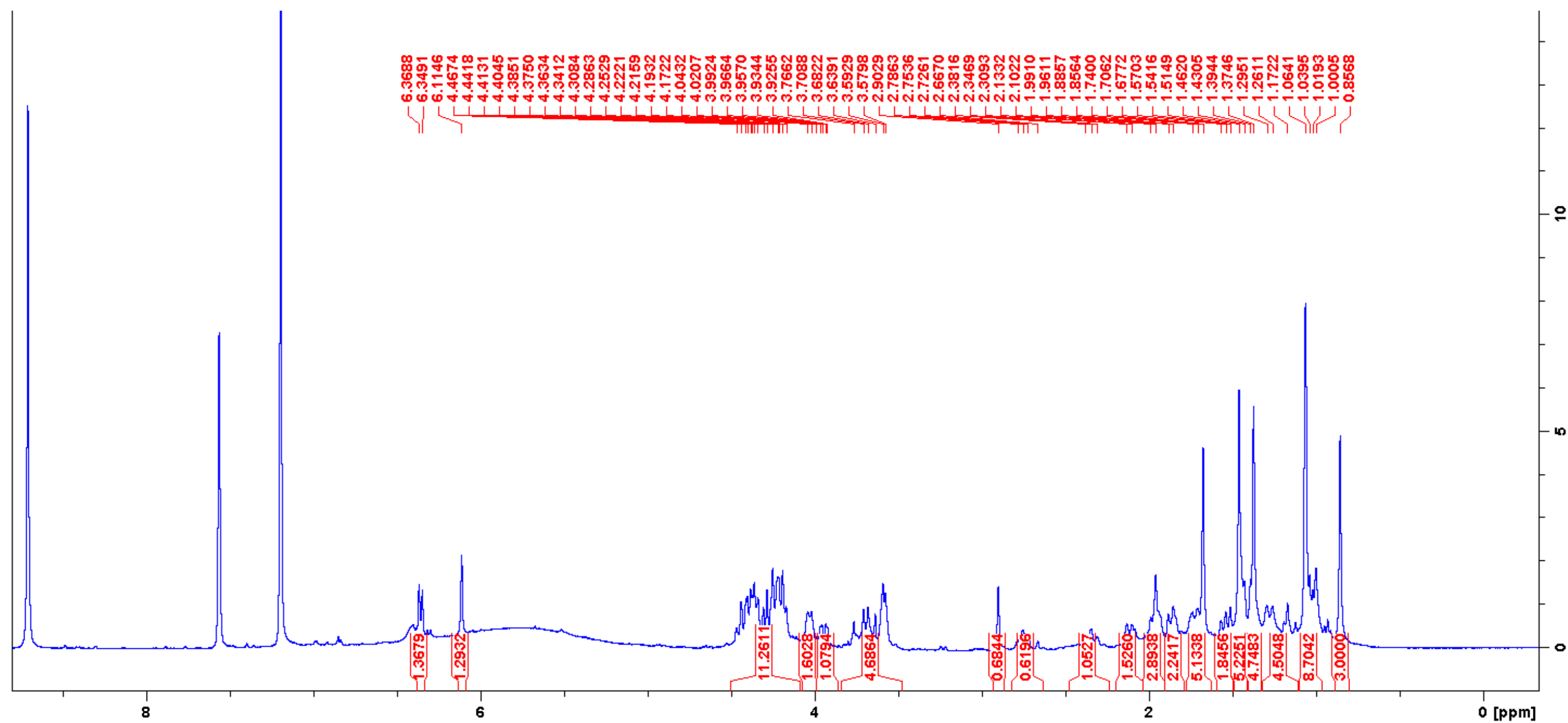

Figure S2. ${ }^{1} \mathrm{H}$ NMR spectrum (pyridine- $d_{5}, 400 \mathrm{MHz}$ ) of compound (1) 


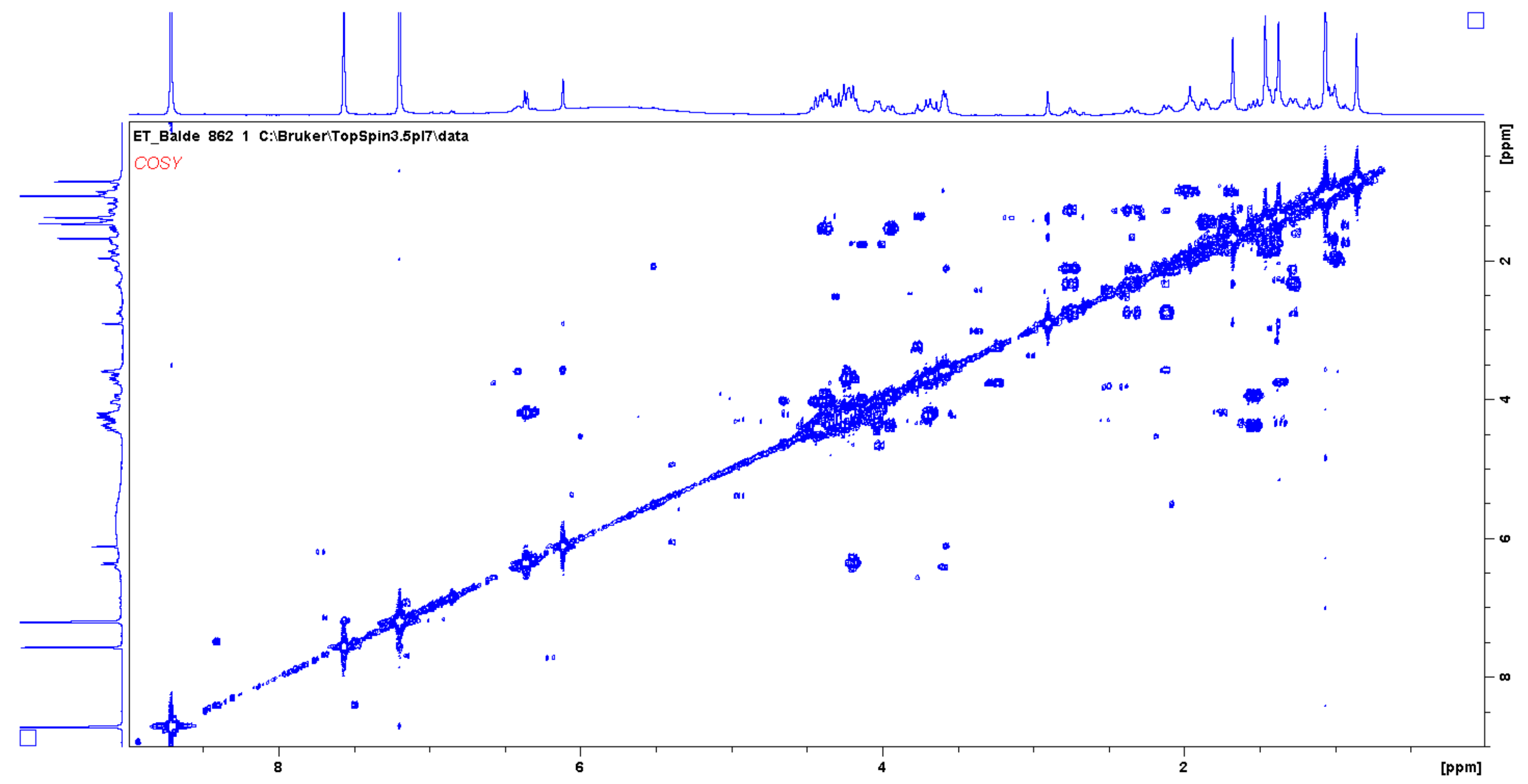

Figure S3. COSY spectrum (pyridine- $d_{5}$ ) of compound (1) 


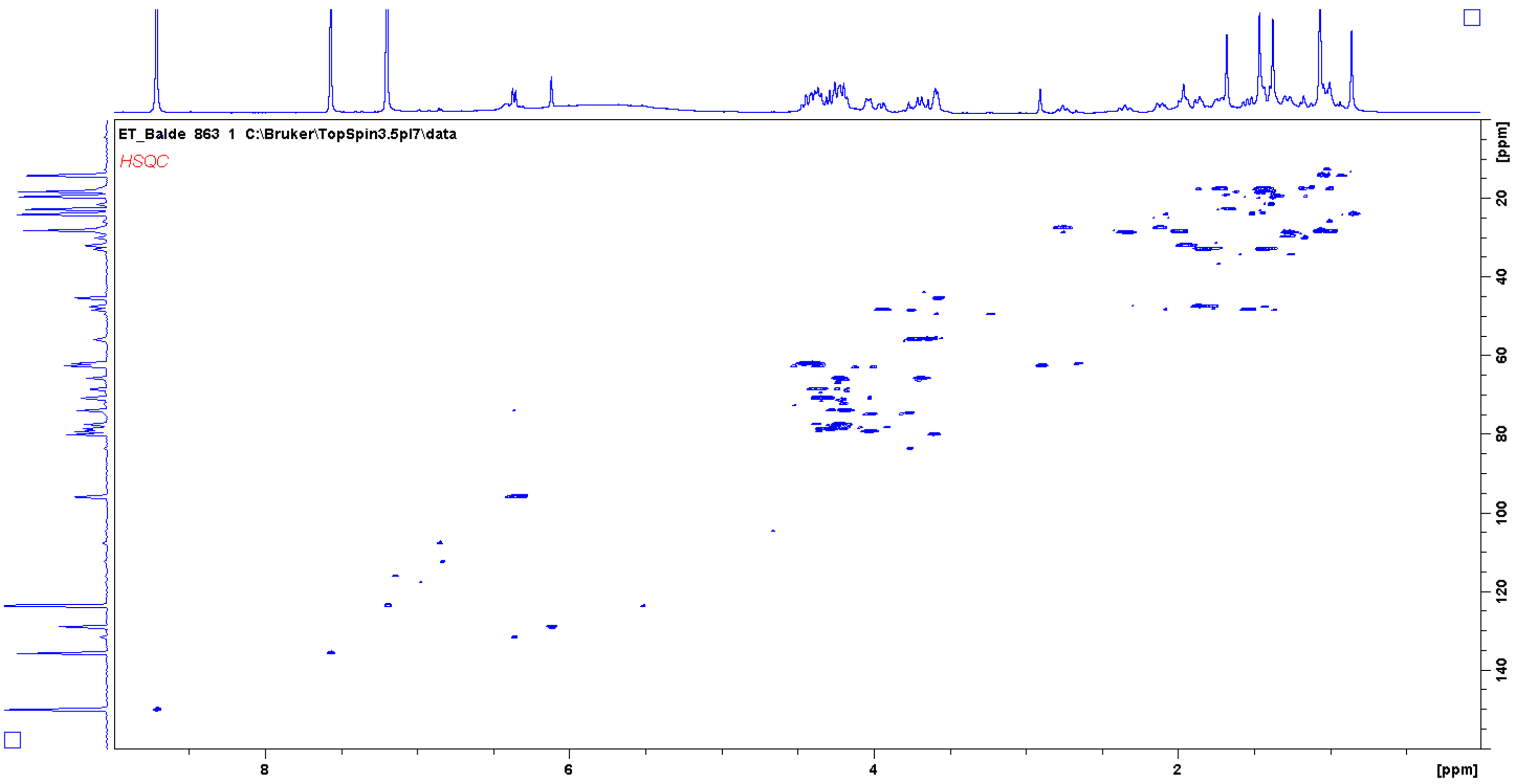

Figure S4. HSQC spectrum (pyridine- $d_{5}$ ) of compound (1) 


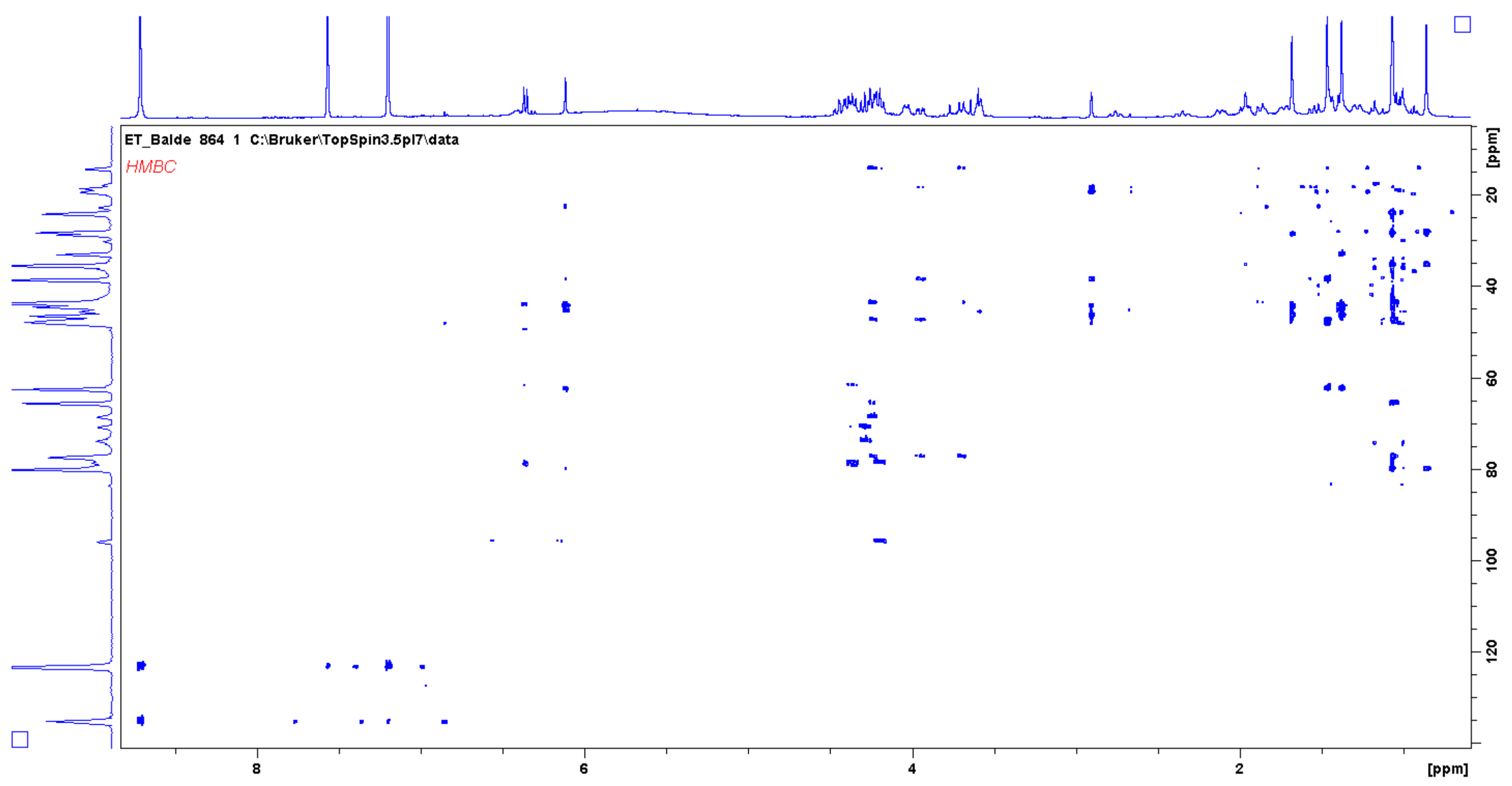

Figure S5. HMBC spectrum (pyridine- $d_{5}$ ) of compound (1) 


\section{Compound 1}

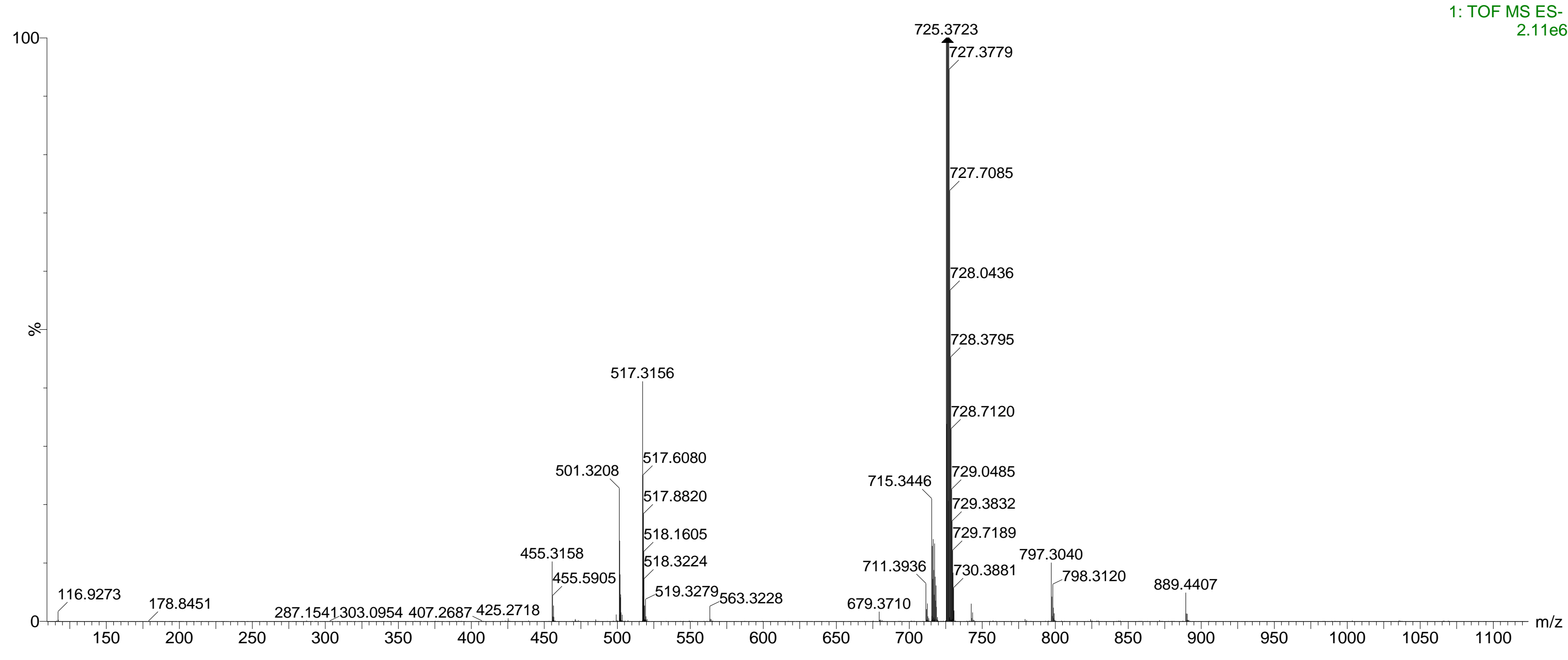

Figure S6. HR-ESI-MS spectrum of compound (1) 


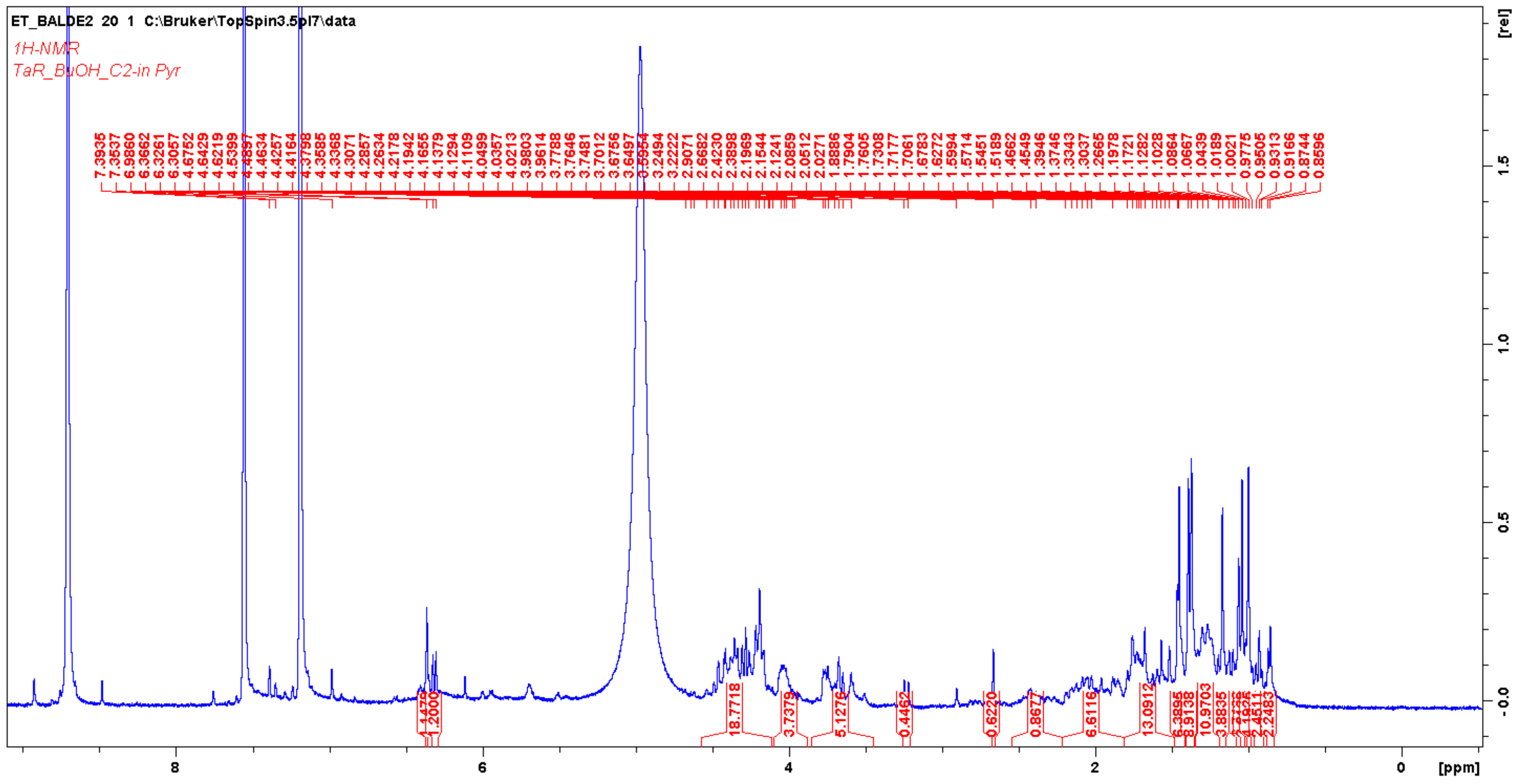

Figure S7. ${ }^{1} \mathrm{H}$ NMR spectrum (pyridine- $d_{5}, 400 \mathrm{MHz}$ ) of compound (2) 


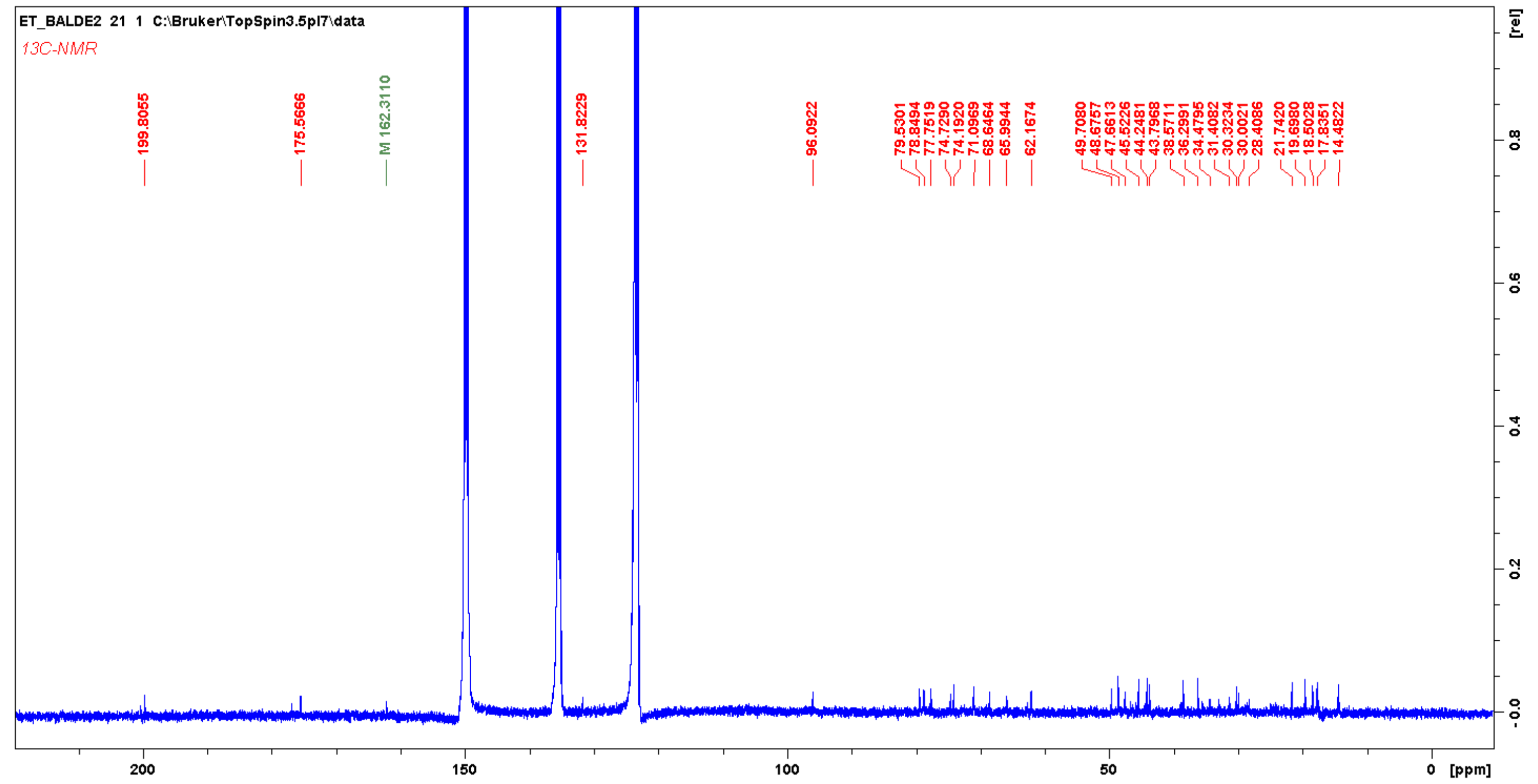

Figure S8. ${ }^{13} \mathrm{C}$ NMR spectrum (pyridine- $d_{5}, 100 \mathrm{MHz}$ ) of compound (2) 


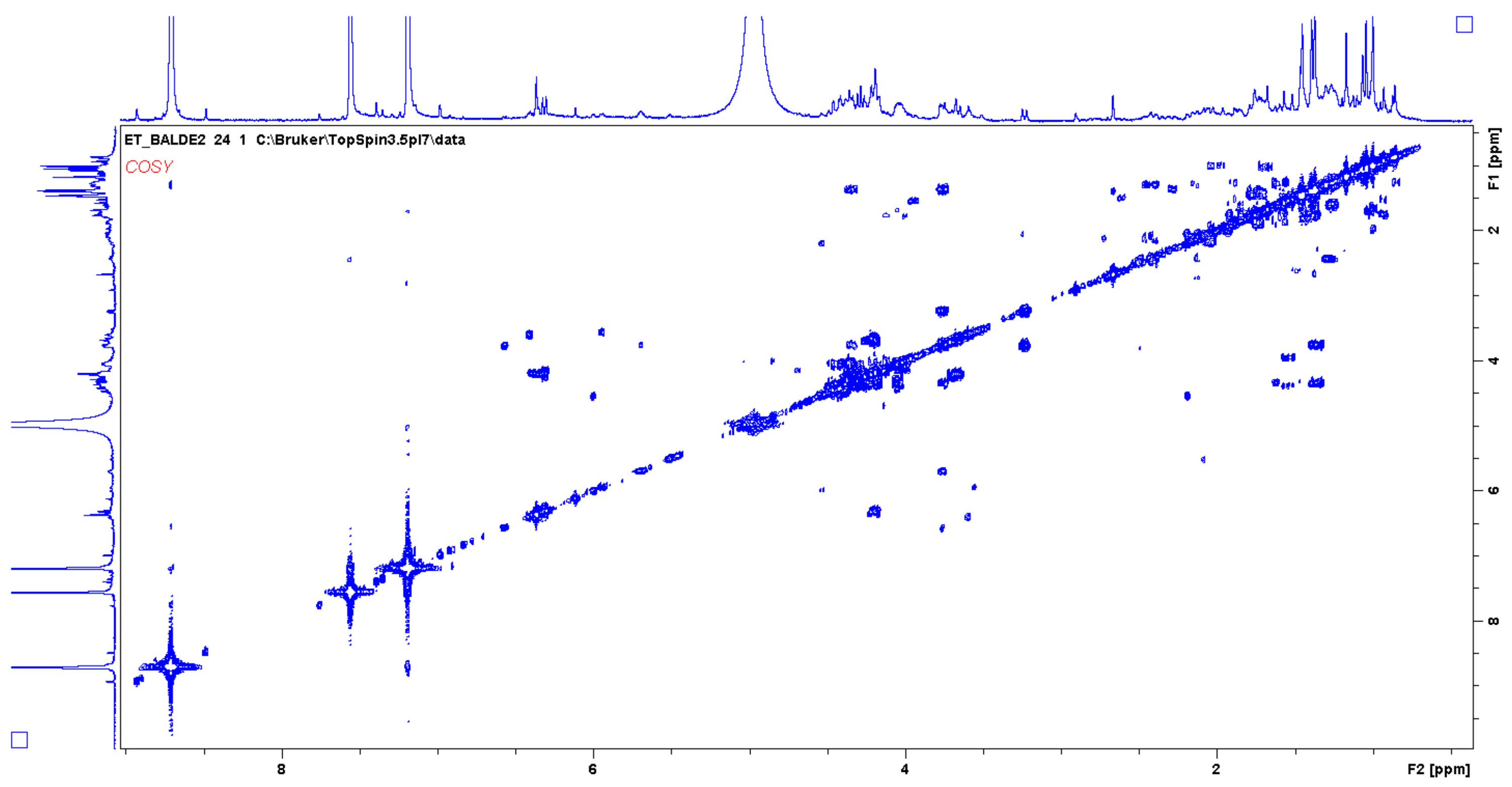

Figure S9. COSY spectrum (pyridine- $d_{5}$ ) of compound (2) 


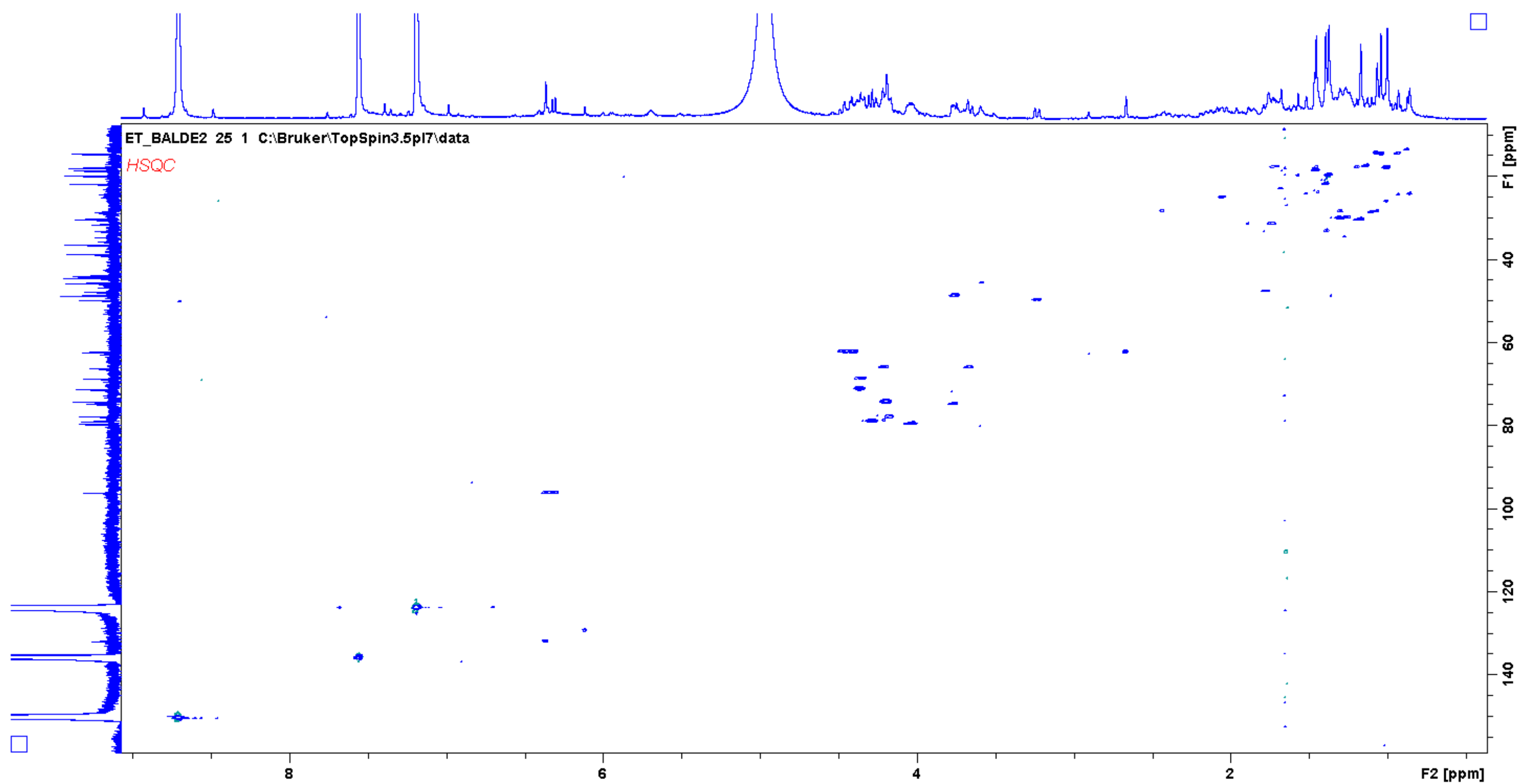

Figure S10. HSQC spectrum (pyridine- $d_{5}$ ) of compound (2) 


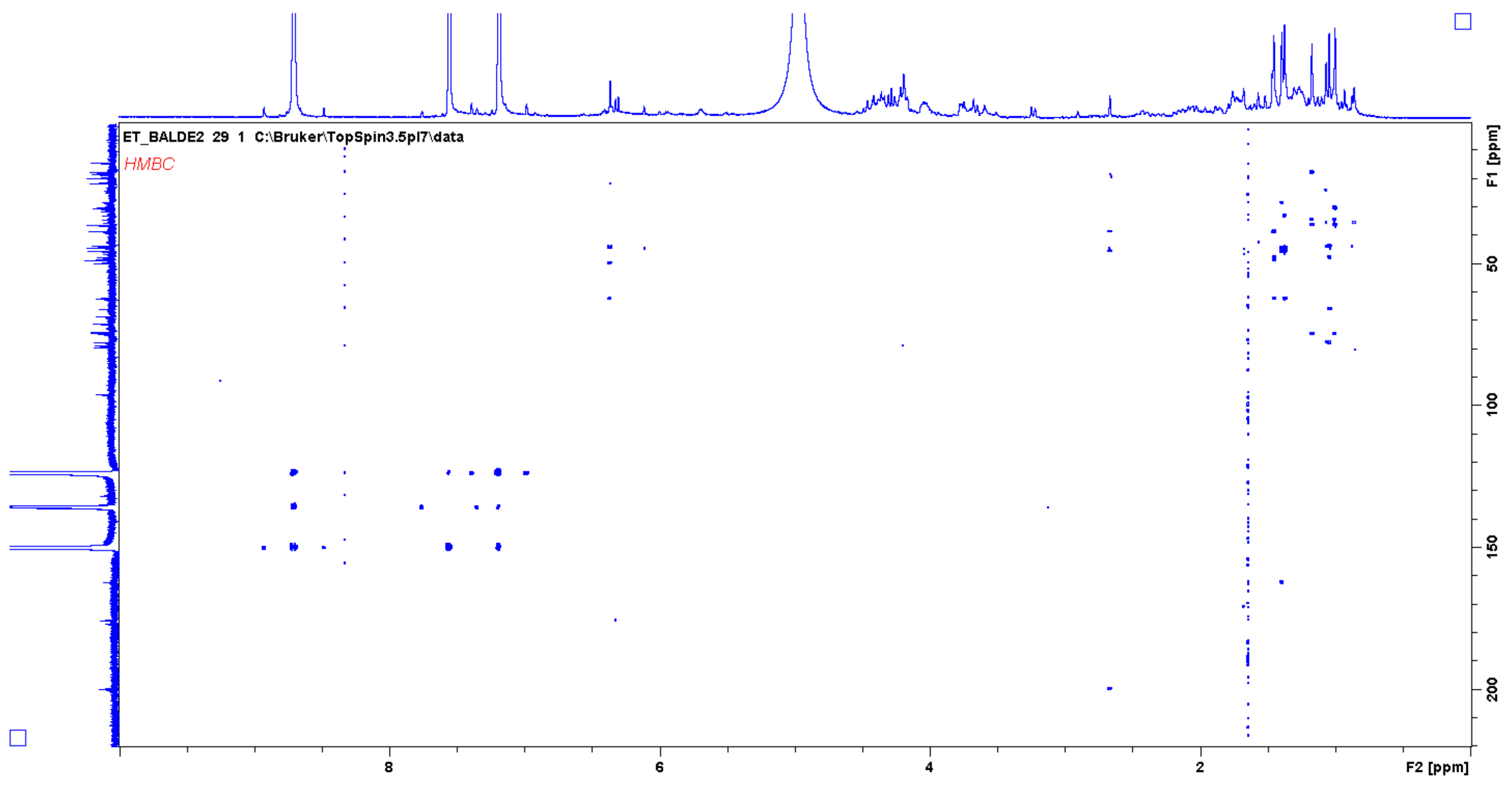

Figure S11. HMBC spectrum (pyridine- $d_{5}$ ) of compound (2) 


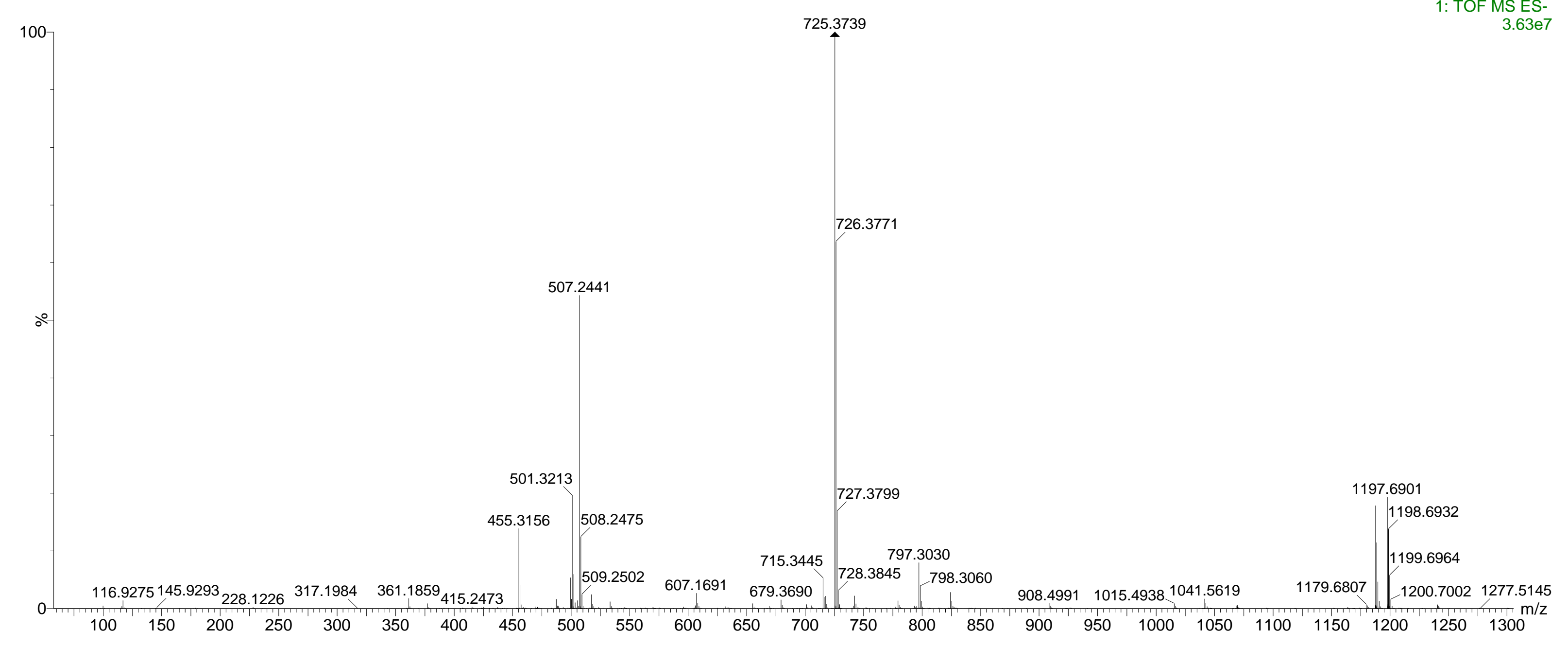

Figure S12. HR-ESI-MS spectrum of compound (2) 


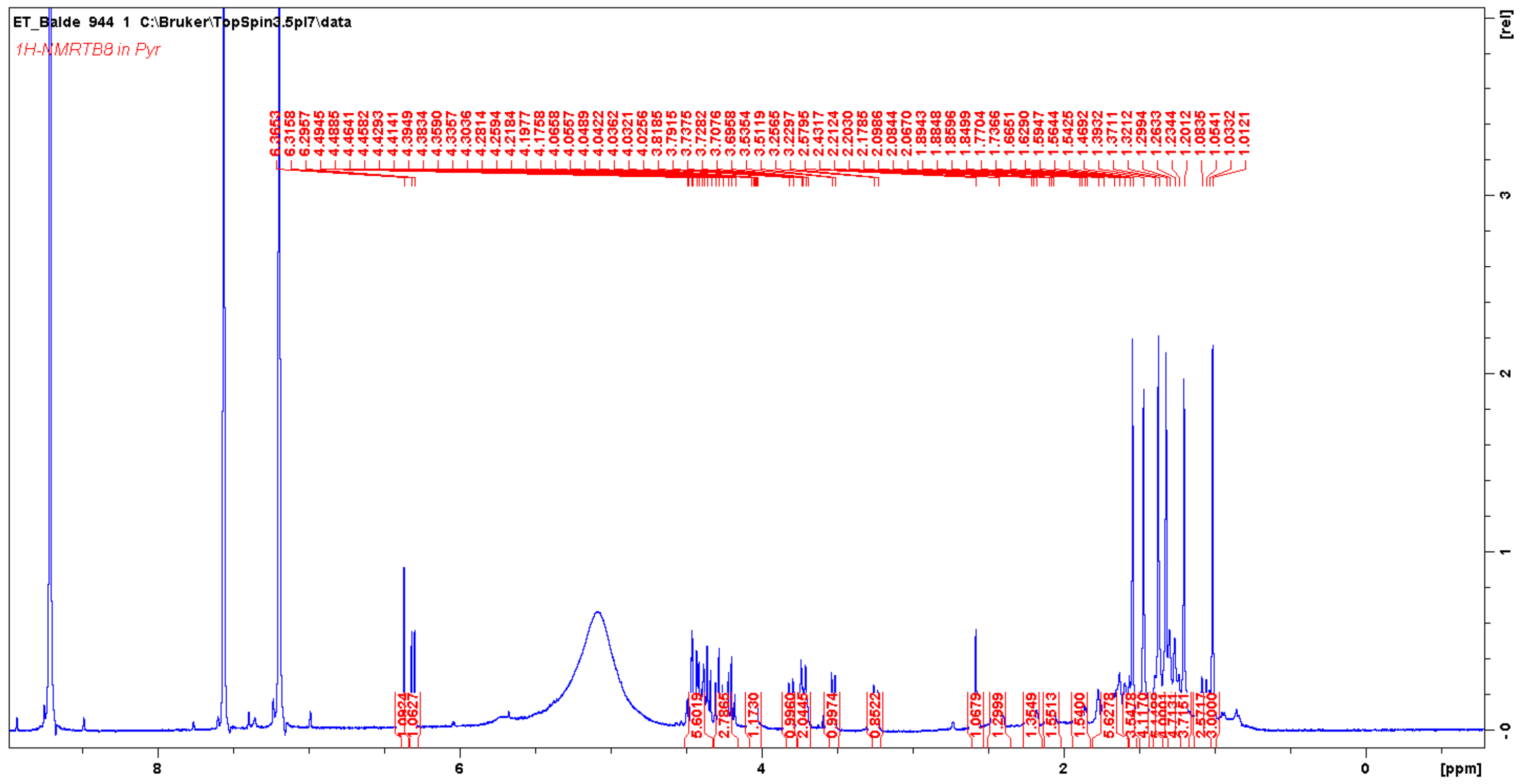

Figure S13. ${ }^{1} \mathrm{H}$ NMR spectrum (pyridine- $d_{5}, 400 \mathrm{MHz}$ ) of compound (3) 


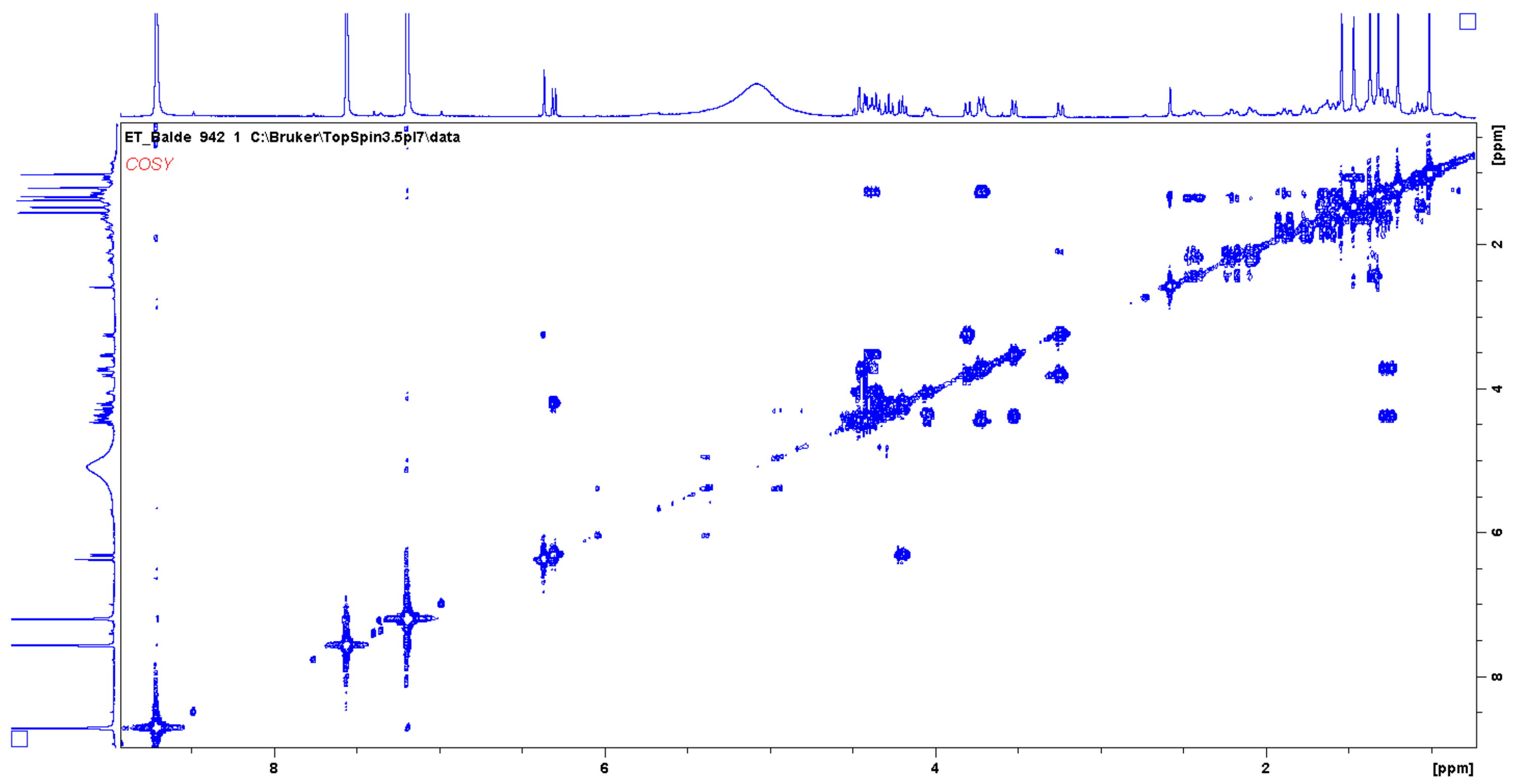

Figure S14. COSY spectrum (pyridine- $d_{5}$ ) of compound (3) 


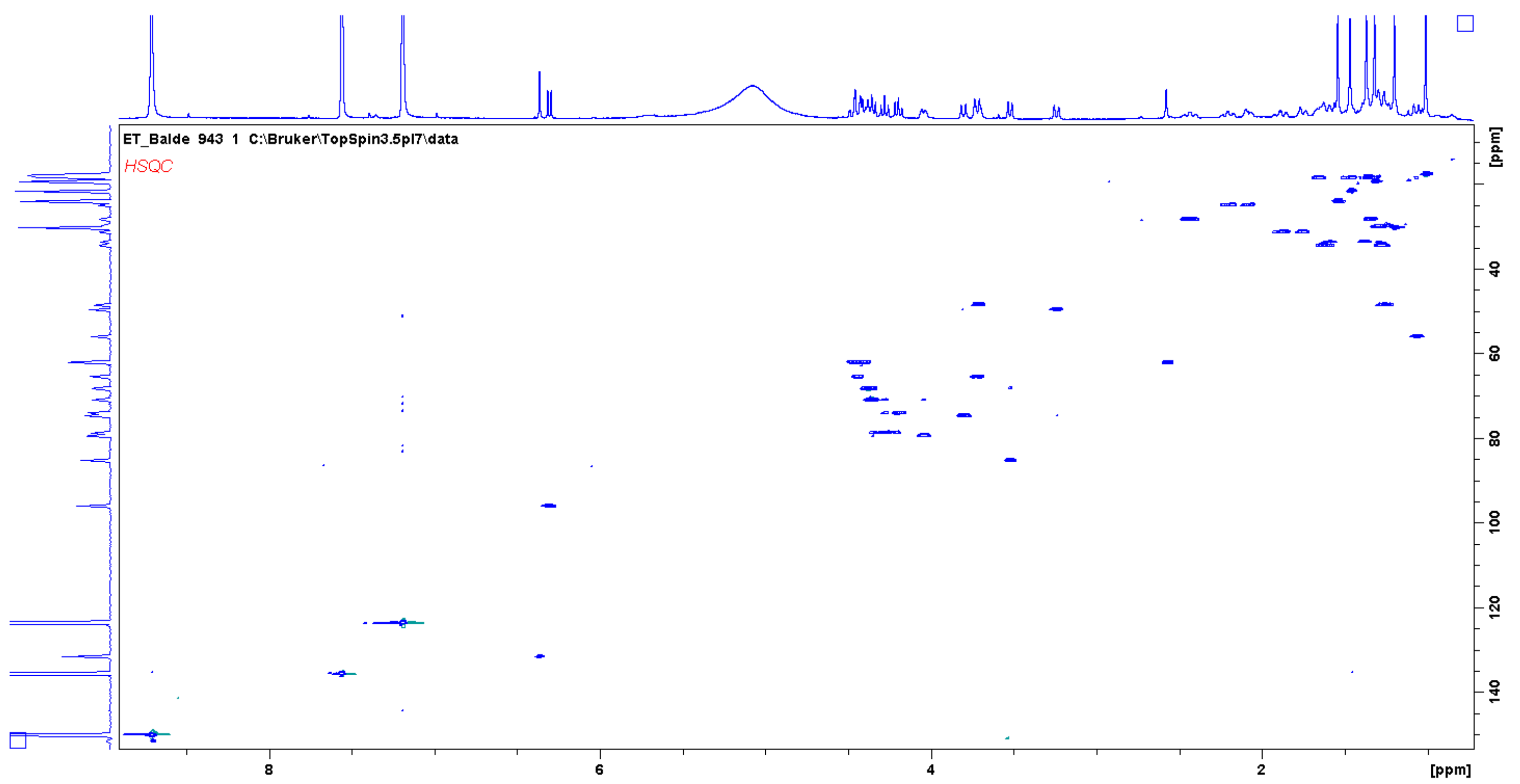

Figure S15. HSQC spectrum (pyridine- $d_{5}$ ) of compound (3) 


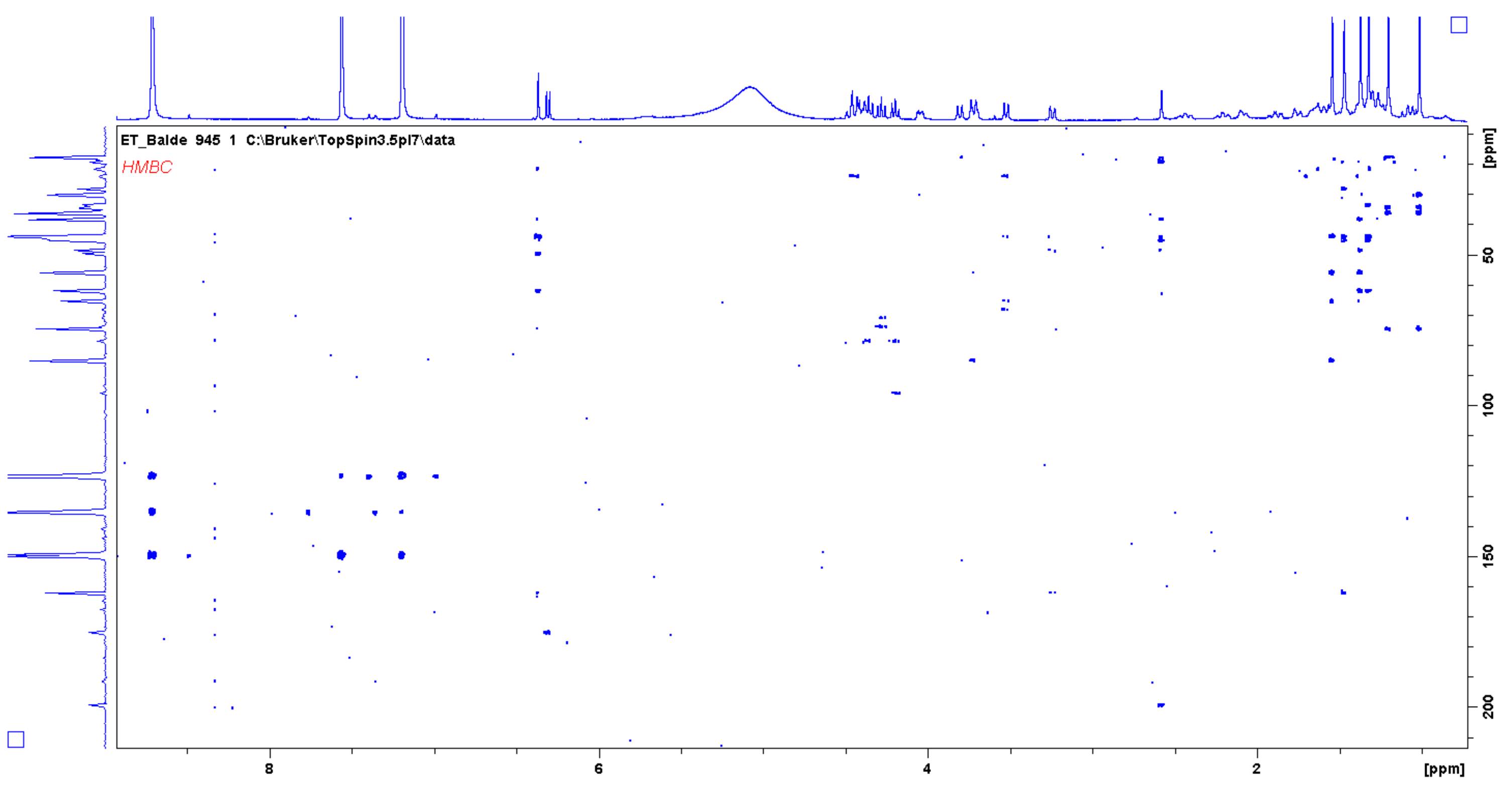

Figure S16. HMBC spectrum (pyridine- $d_{5}$ ) of compound (3) 


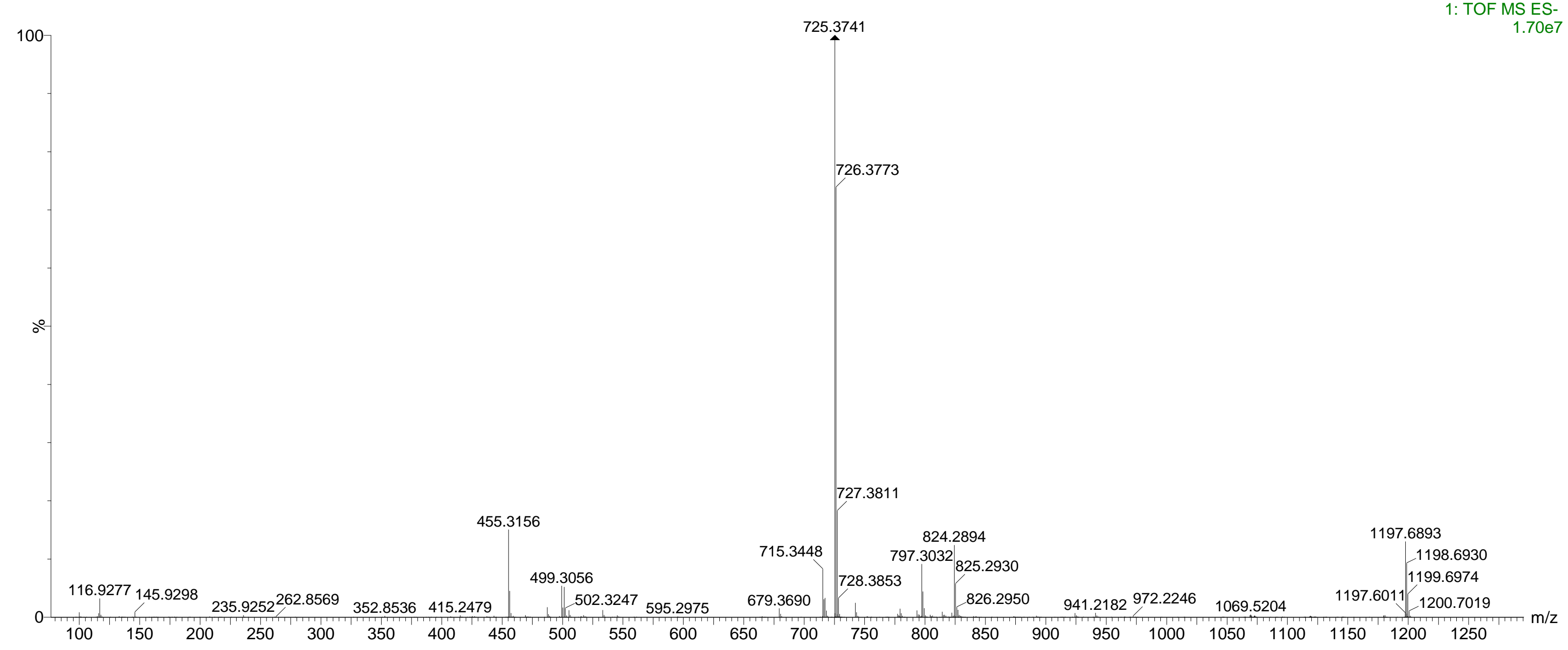

Figure S17. HR-ESI-MS spectrum of compound (3) 


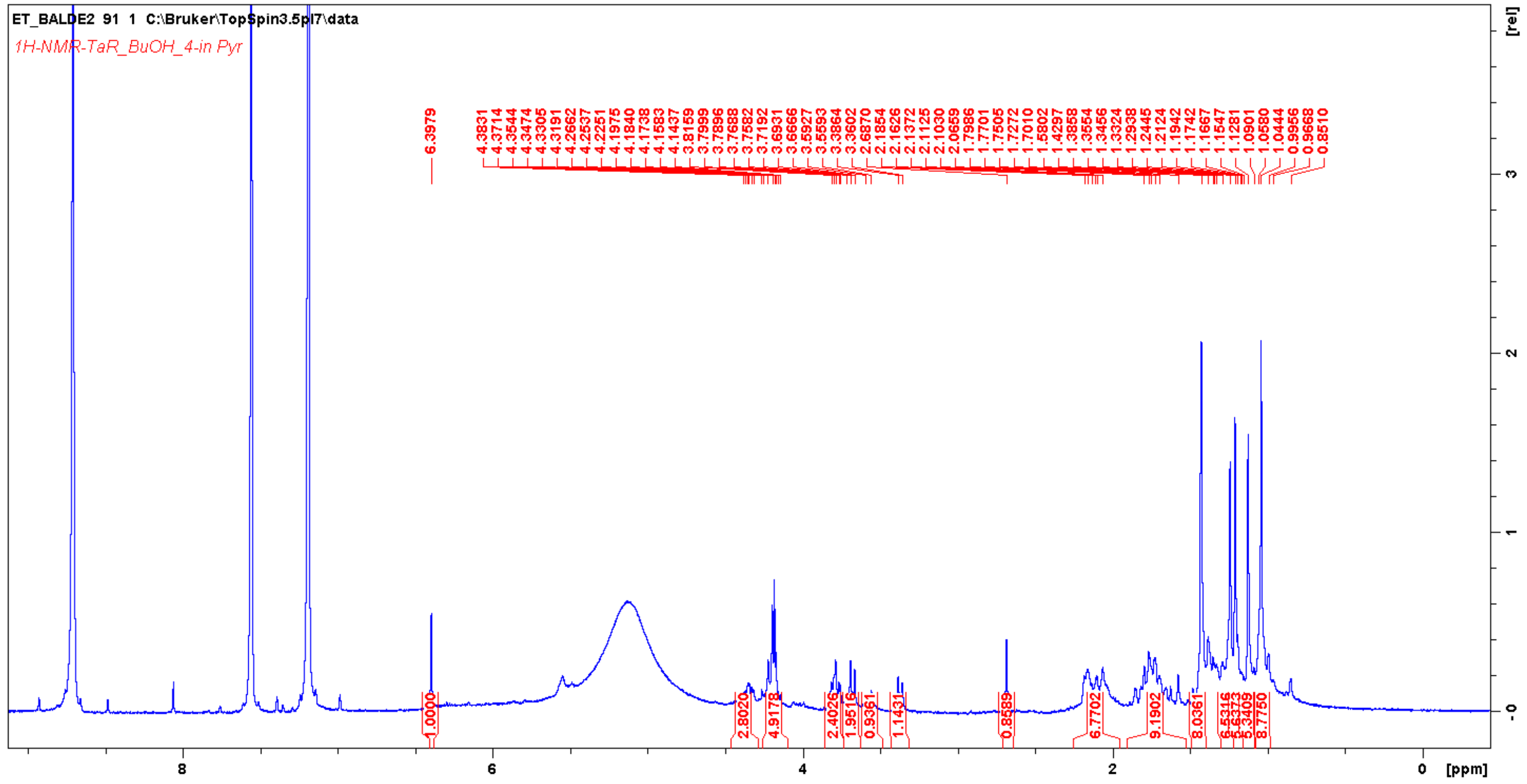

Figure S18. ${ }^{1} \mathrm{H}$ NMR spectrum (pyridine- $d_{5}, 400 \mathrm{MHz}$ ) of compound (4) 


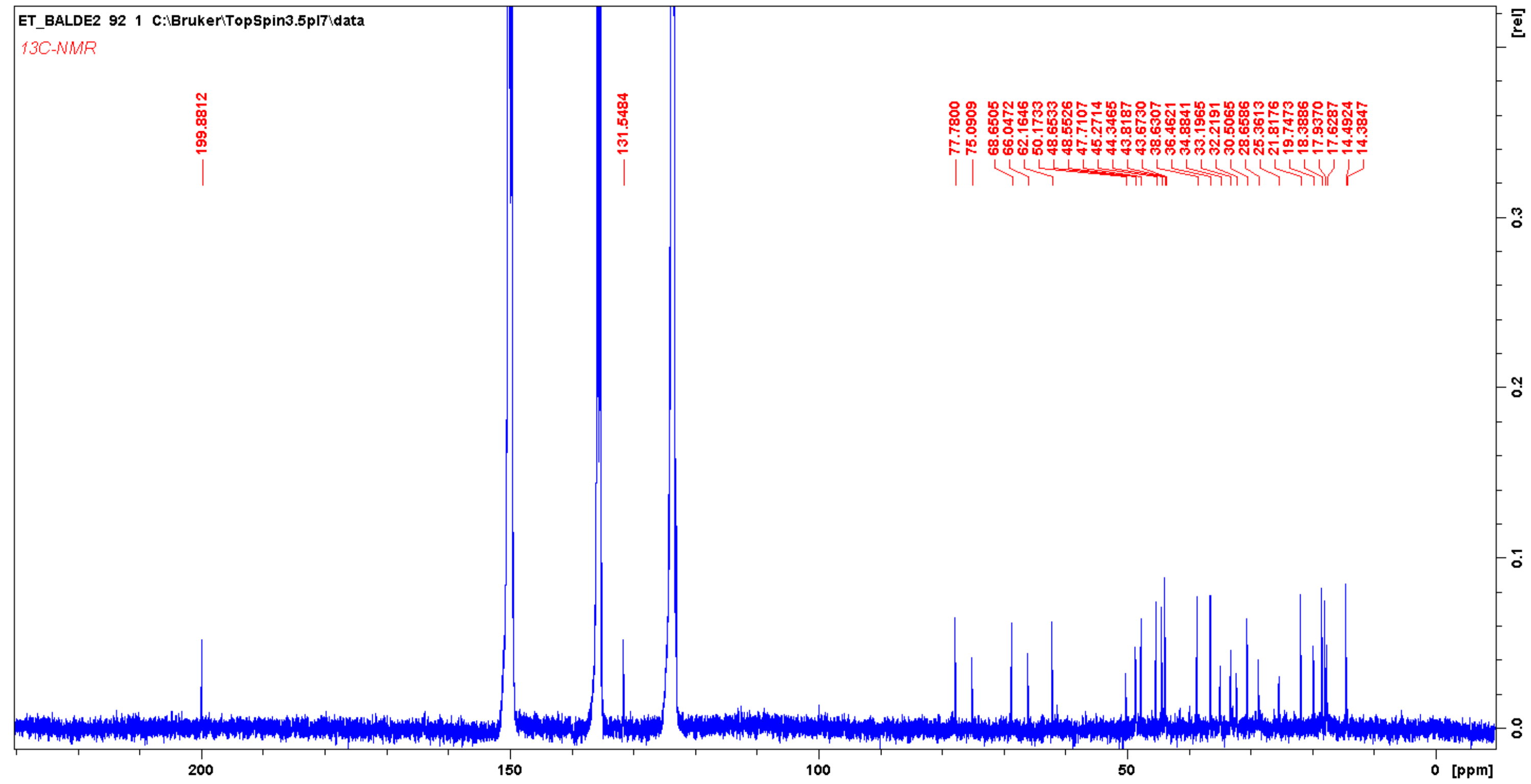

Figure S19. ${ }^{13} \mathrm{C}$ NMR spectrum (pyridine- $d_{5}, 100 \mathrm{MHz}$ ) of compound (4) 


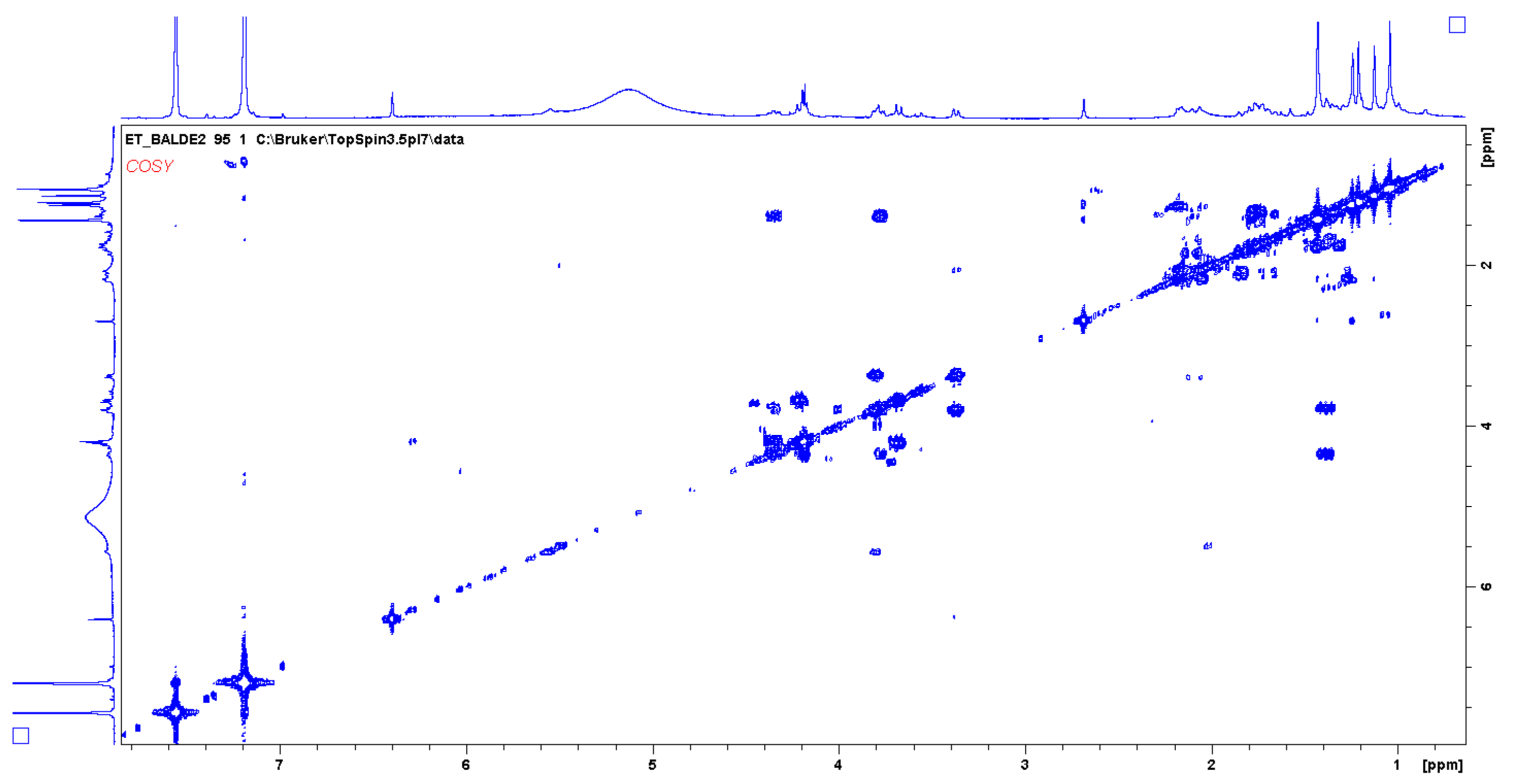

Figure S20. COSY spectrum (pyridine- $d_{5}$ ) of compound (4) 


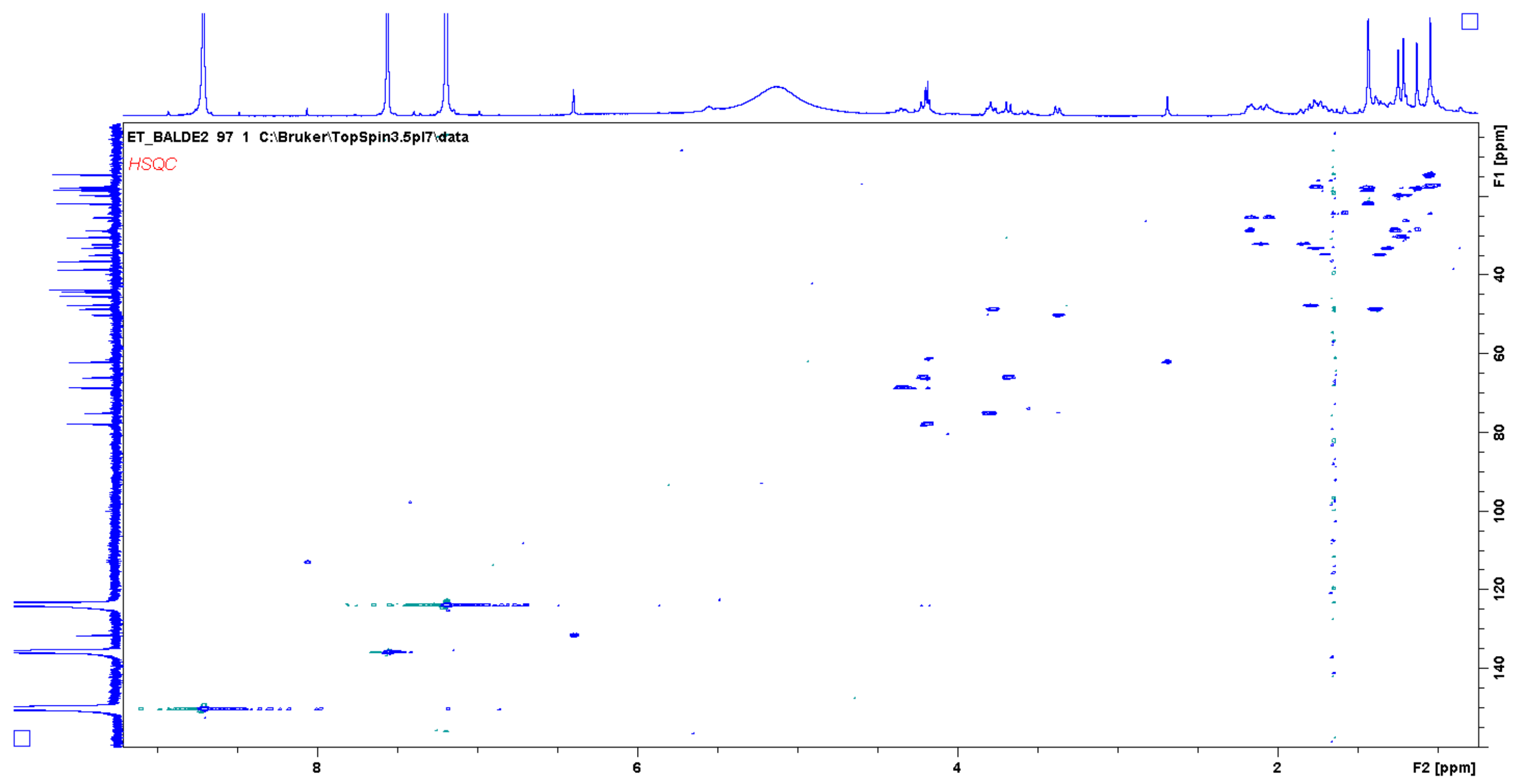

Figure S21. HSQC spectrum (pyridine- $d_{5}$ ) of compound (4) 


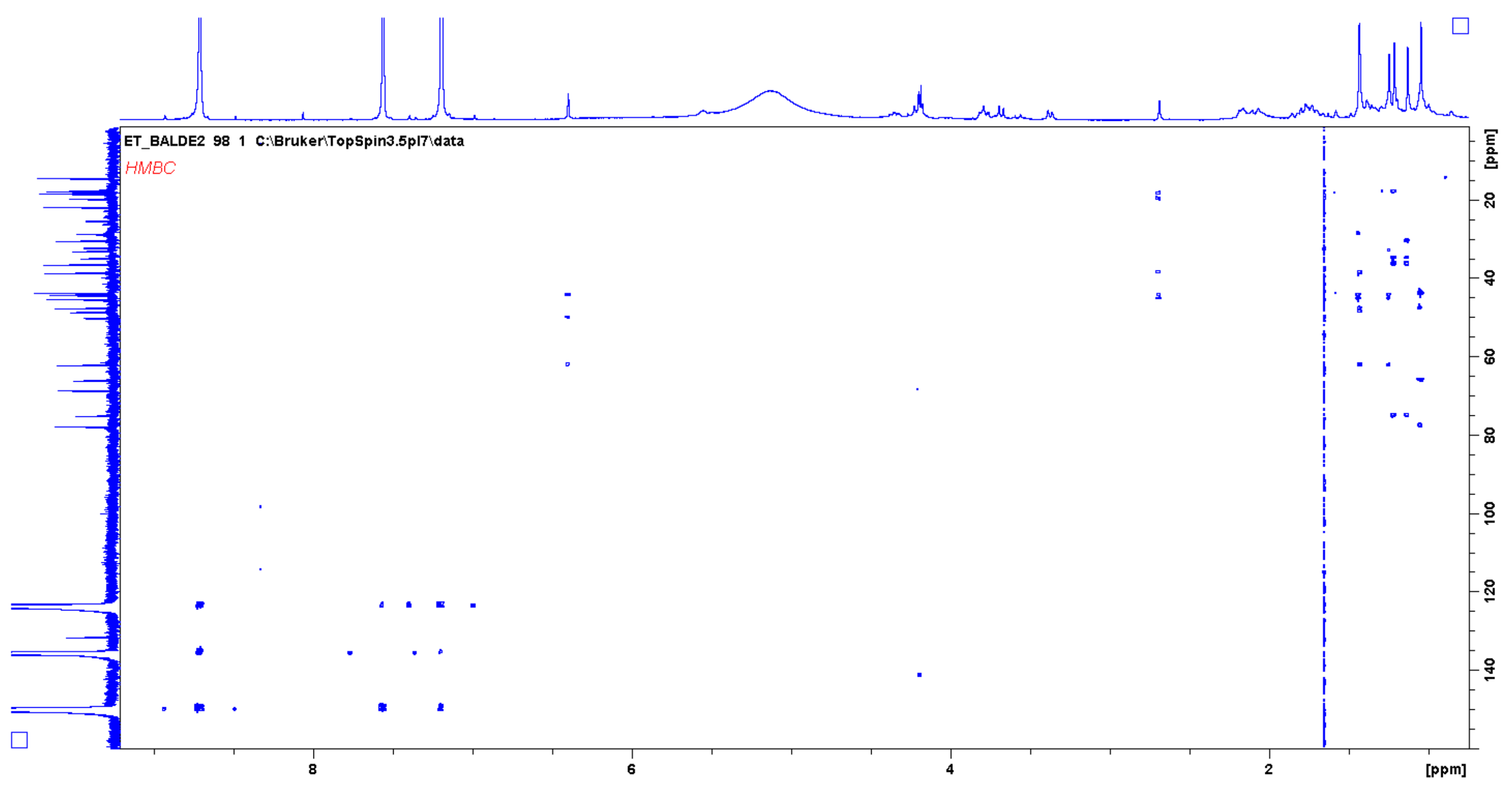

Figure S22. HMBC spectrum (pyridine- $d_{5}$ ) of compound (4) 


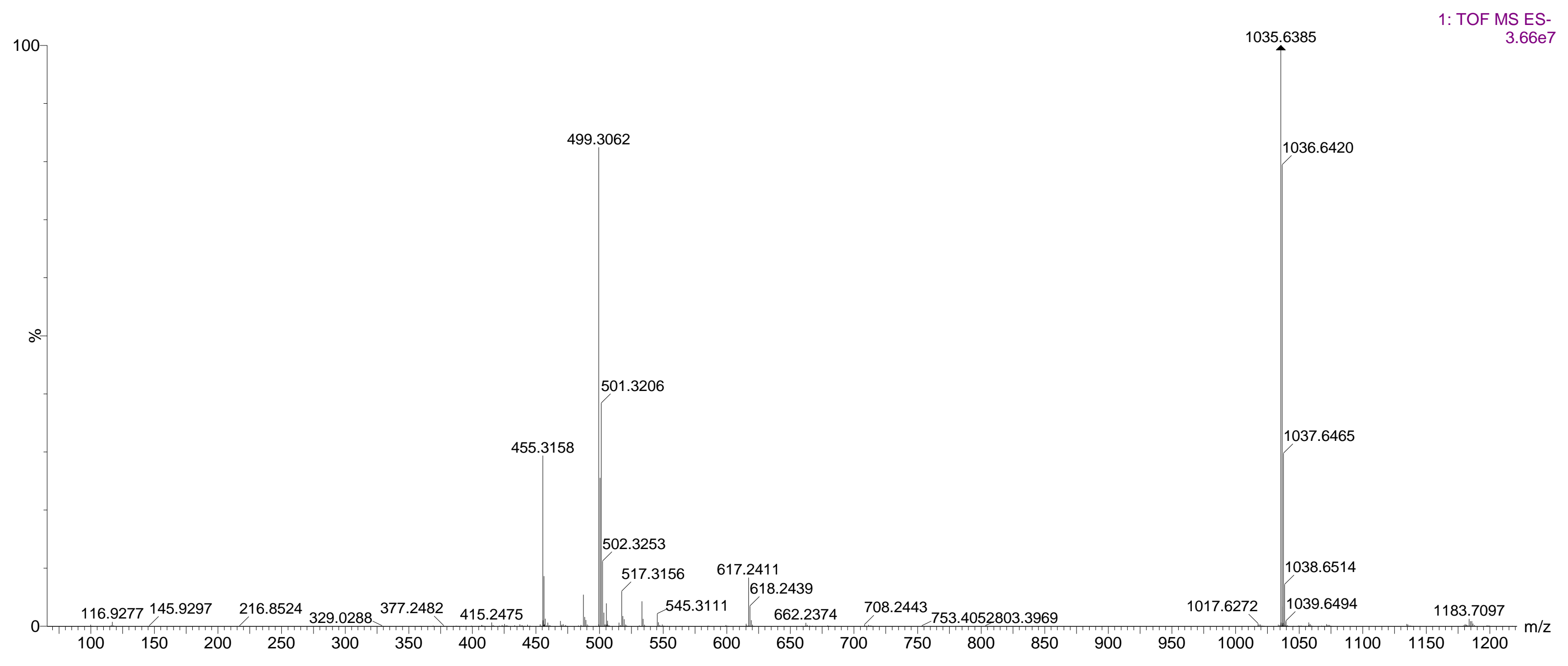

Figure S23. HR-ESI-MS spectrum of compound (4) 


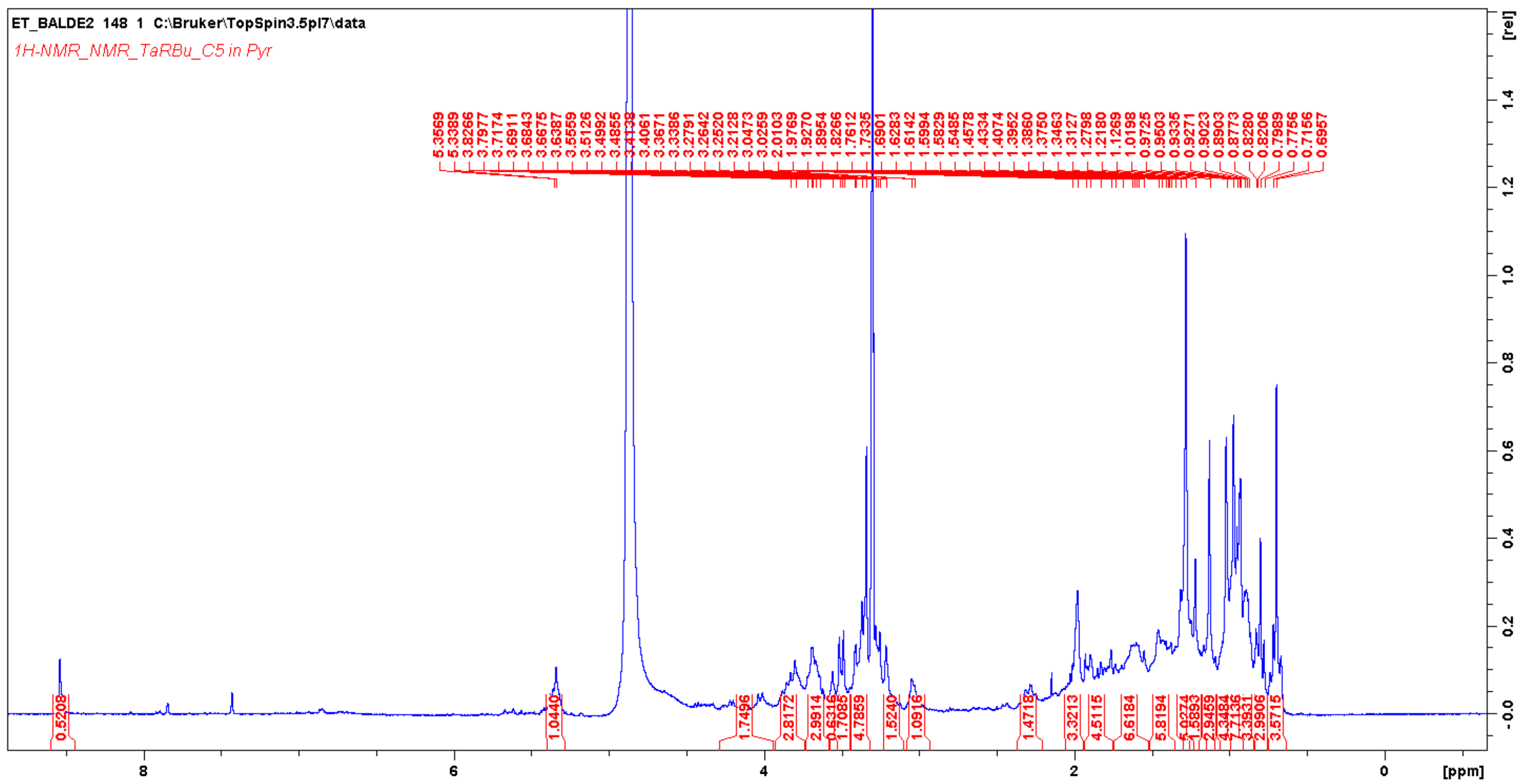

Figure S24. ${ }^{1} \mathrm{H}$ NMR spectrum (methanol- $d_{4}, 400 \mathrm{MHz}$ ) of compound (5) 


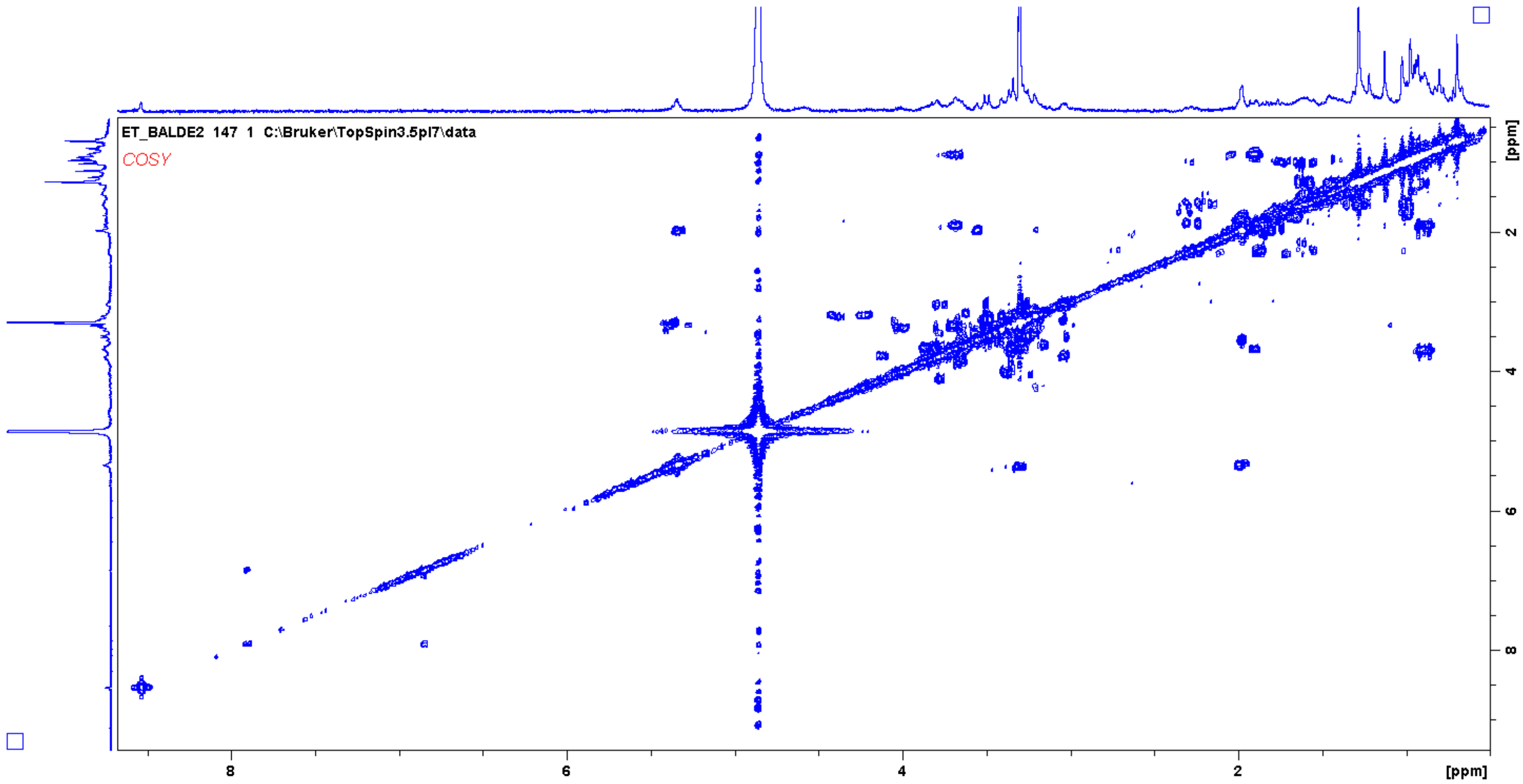

Figure S25. COSY spectrum (methanol- $d_{4}$ ) of compound (5) 


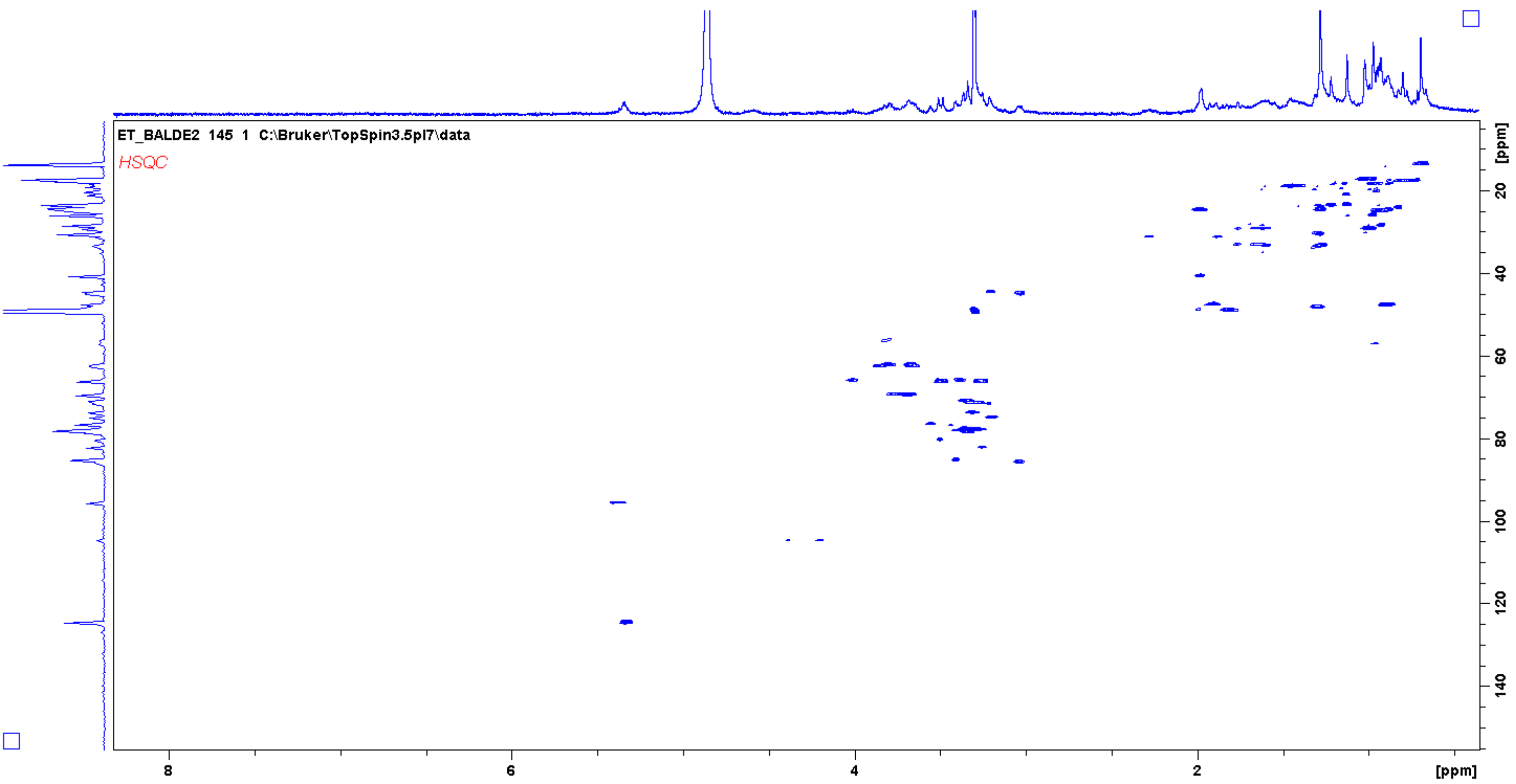

Figure S26. HSQC spectrum (methanol- $d_{4}$ ) of compound (5) 


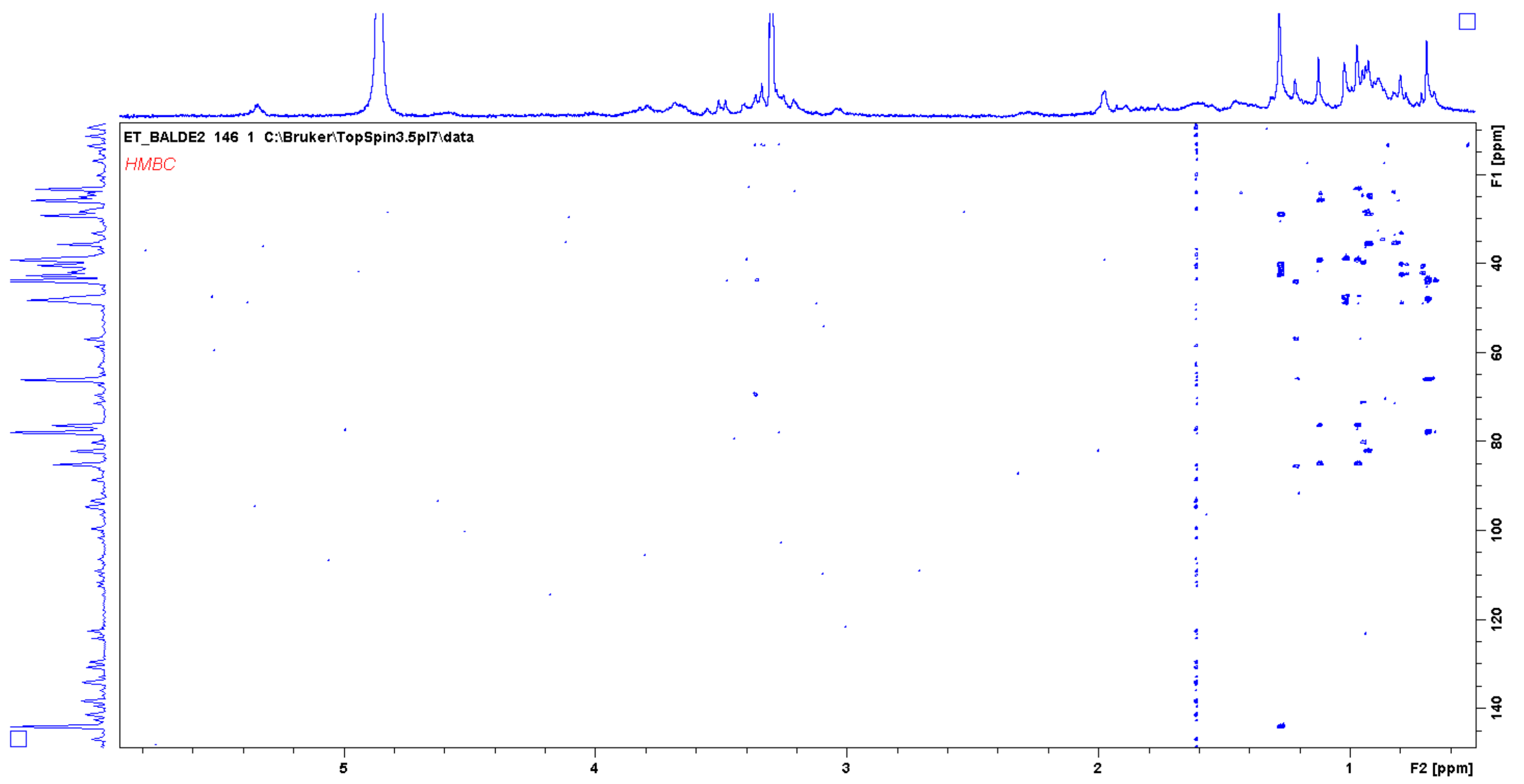

Figure S27. HMBC spectrum (methanol- $d_{4}$ ) of compound (5) 


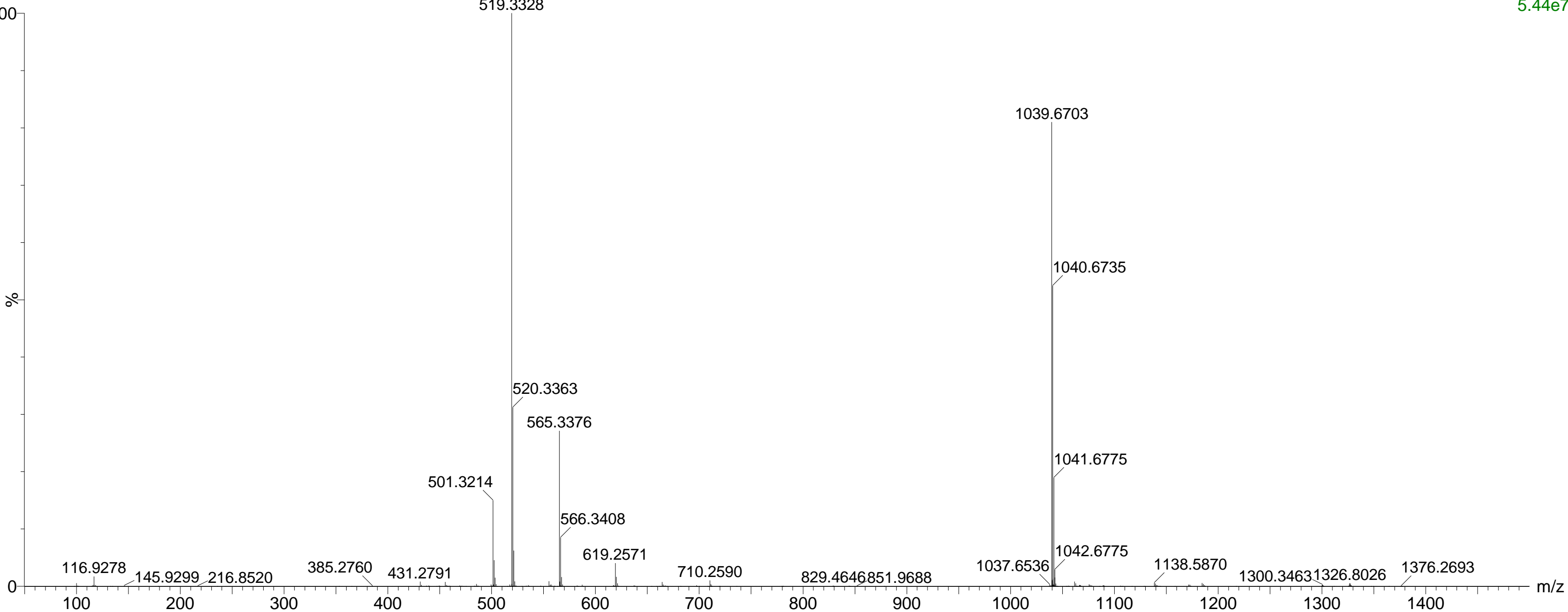

\section{Figure S28. HR-ESI-MS spectrum of compound (5)}




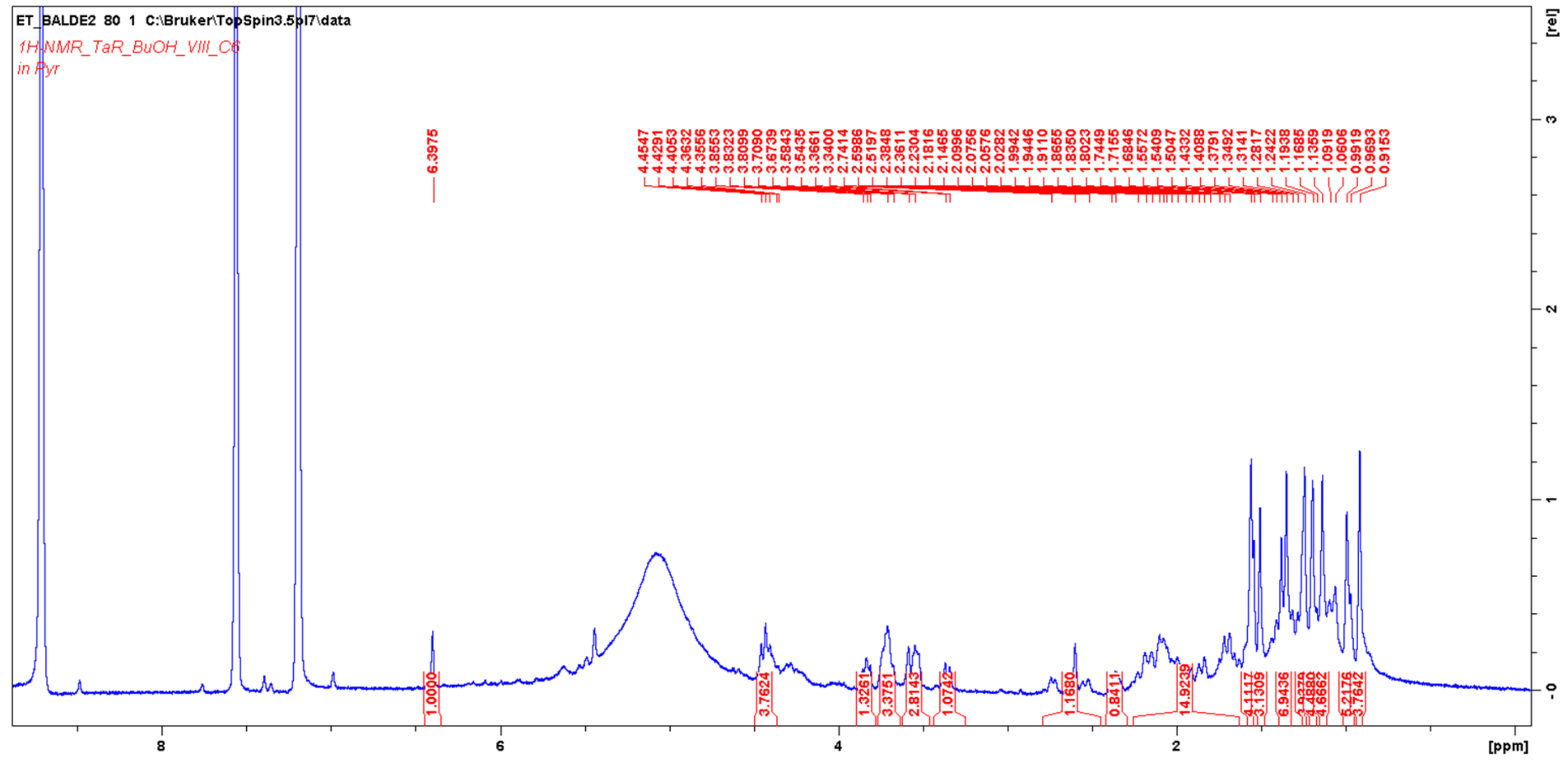

Figure S29. ${ }^{1} \mathrm{H}$ NMR spectrum (pyridine- $d_{5}, 400 \mathrm{MHz}$ ) of compound (6) 


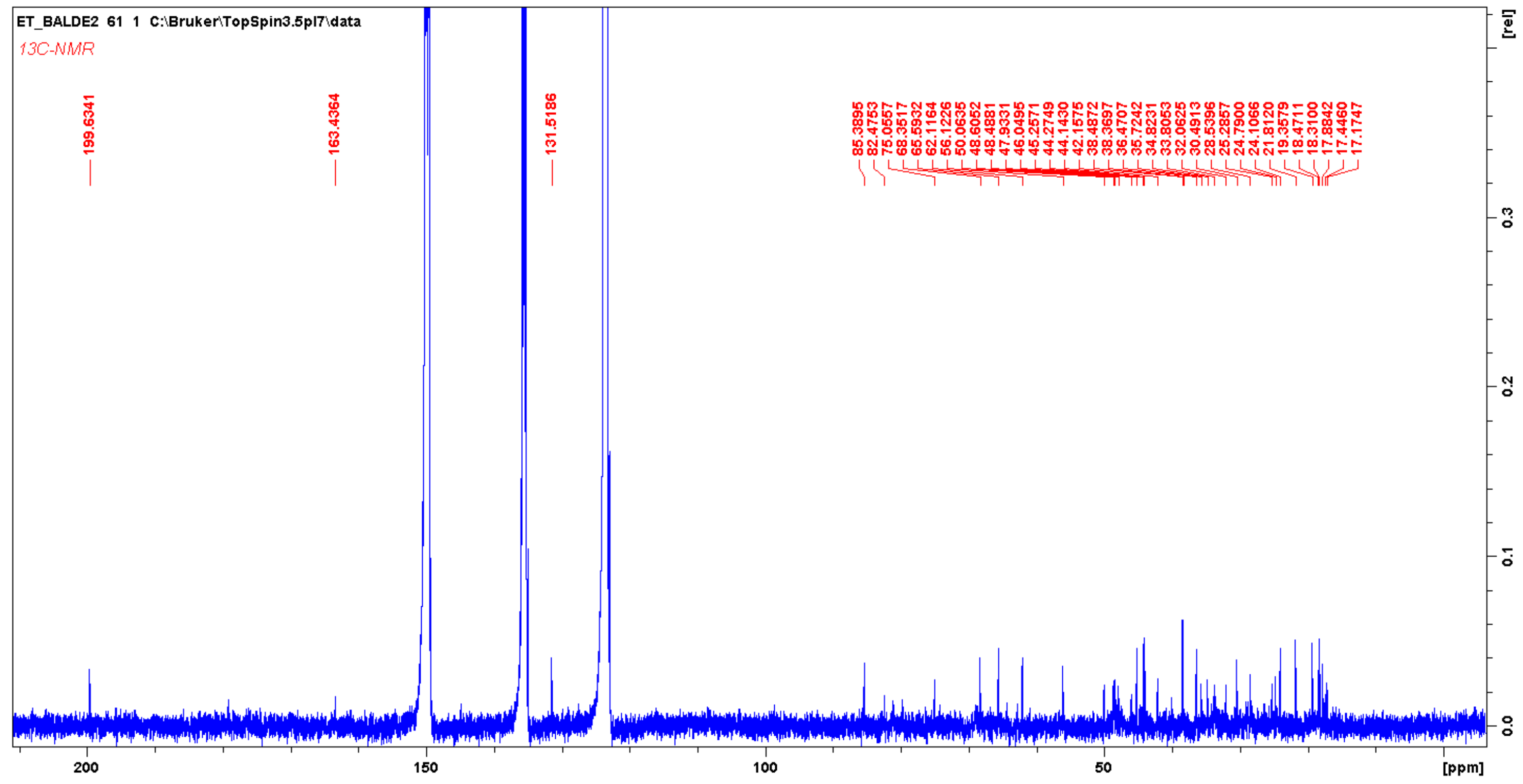

Figure S30. ${ }^{13} \mathrm{C}$ NMR spectrum (pyridine- $d 5,100 \mathrm{MHz}$ ) of compound (6) 


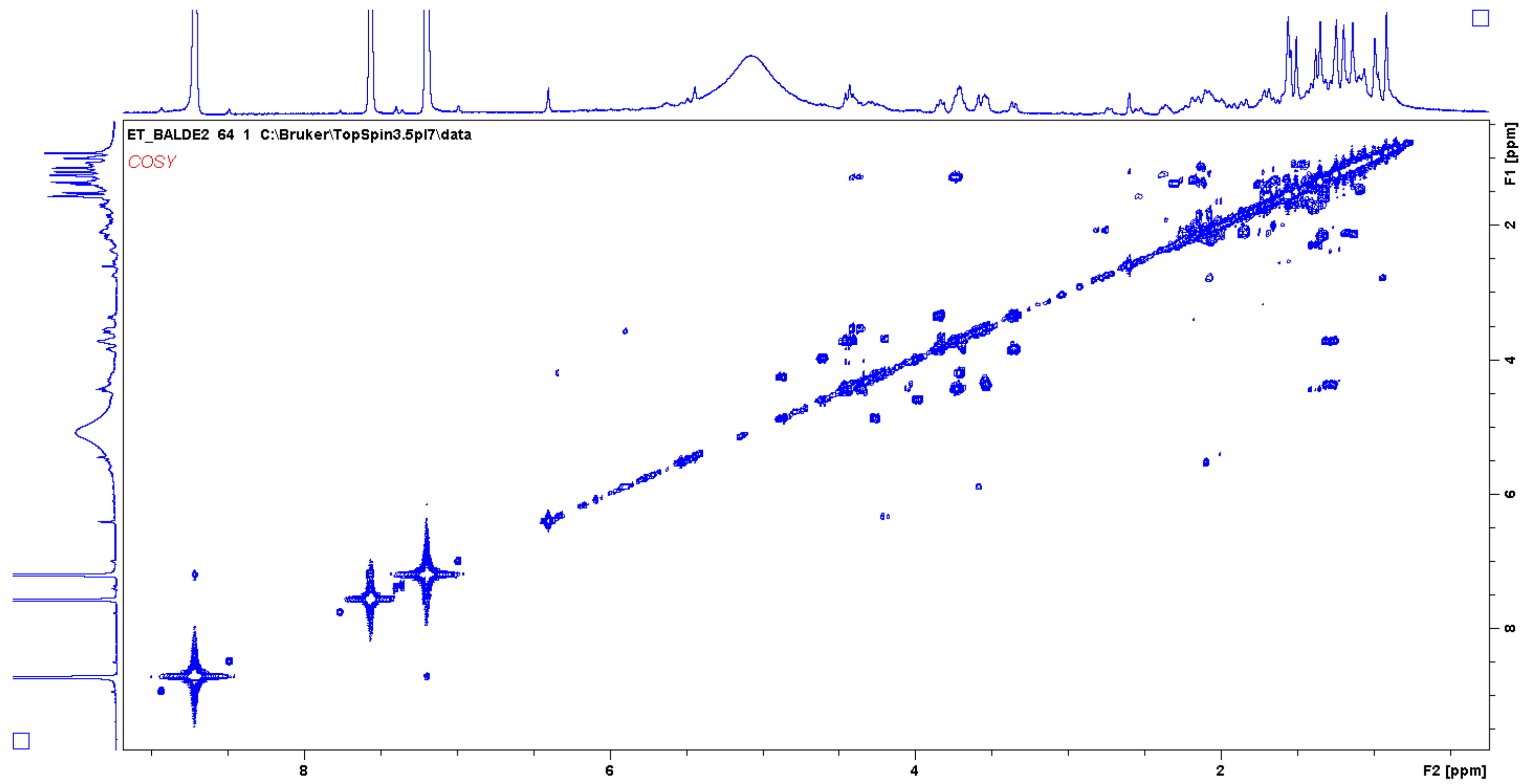

Figure S31. COSY spectrum (pyridine- $d_{5}$ ) of compound (6) 


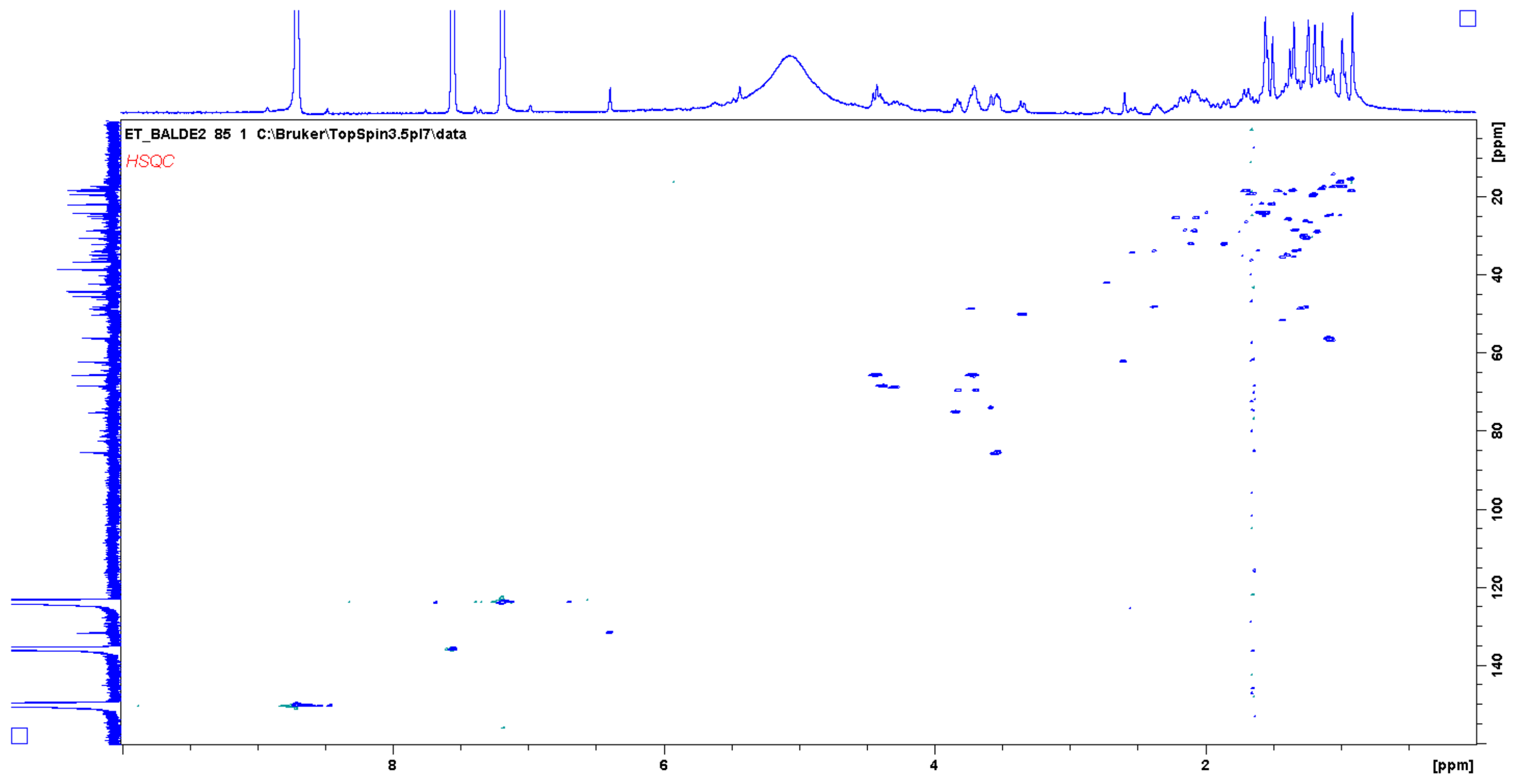

Figure S32. HSQC spectrum (pyridine- $d_{5}$ ) of compound (6) 


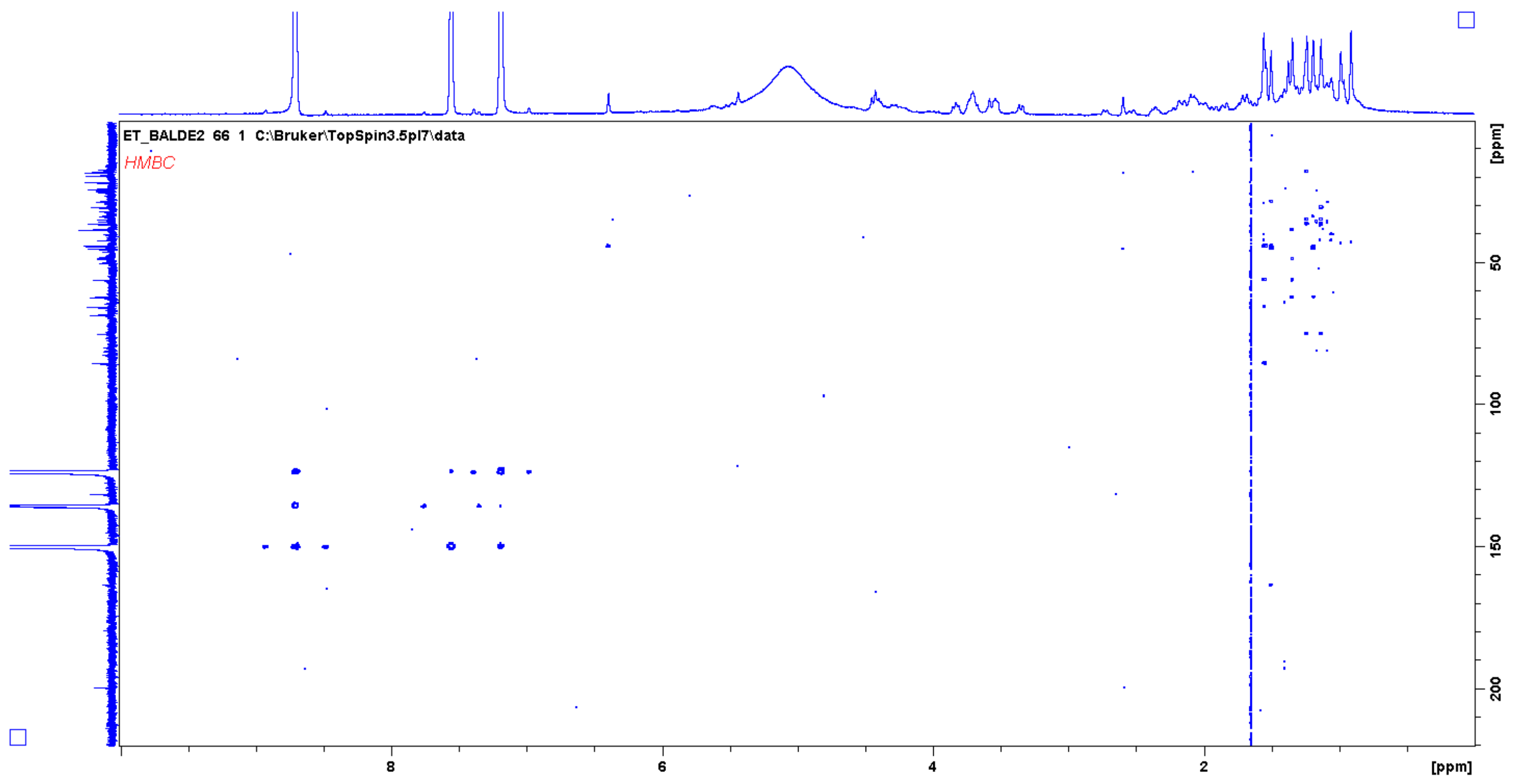

Figure S33. HMBC spectrum (pyridine- $d_{5}$ ) of compound (6) 


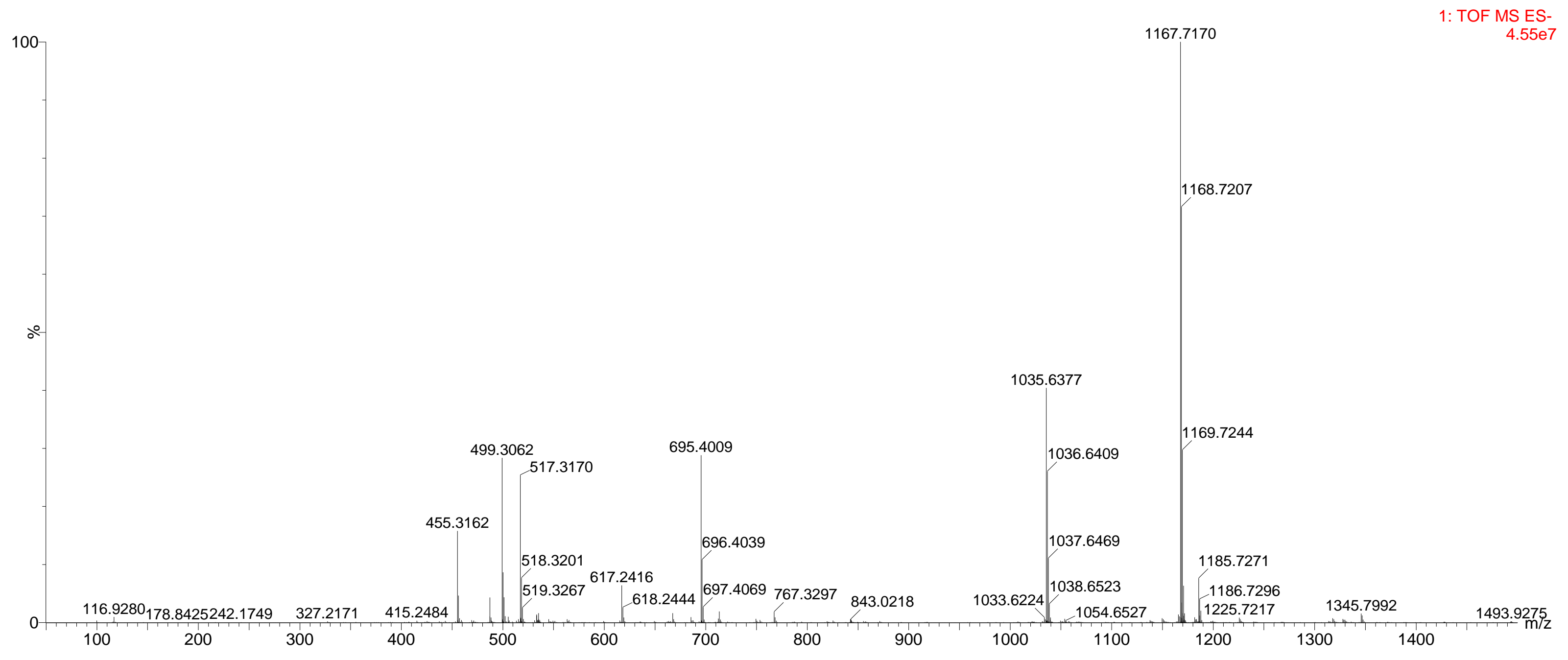

Figure S34. HR-ESI-MS spectrum of compound (6) 


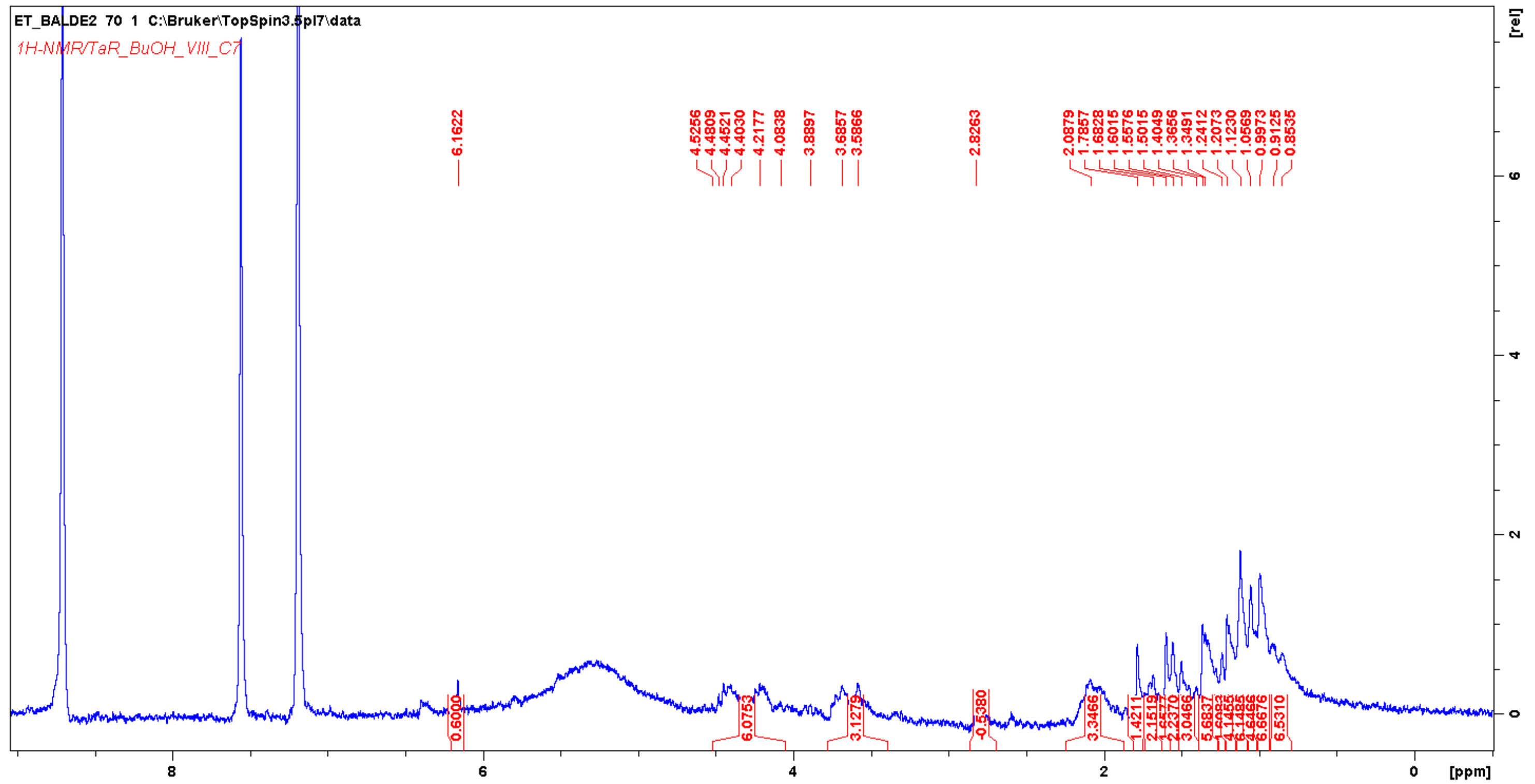

Figure S35. ${ }^{1} \mathrm{H}$ NMR spectrum (pyridine- $d_{5}$ ) of compound (7) 


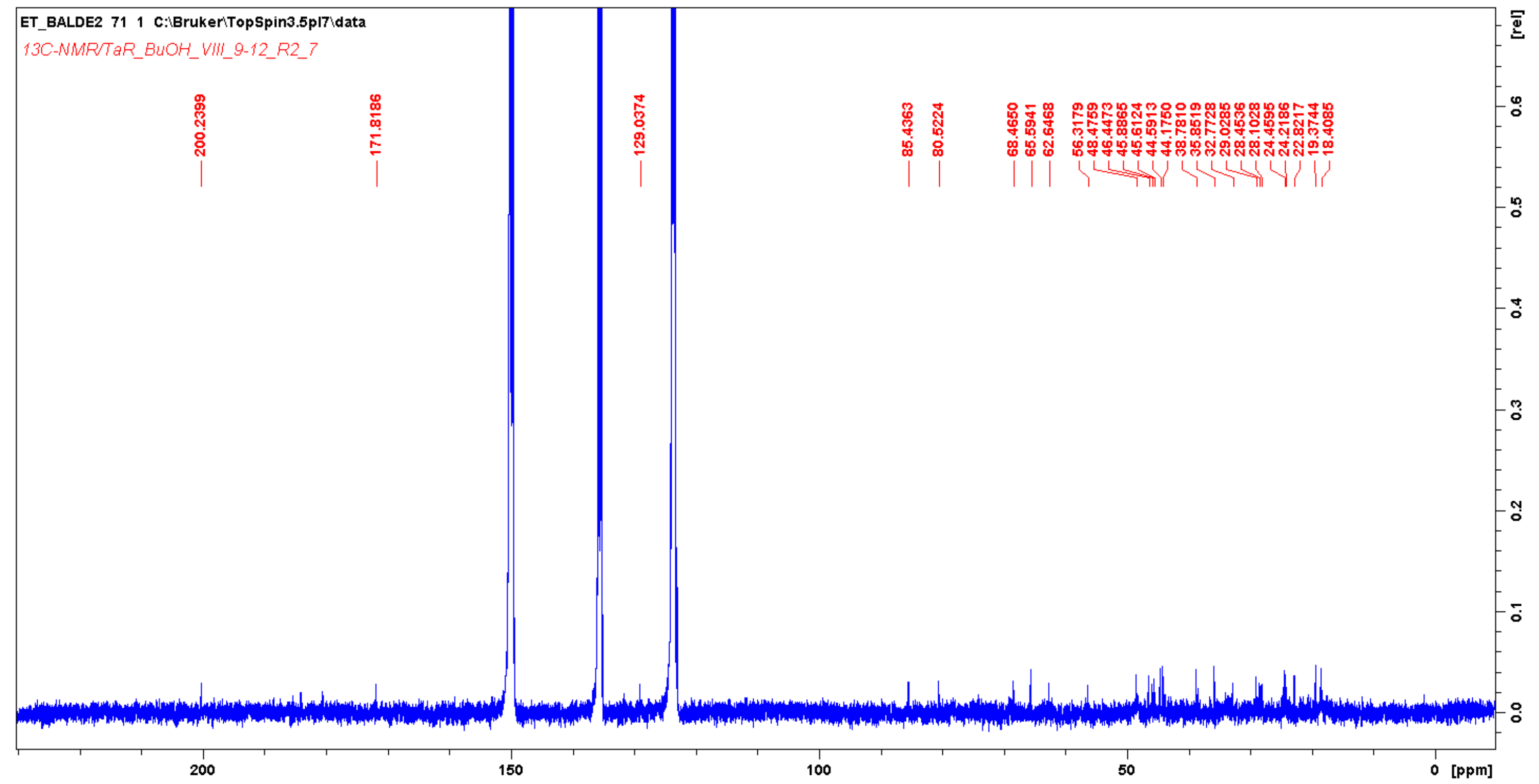

Figure S36. ${ }^{13} \mathrm{C}$ NMR spectrum (pyridine- $d_{5}, 100 \mathrm{MHz}$ ) of compound (7) 


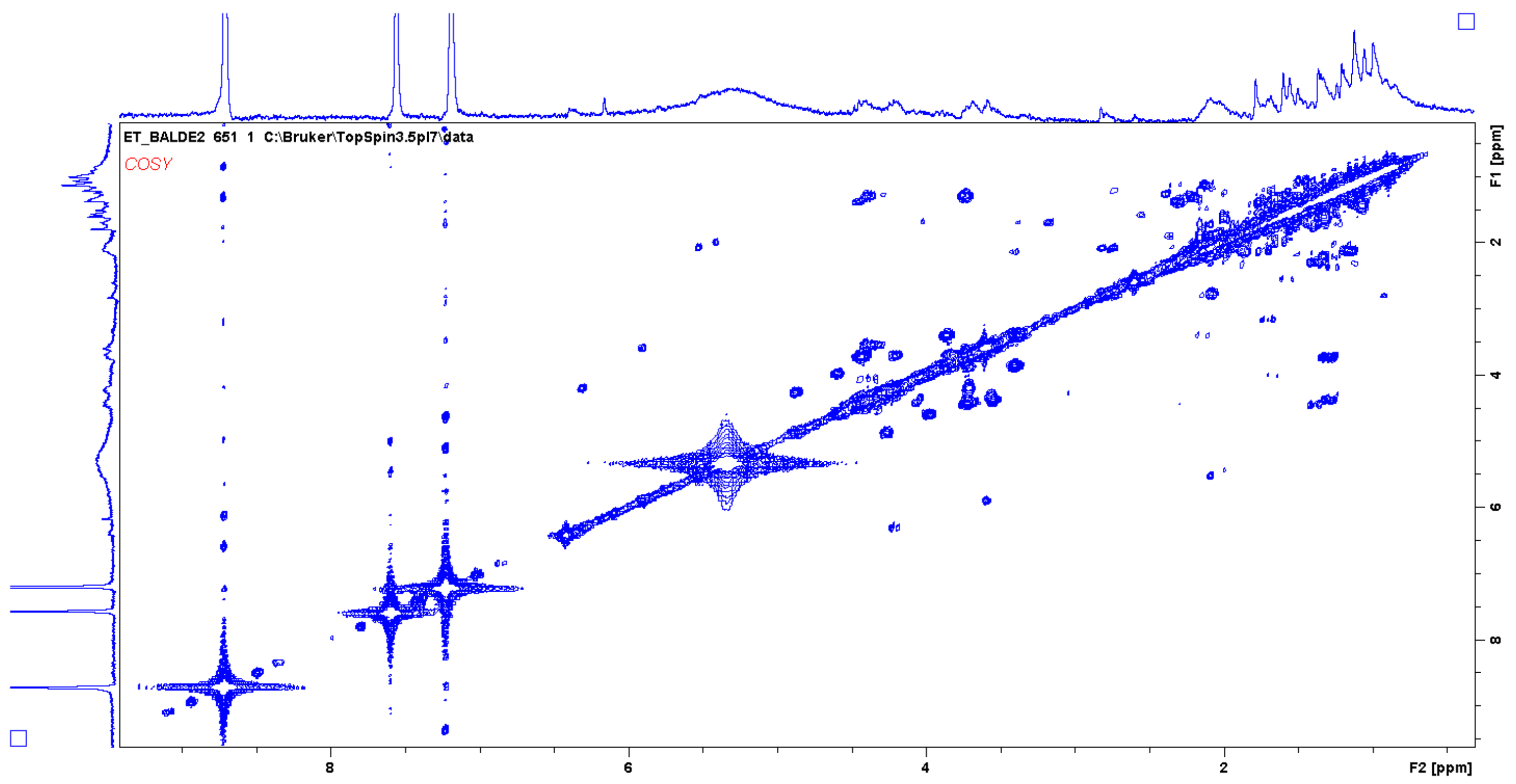

Figure S37. COSY spectrum (pyridine- $d_{5}$ ) of compound (7) 


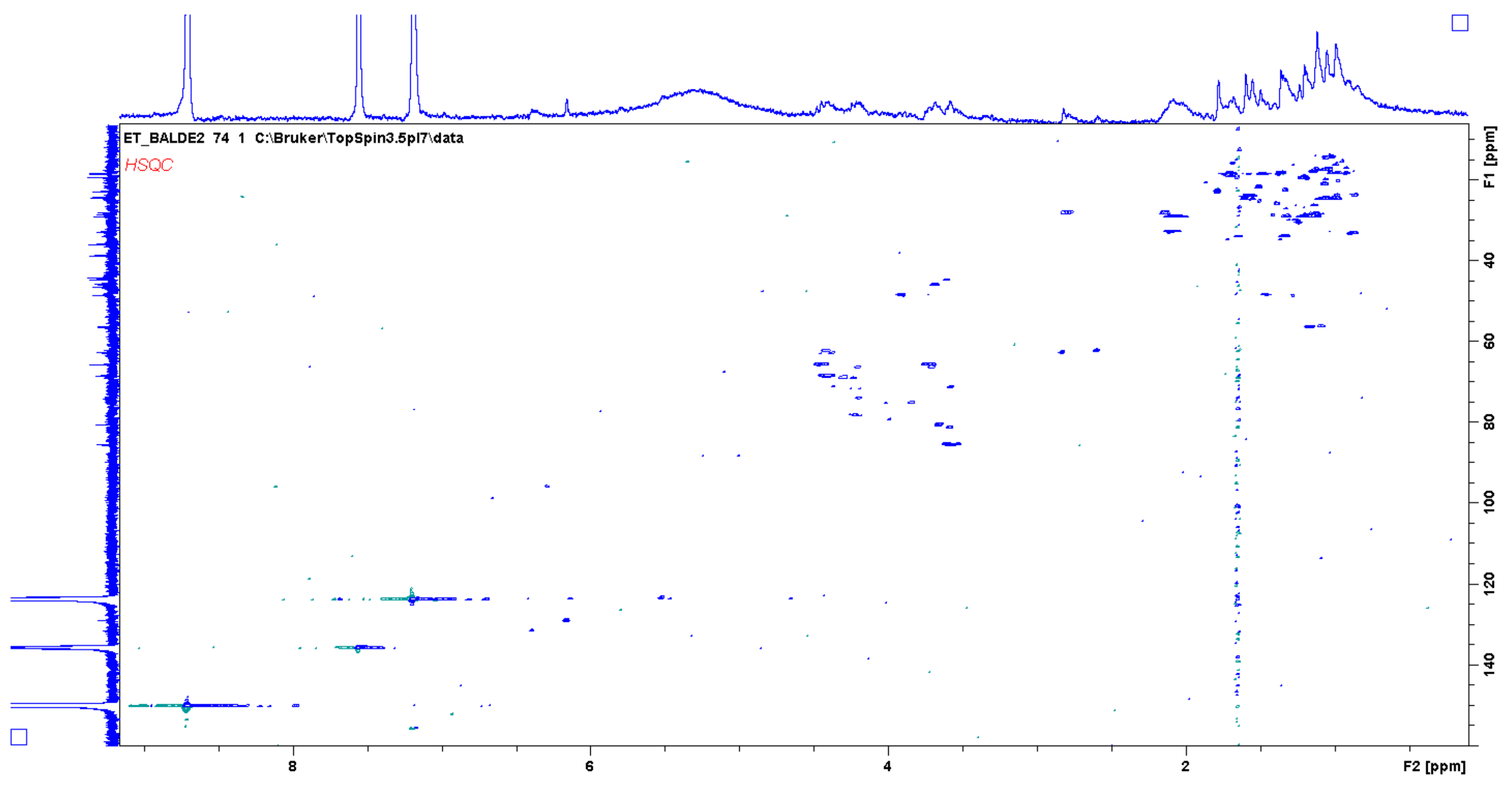

Figure S38. HSQC spectrum (pyridine- $d_{5}$ ) of compound (7) 


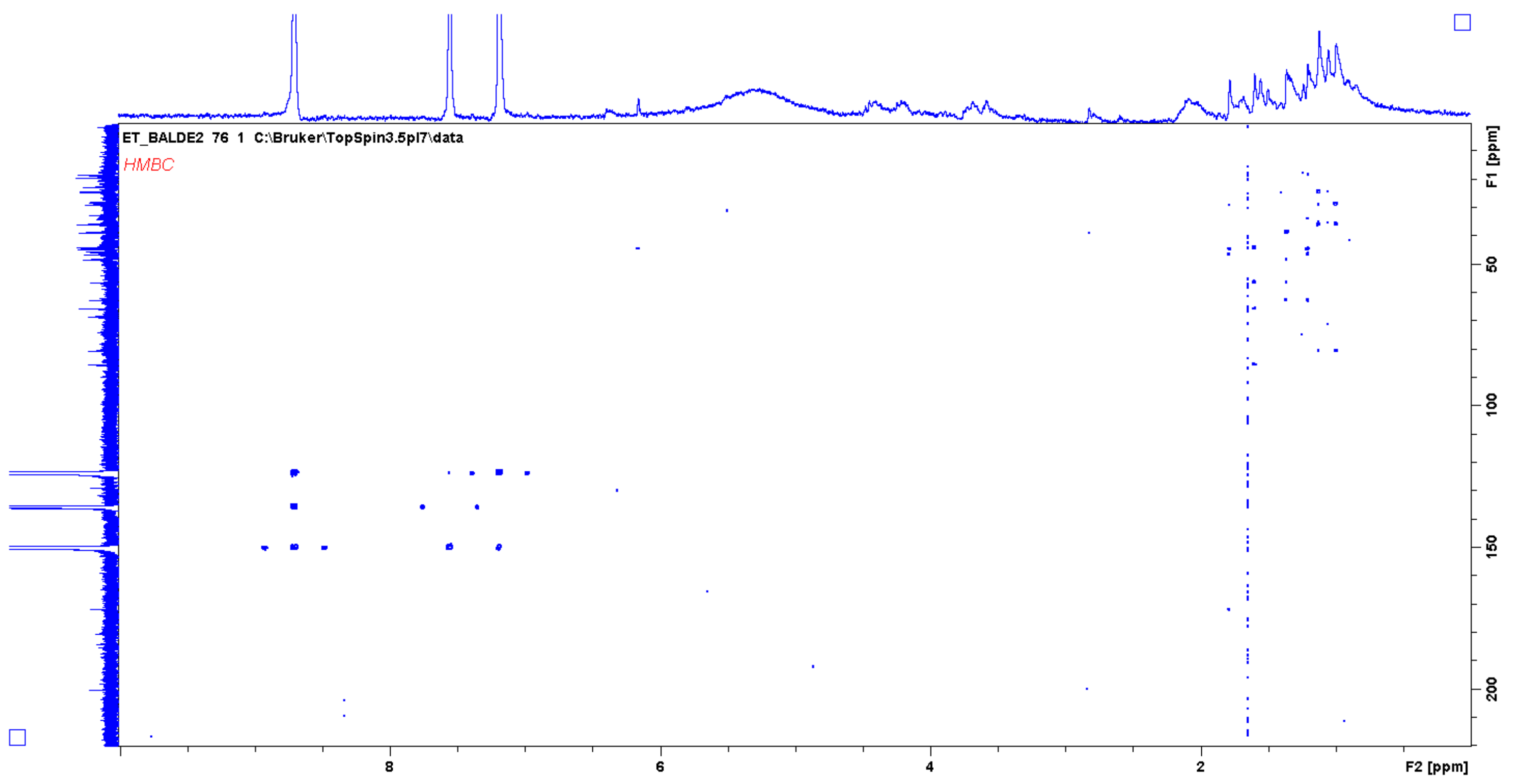

Figure S39. HMBC spectrum (pyridine- $d_{5}$ ) of compound (7) 


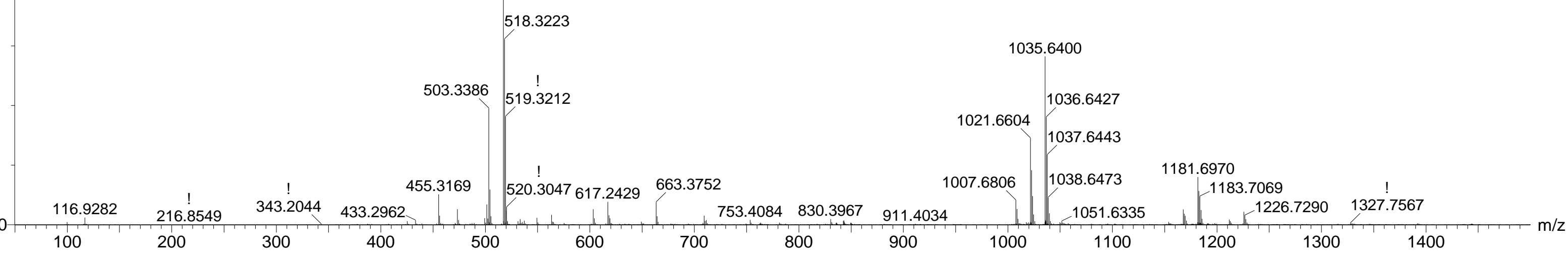

Figure S40. HR-ESI-MS spectrum of compound (7) 

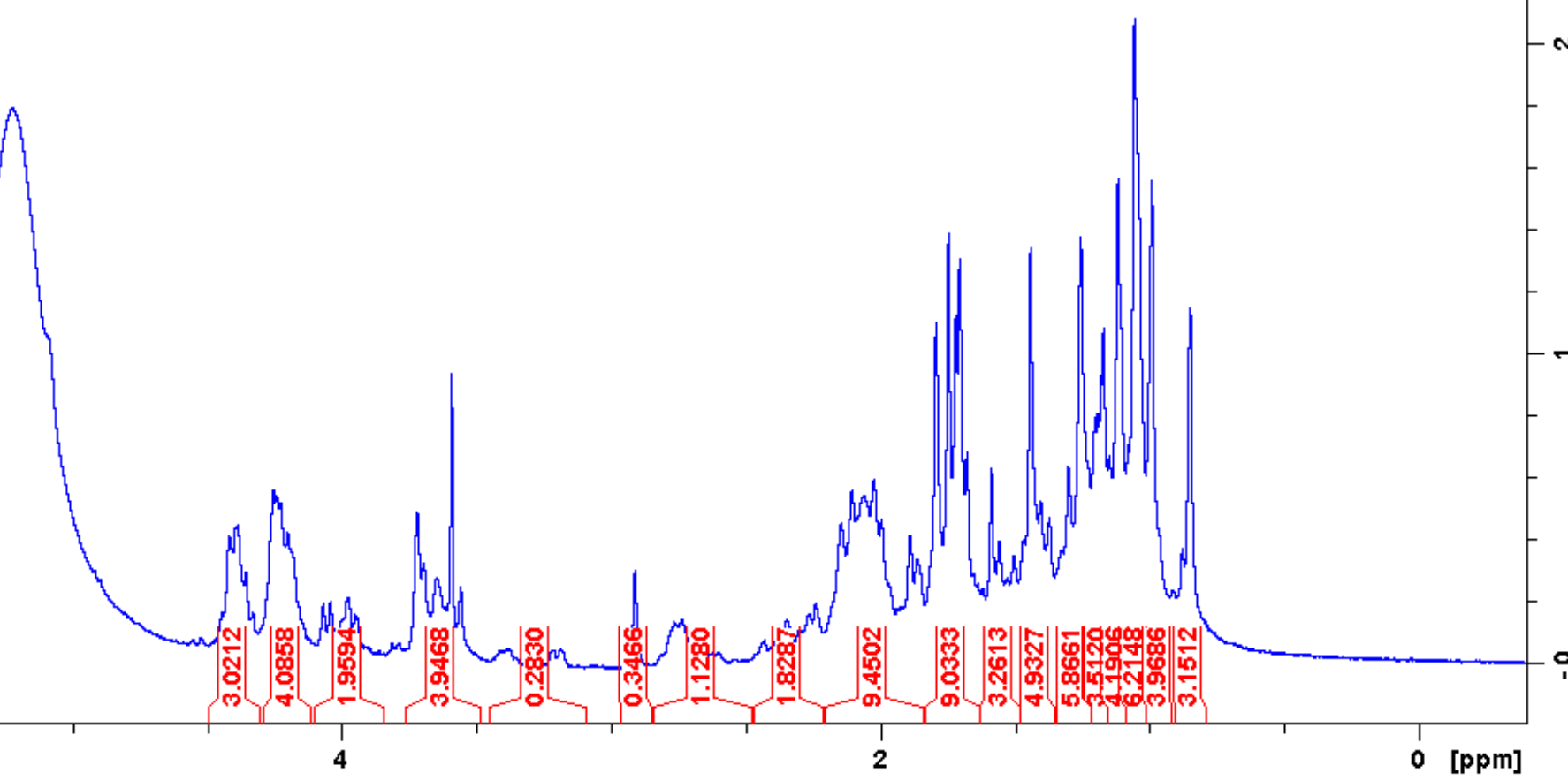

Figure S41. ${ }^{1} \mathrm{H}$ NMR spectrum (pyridine- $d_{5}$ ) of compound (8) 


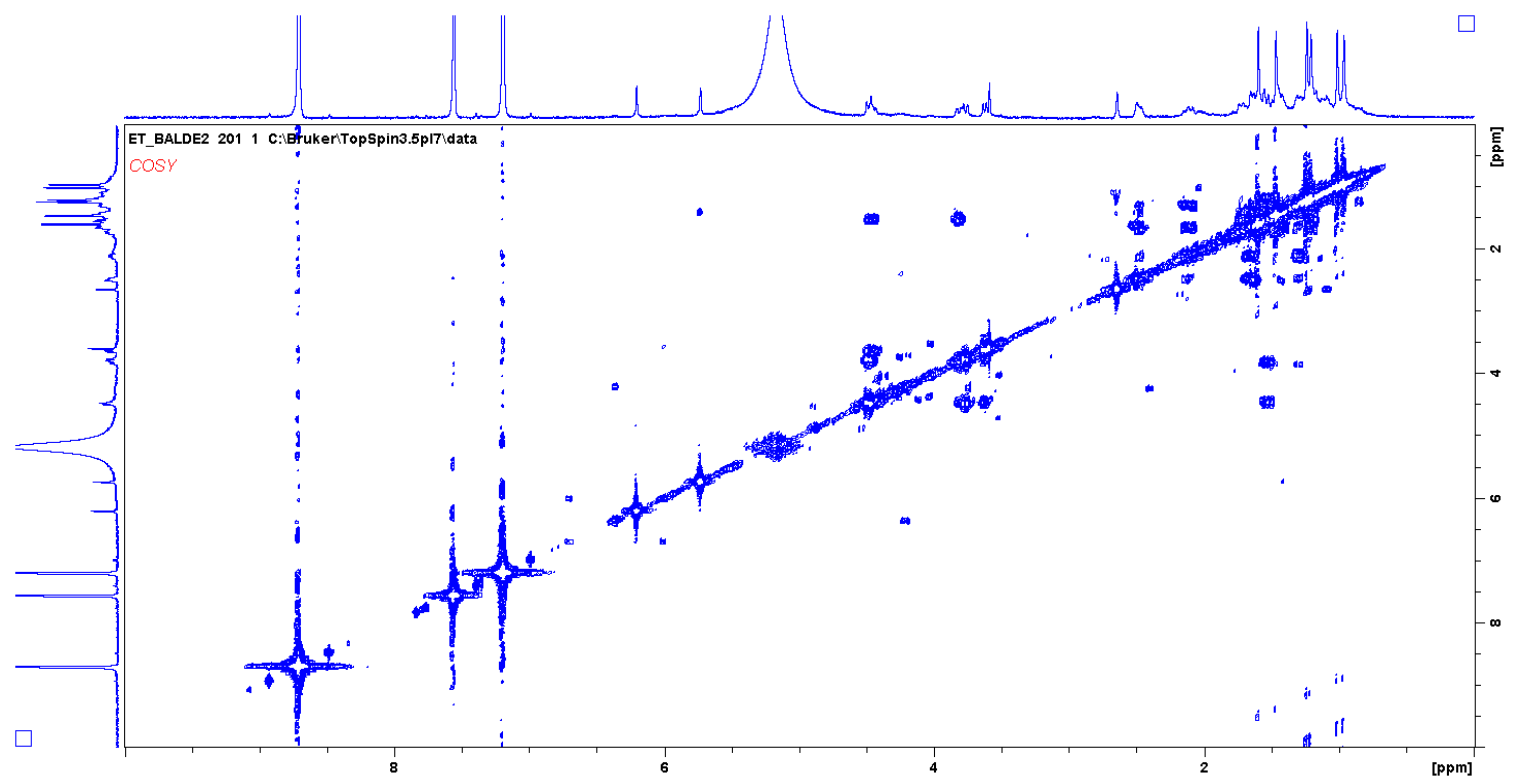

Figure S42. COSY spectrum (pyridine- $d_{5}$ ) of compound (8) 


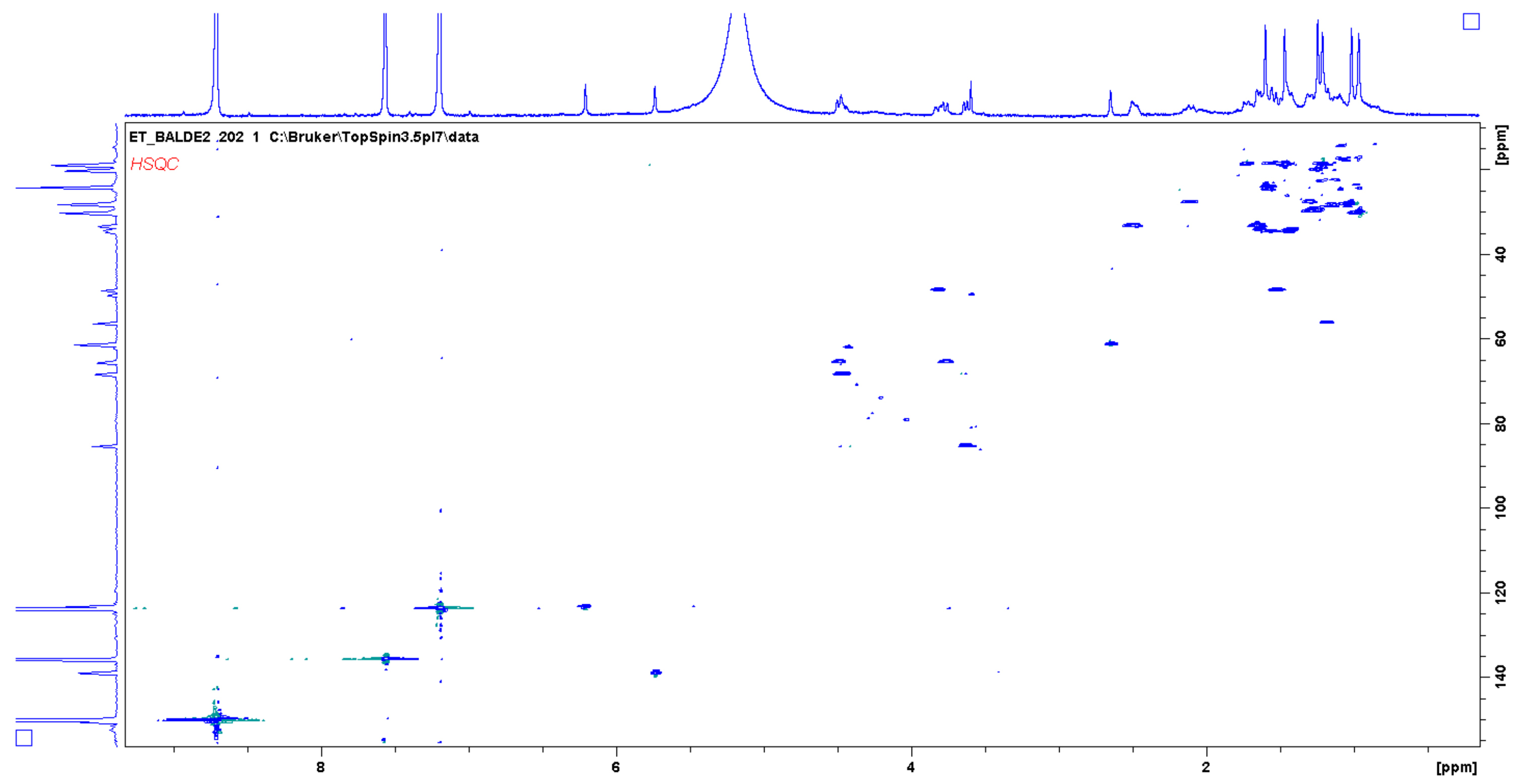

Figure S43. HSQC spectrum (pyridine- $d_{5}$ ) of compound (8) 


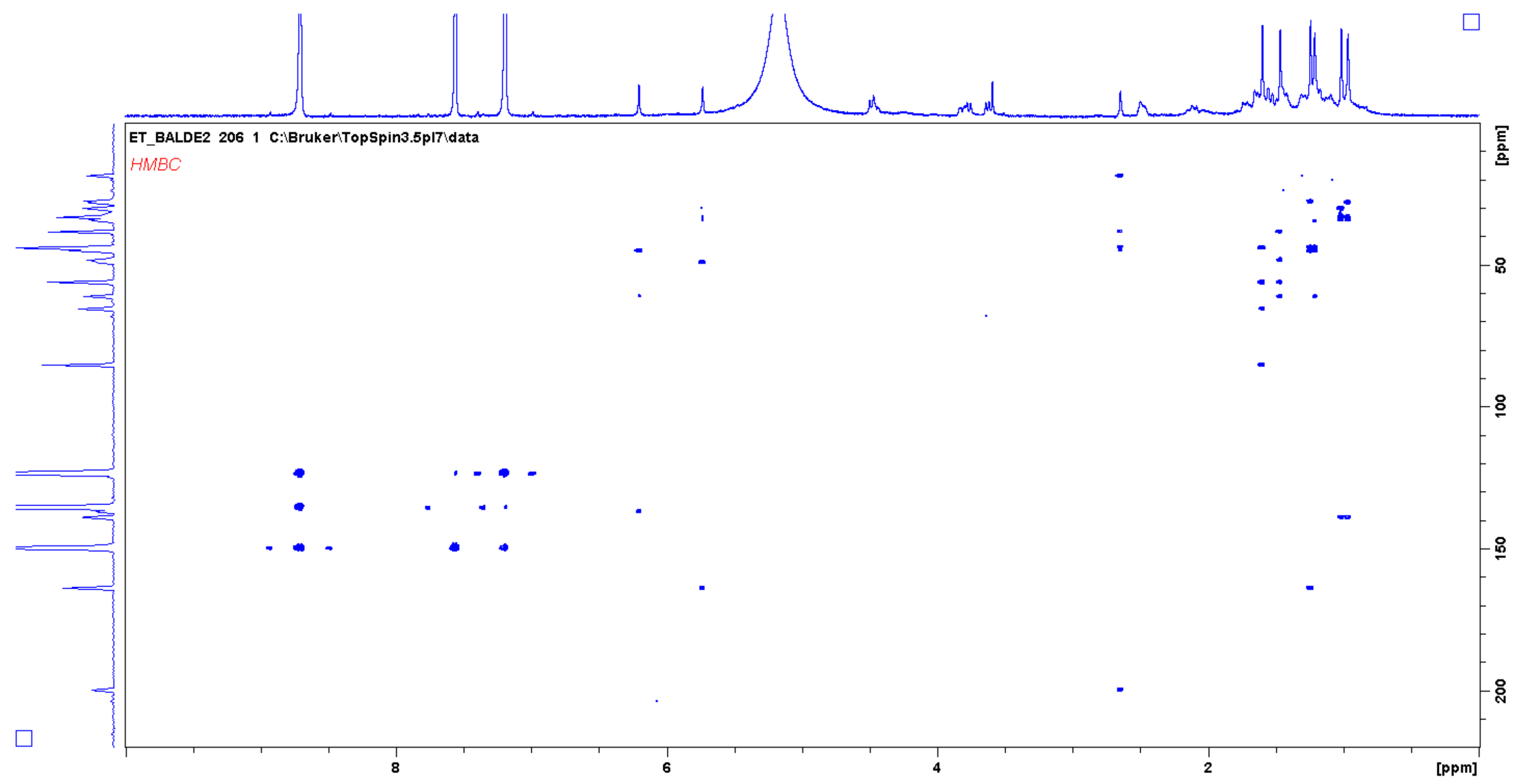

Figure S44. HMBC spectrum (pyridine- $d_{5}$ ) of compound (8) 


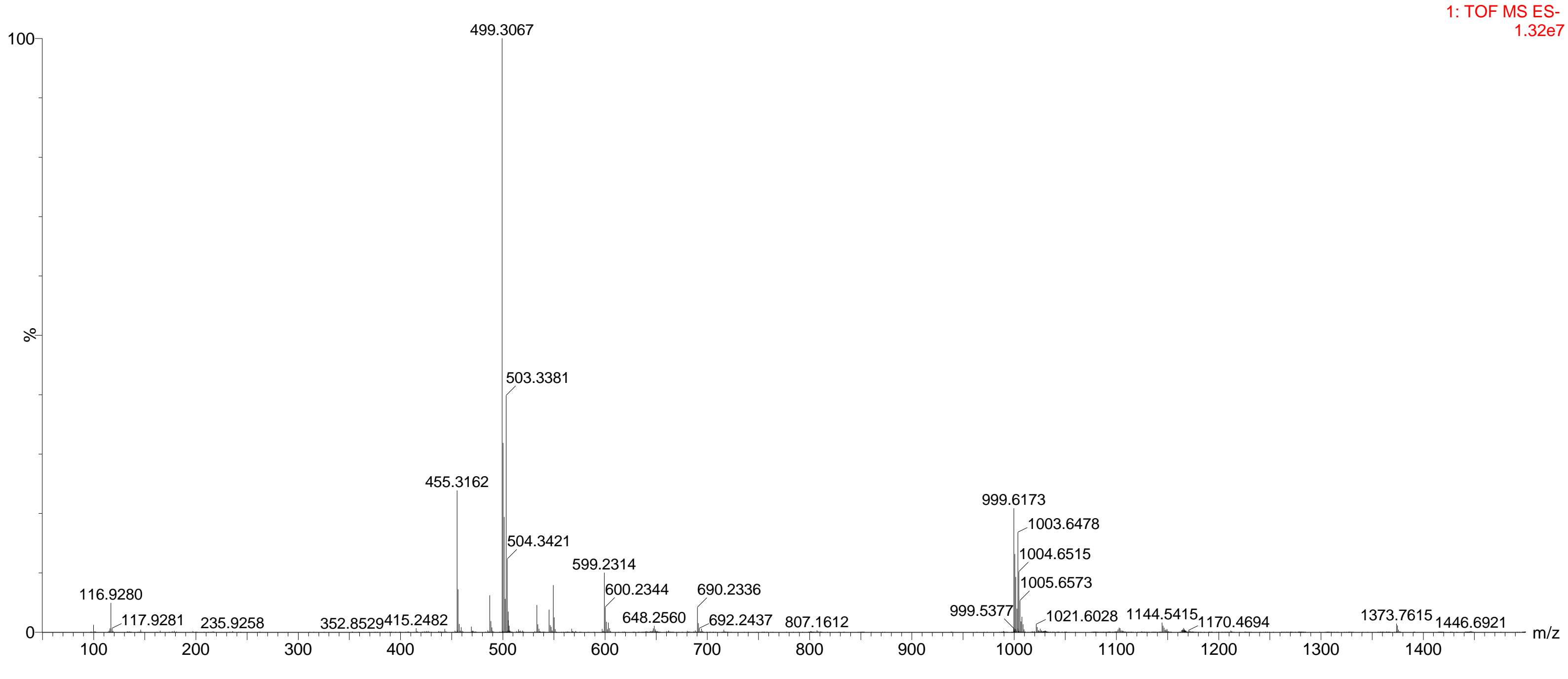

Figure S45. HR-ESI-MS spectrum of compound (8) 


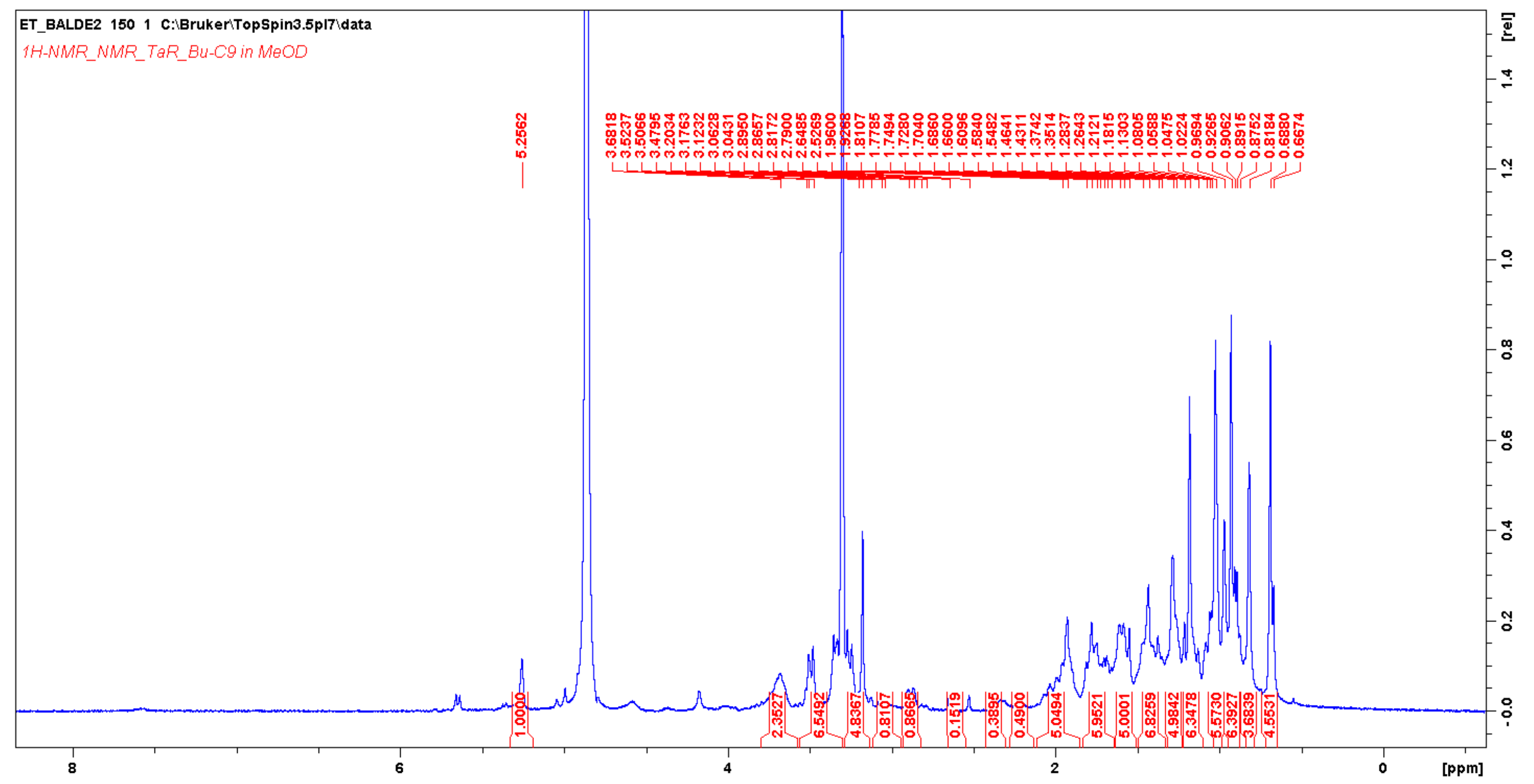

Figure S46. ${ }^{1} \mathrm{H}$ NMR spectrum (methanol- $d_{4}, 400 \mathrm{MHz}$ ) of compound (9) 


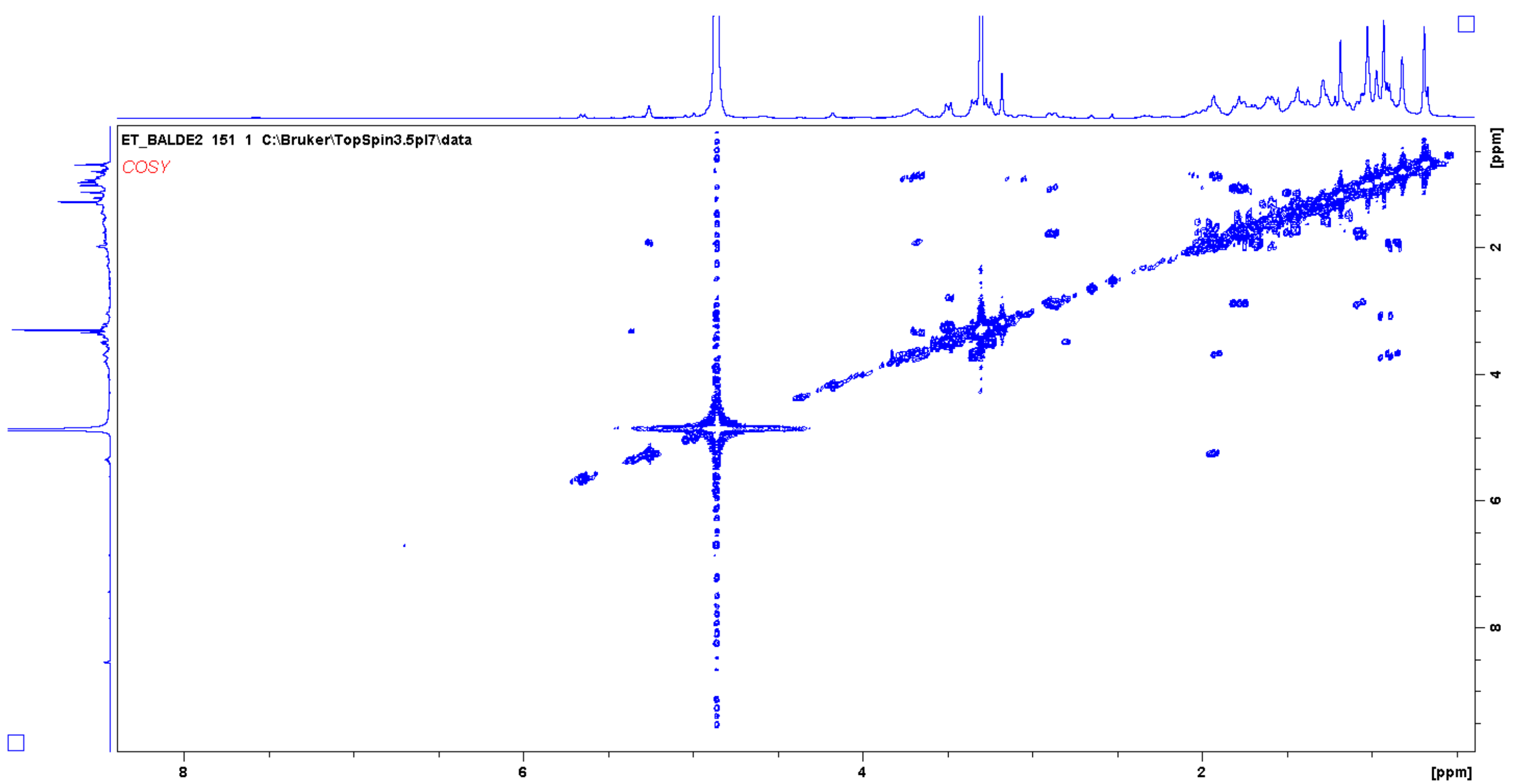

Figure S47. COSY spectrum (methanol- $d_{4}$ ) of compound (9) 


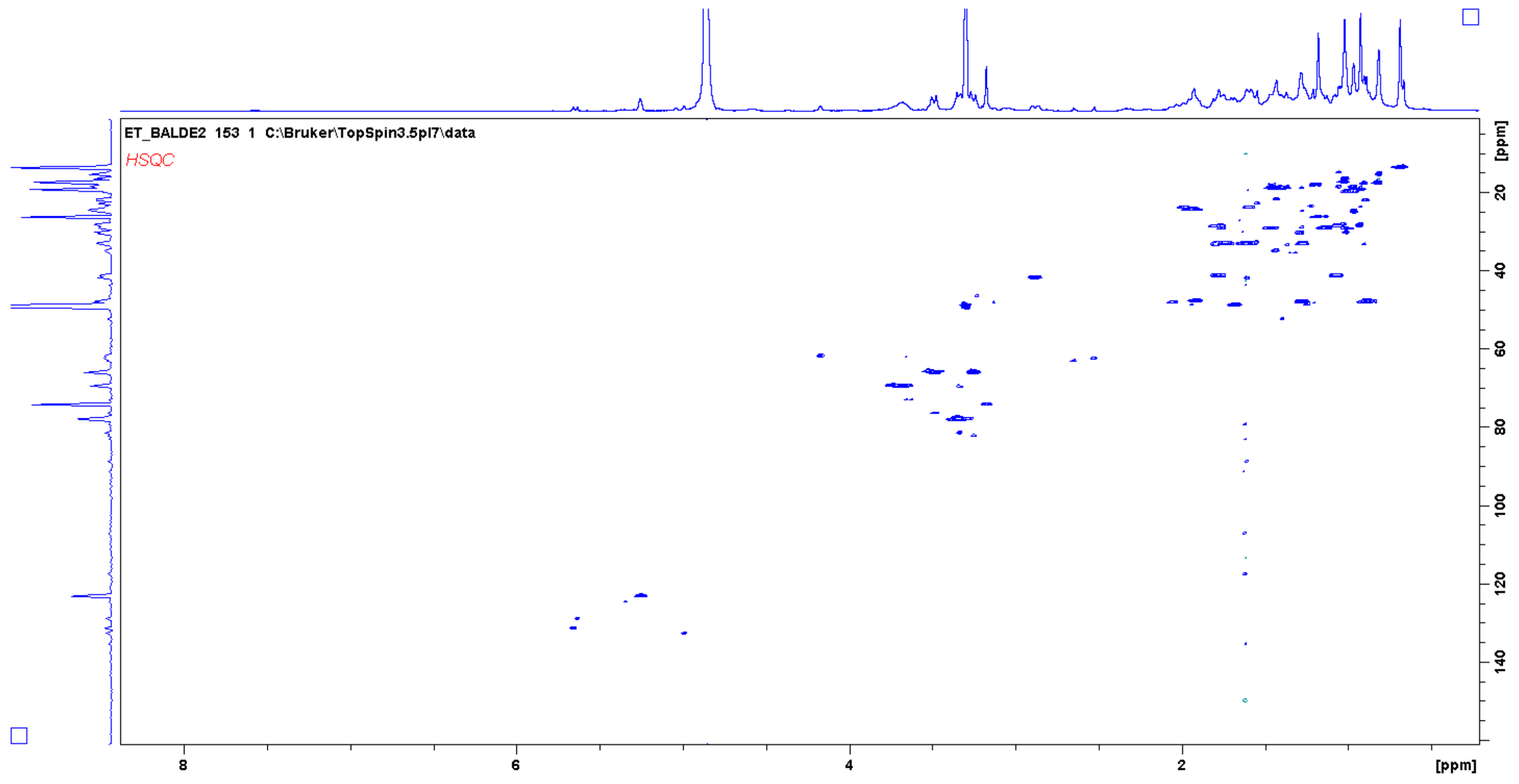

Figure S48. HSQC spectrum (methanol- $d_{4}$ ) of compound (9) 


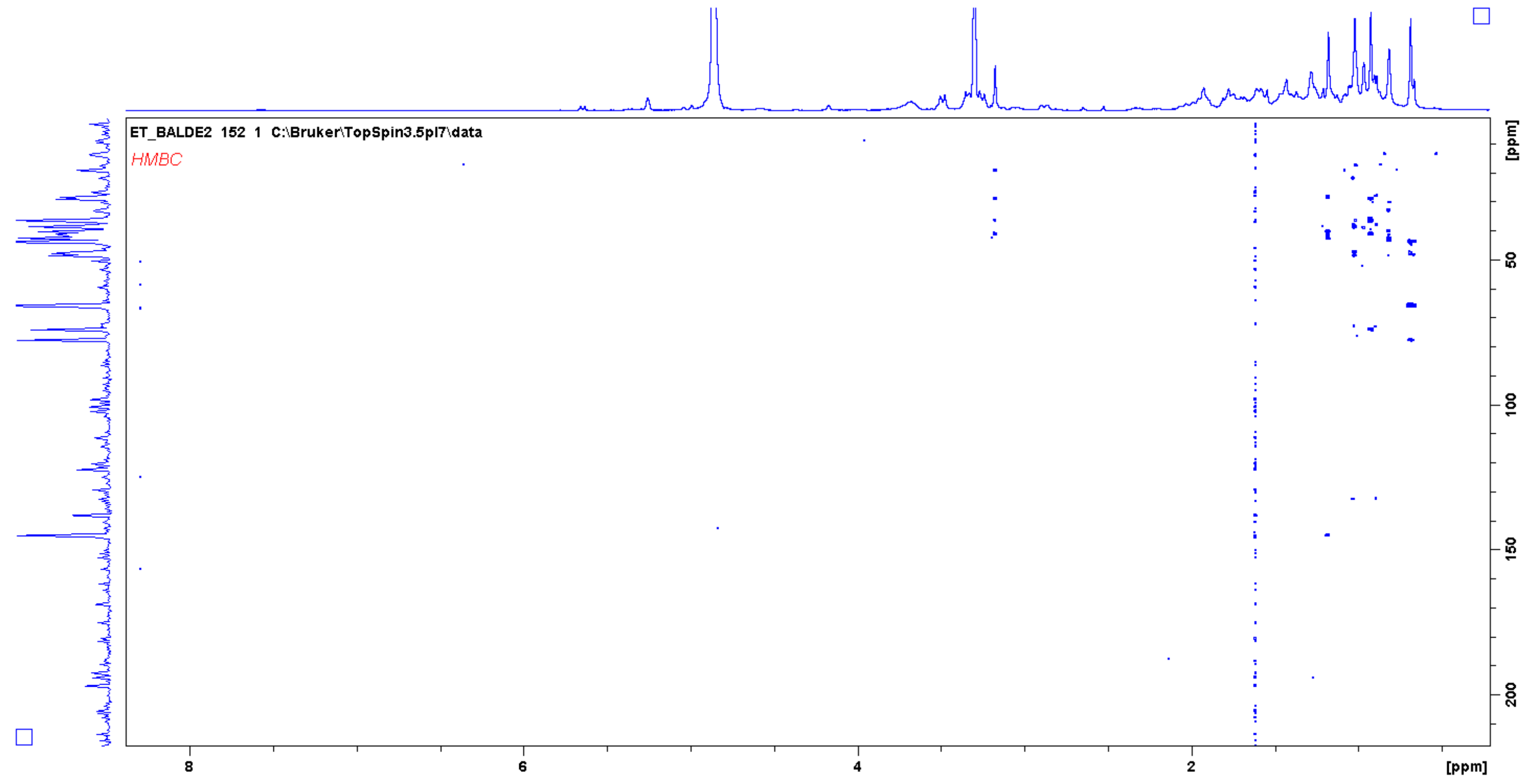

Figure S49. HMBC spectrum (methanol- $d_{4}$ ) of compound (9) 


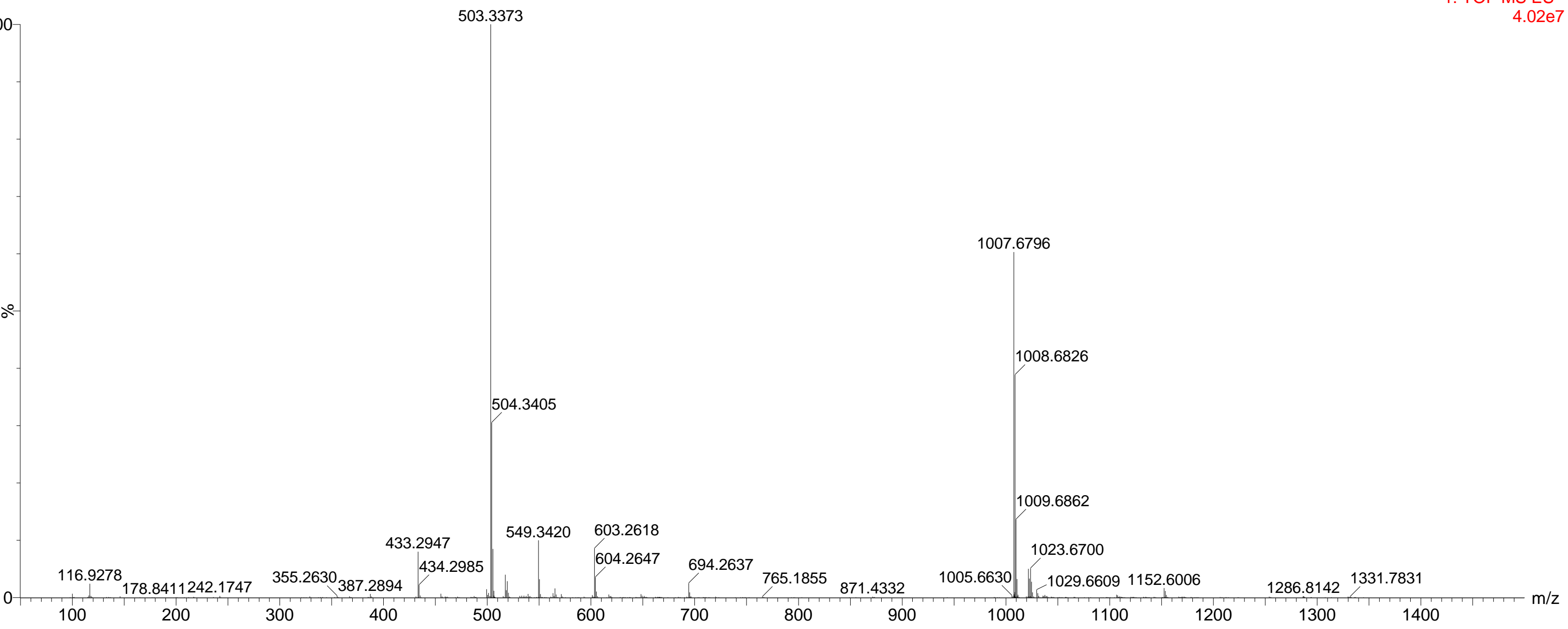

Figure S50. HR-ESI-MS spectrum of compound (9) 


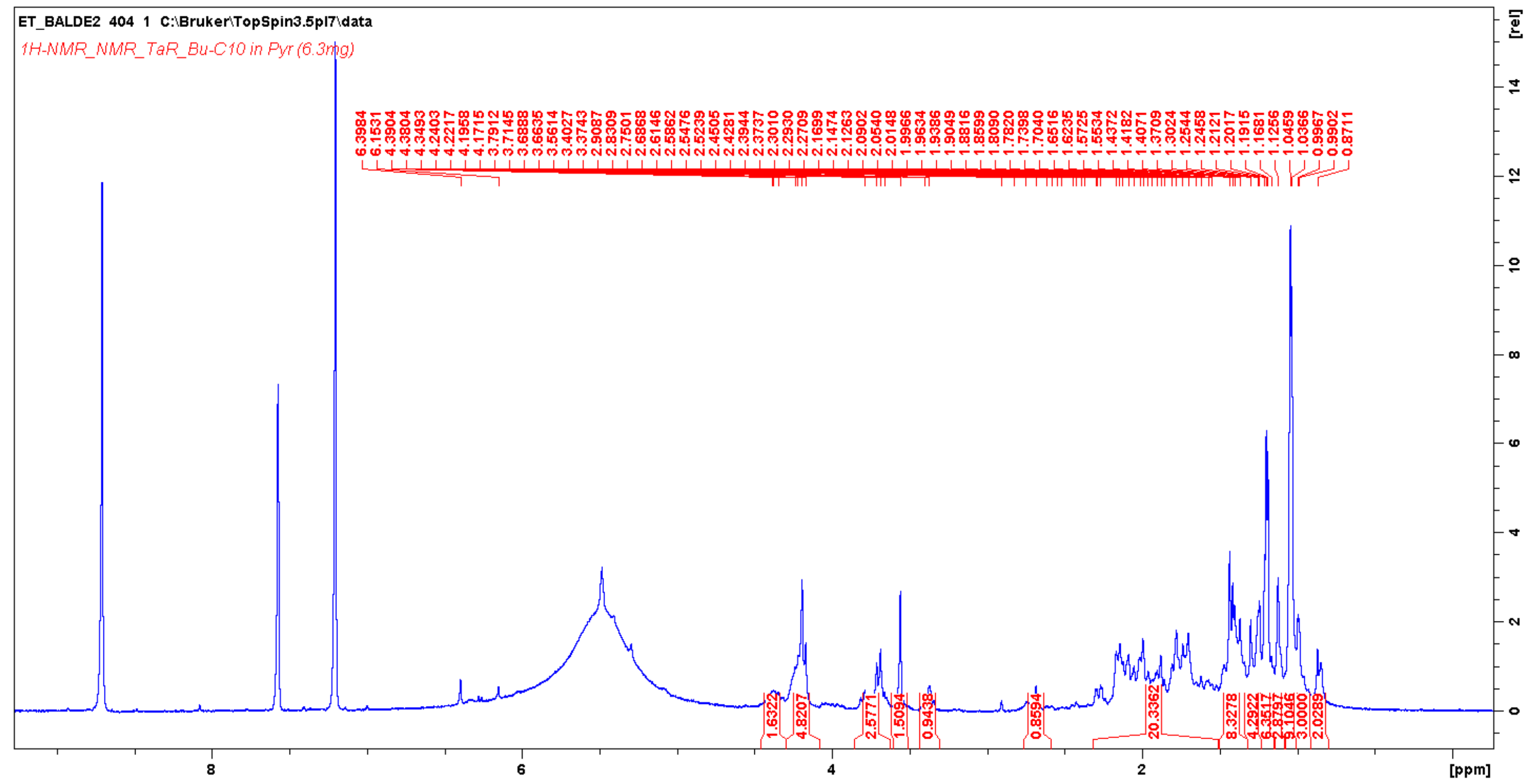

Figure S51. ${ }^{1} \mathrm{H}$ NMR spectrum (Pyridine- $d_{5}$ ) of compound (10) 


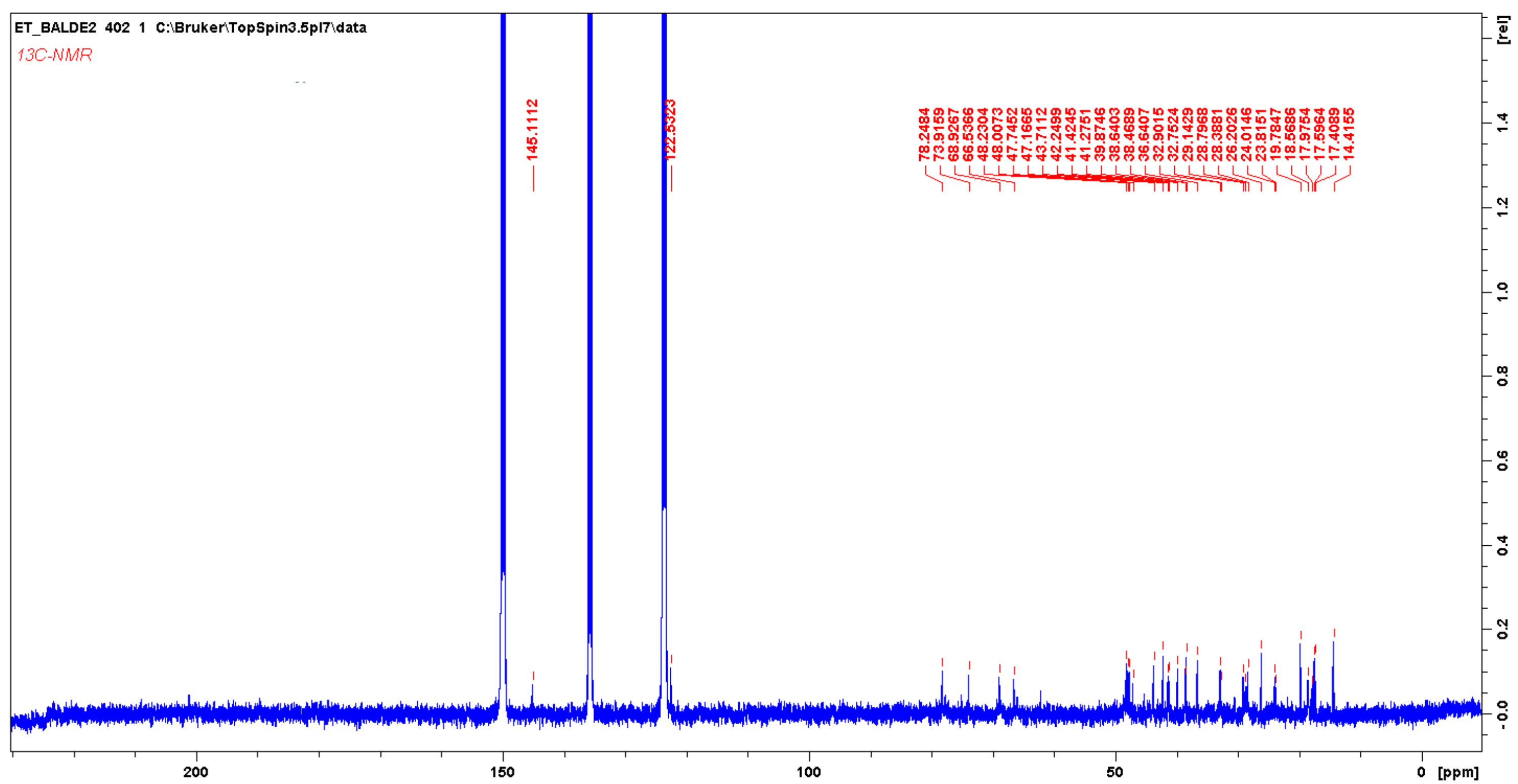

Figure S52. ${ }^{13} \mathrm{C}$ NMR spectrum (Pyridine- $d_{5}, 100 \mathrm{MHz}$ ) of compound (10) 


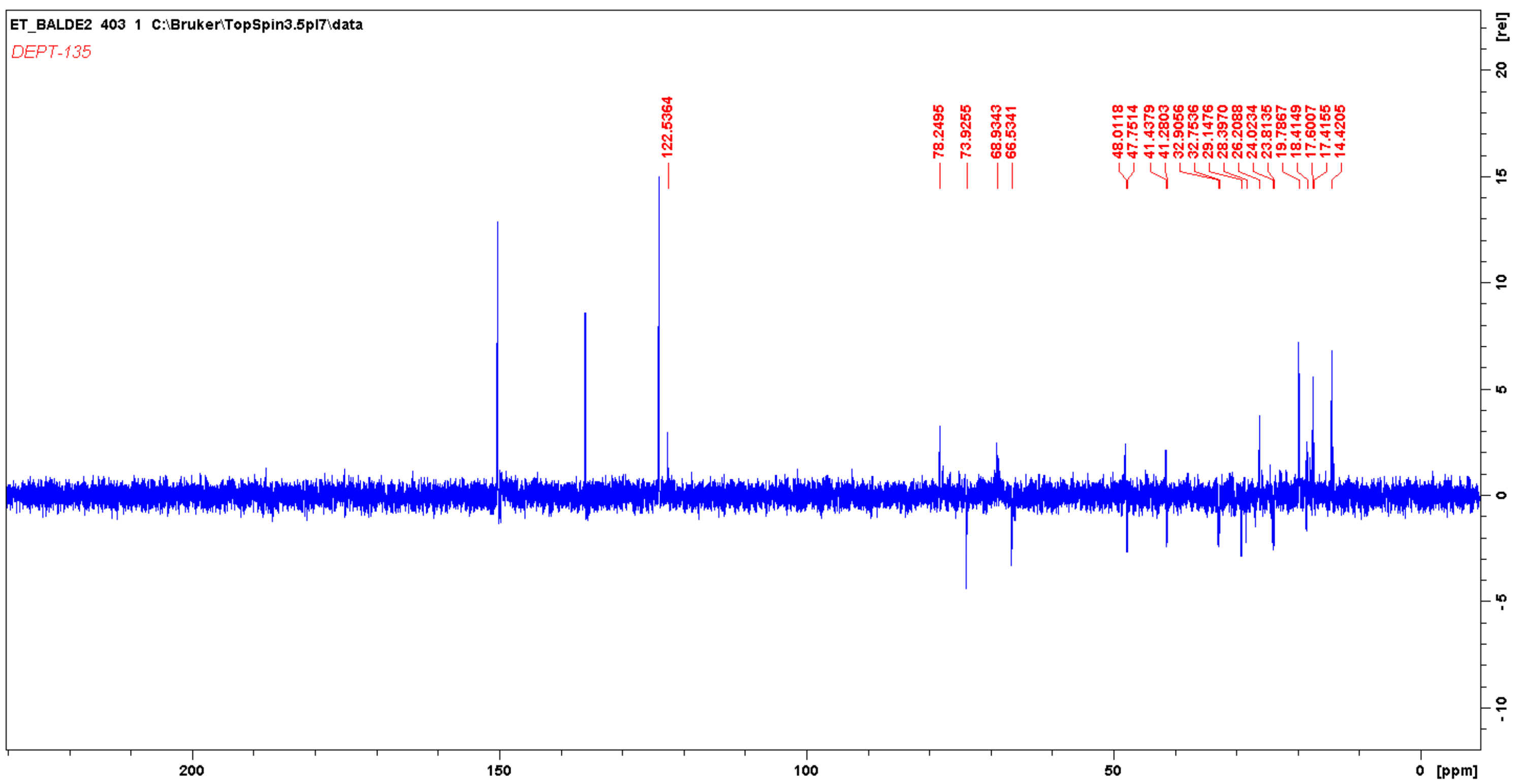

Figure S53. DEPT-135 NMR spectrum (Pyridine- $d_{5}, 100 \mathrm{MHz}$ ) of compound (10) 


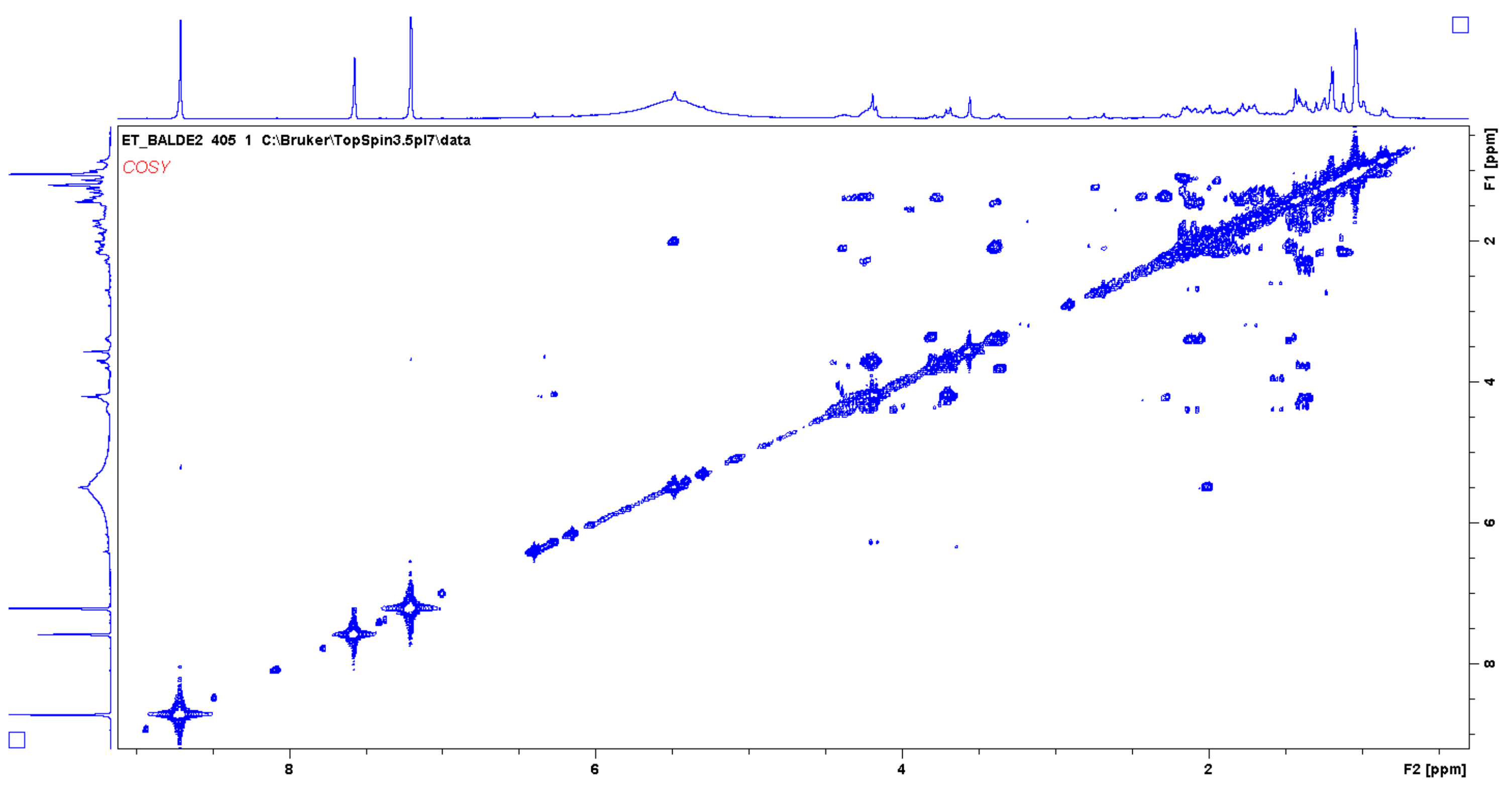

Figure S54. COSY spectrum (Pyridine- $d_{5}$ ) of compound (10) 


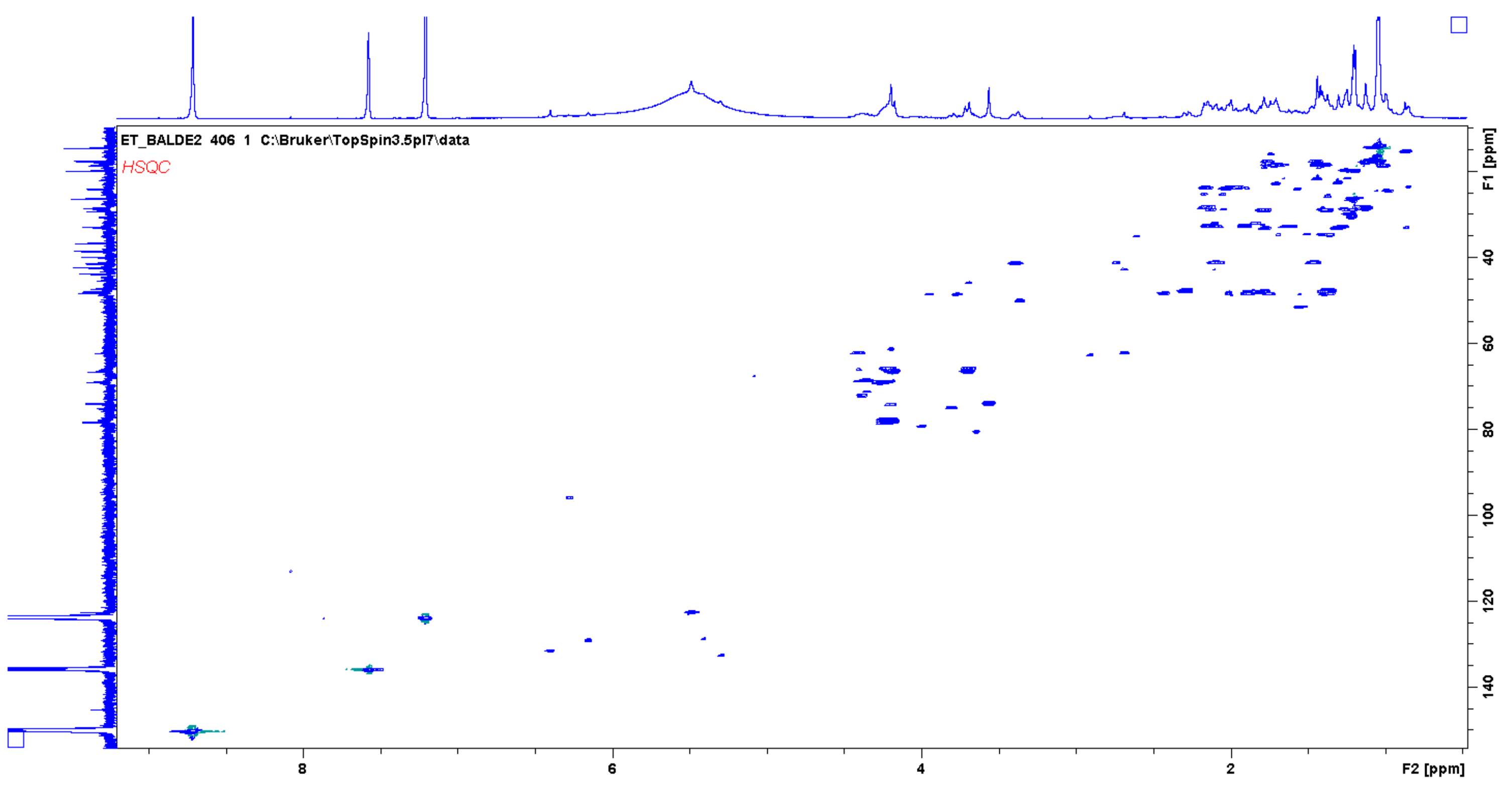

Figure S55. HSQC spectrum (methanol- $d_{4}$ ) of compound (10) 


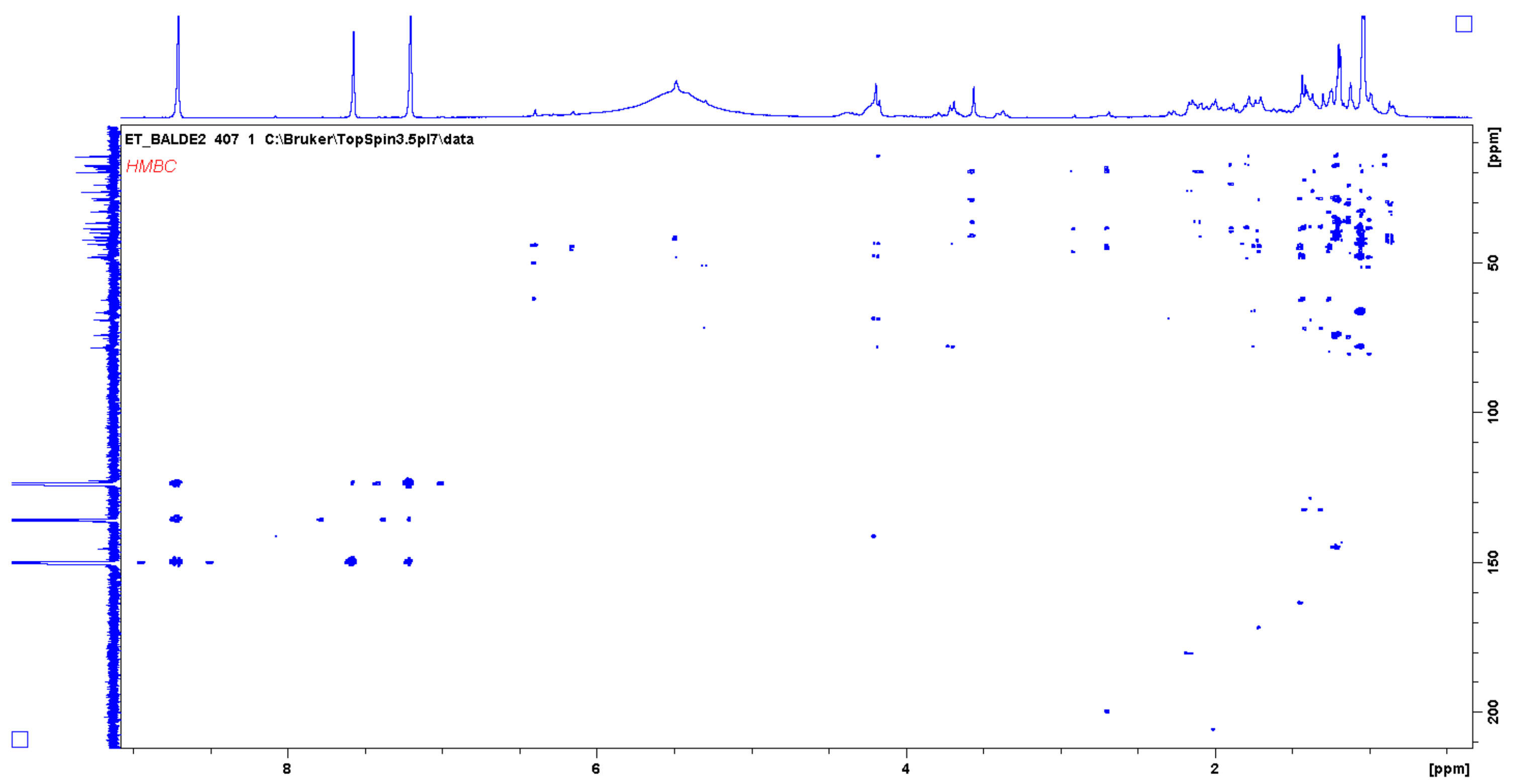

Figure S56. HMBC spectrum (methanol- $d_{4}$ ) of compound (10) 


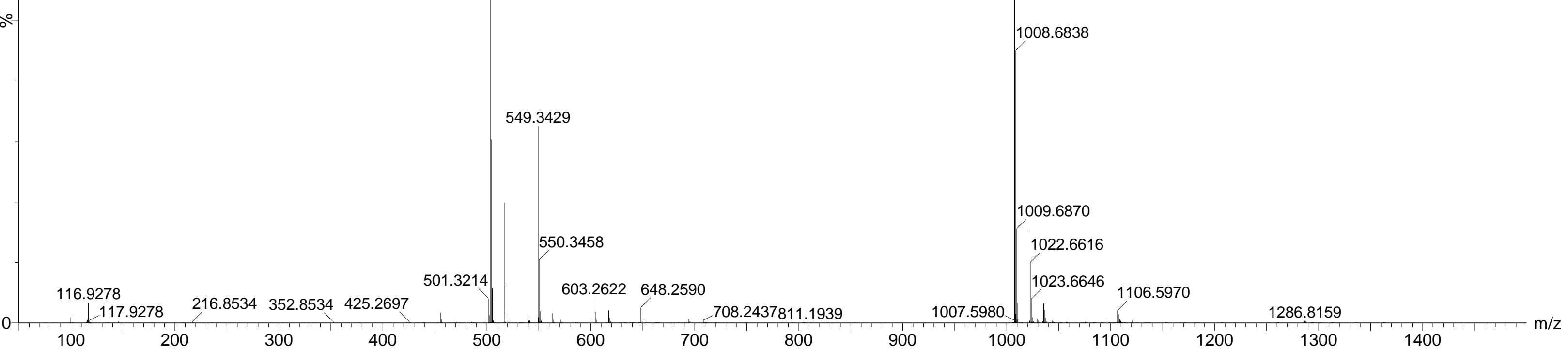

Figure S57. HR-ESI-MS spectrum of compound (10) 